\title{
REDUCTIVE COMPACT HOMOGENEOUS CR MANIFOLDS
}

\author{
ANDREA ALTOMANI, COSTANTINO MEDORI, AND MAURO NACINOVICH
}

\begin{abstract}
We consider a class of compact homogeneous $C R$ manifolds, that we call $n$-reductive, which includes the orbits of minimal dimension of a compact Lie group $\mathbf{K}_{0}$ in an algebraic homogeneous variety of its complexification $\mathbf{K}$. For these manifolds we define canonical equivariant fibrations onto complex flag manifolds. The simplest example is the Hopf fibration $S^{3} \rightarrow \mathbb{C P}^{1}$.

In general these fibrations are not $C R$ submersions, however they satisfy a weaker condition that we introduce here, namely they are $C R$-deployments.
\end{abstract}

\section{Contents}

1. Introduction

2. $\quad C R$ manifolds and $C R$ maps

3. Homogeneous $C R$ manifolds and their $C R$ algebras

4. Compact homogeneous $C R$ manifolds and n-reductiveness

5. A class of n-reductive compact homogeneous $C R$ manifolds

6. Parabolic regularization

7. The deployment theorem

8. $\quad$ Lifted $C R$ structures

9. A generalization

10. Some examples

References

\section{INTRODUCTION}

In this article we focus our attention on a class of compact homogeneous $C R$ manifolds. Our objects will turn out to be minimal orbits of a compact Lie group in their equivariant canonical $C R$-embedding (see $\$ 4.6$. We call them here $\mathrm{n}$ reductive (Definition 4.3), because the complex Lie algebra describing their homogeneous $C R$ structure is the direct sum of a nilpotent ideal and of the complexification of their isotropy. Compact homogeneous $C R$ manifolds and associated canonical bundles were also considered in [1, 2, 11]. We shall also consider various canonical fibrations. Let us consider a simple example.

Let $Q$ be the non degenerate Hermitian quadric in $\mathbb{C P}^{n}$, with index $q \leq\left[\frac{n}{2}\right]$. It is the minimal orbit of the natural action of $\mathbf{S U}_{q+1, n-q}$ in $\mathbb{C P}^{n}$. The choice of two $Q$-polar subspaces $S_{1}, S_{2}$ of $\mathbb{C P}^{n}$, of dimension $q$ and $n-q-1$, respectively,

Date: November 15, 2018.

2000 Mathematics Subject Classification. Primary: 32V05 Secondary: 32L05, 53C30.

Key words and phrases. Compact homogeneous $C R$ manifold, $C R$ algebra, equivariant fibration. 
that do not intersect $Q$, is equivalent to that of a maximal compact subgroup $\mathbf{K}_{0} \simeq$ $\mathbf{S}\left(\mathbf{U}_{q+1} \times \mathbf{U}_{n-q}\right)$ of $\mathbf{S} \mathbf{U}_{q+1, n-q}$. This choice defines a map $\pi: Q \rightarrow S_{1} \times S_{2}$ by

$$
\begin{gathered}
Q \ni p \longrightarrow \pi(p)=\left(\pi_{1}(p), \pi_{2}(p)\right) \in S_{1} \times S_{2}, \\
\text { with }\left\{\pi_{1}(p)\right\}=S_{1} \cap p S_{2},\left\{\pi_{2}(x)\right\}=S_{2} \cap p S_{1},
\end{gathered}
$$

which presents $Q$ as a circle bundle over $\mathbb{C P}^{q} \times \mathbb{C P}^{n-q-1}$, and reduces, for $q=0$, to the classical Hopf fibration of the odd dimensional sphere. The projection $\pi$ is a $C R$-submersion, being the restriction of a holomorphic submersion from $V=\mathbb{C P}^{n} \backslash$ $\left(S_{1} \cup S_{2}\right)$, with fibers biholomorphic to $\mathbb{C}^{*}$. We note that $V$ is a homogeneous space of the complexification $\mathbf{K} \simeq \mathbf{S}\left(\mathbf{G L}_{q+1}(\mathbb{C}) \times \mathbf{G L}_{n-q}\right)$ of $\mathbf{K}_{0}$ and that the immersion $Q \hookrightarrow V$ is induced by the inclusion $\mathbf{K}_{0} \subset \mathbf{K}$.

We shall generalize this construction to general $\mathfrak{n}$-reductive homogeneous compact $C R$ manifolds. This class contains the minimal orbits of real forms in complex flag manifolds (see [3]), and, more in general, the compact homogeneous $C R$ manifold arising as intersections of general orbits with their Matsuki dual (see [15, 5]).

Our generalization of the Hopf fibration will yield $C R$ maps which are smooth submersions, but which may fail, in general, to be $C R$-submersions. They satisfy, however, a weaker requirement, which lead us to the notions of $C R$-spread and $C R$-deployment. A $C R$ map $\pi: M \rightarrow N$ is a $C R$-spread if $N$ has the minimal $C R$ structure for which $\pi$ is a $C R$ map, and a $C R$-deployment if, moreover, $d \pi$ is injective on the analytic tangent bundle. We prove that an $\mathfrak{n}$-reductive $M$ has a $C R$ deployment over a complex flag manifold by a map that restricts a holomorphic fibrations with Stein fibers (see Theorems7.8, 7.9). This notion has also a counterpart for foliated complex manifolds, connected to the Segre varieties associated to a $C R$ manifold (see Theorem7.13).

Let $\pi: M \rightarrow N$ be a smooth submersion of an $m$-dimensional smooth manifold $M$ onto an $n$-dimensional complex manifold $N$. An Ehresmann connection $\mathcal{H}$ on $M \stackrel{\pi}{\longrightarrow} N$ uniquely defines an almost $C R$ structure on $M$ for which the analytic tangent space is the horizontal distribution and $\pi$ a $C R$-submersion. The $C R$ structure on the nondegenerate Hermitian quadric $Q$ is obtained from its Hopf fibration $\pi$ if we define $\mathcal{H}$ to be the analytic tangent space to $Q$.

Definition 1.1. Let $M$ be a $C R$ manifold of $C R$ dimension $n$ and $C R$ codimension $k, N$ an $n$-dimensional complex manifold. If $\pi: M \rightarrow N$ a $C R$-submersion, for which the analytic tangent space $H M$ is the horizontal distribution of an Ehresmann connection, then we say that the $C R$ structure of $M$ lifts the complex structure of $N$.

In general, an Ehresmann connection $\mathcal{H}$ on $M \stackrel{\pi}{\rightarrow} N$ needs to satisfy strong integrability conditions for the lifted structure to be $C R$. Our construction in $\$ 8$ shows that any n-reductive $C R$ structure on $M$ can be strengthened to the lift of a complex flag manifold. This also solves the problem of determining all homogeneous nt-reductive $C R$ structures on a given smooth compact homogeneous manifold. Similar problems were considered for instance in [9, 4].

Let us briefly describe the contents of this paper. We collected in $\$ 2$ and $\$ 3$ the preliminary notions on $C R$ manifolds and homogeneous $C R$ manifolds. In $\$ 4$ we consider specifically those which are homogeneous for the action of a compact Lie group, and introduce the notion of n-reductive. In $\$ 5$ we show that the intersection of the orbits of the real forms in complex flag manifolds with their Matsuki duals are n-reductive. In $\$ 6$ we recall a classical construction of Chevalley (see e.g. [7]) 
which is preliminary to the construction of the deployments and the lifts of $\$ 7$ and $\$ 8$. In $\$ 9$ we make some remarks for the case of compact homogeneous $C R$ manifolds which are not $\mathrm{n}$-reductive. In the final $\$ 10$ we discuss some examples.

\section{2. $C R$ MANIFOLDS AND $C R$ MAPS}

Let $M$ be a smooth manifold of real dimension $m$, and $n_{\mu}, k_{\mu} \geq 0$ two integers with $2 n_{\mu}+k_{\mu}=m$. A CR structure $\mu$ of type $\left(n_{\mu}, k_{\mu}\right)$ on $M$ is the datum of a rank $n_{\mu}$ smooth complex vector subbundle $T^{0,1} M$ of its complexified tangent bundle $\mathbb{C} T M$, with

$$
T^{0,1} M \cap \overline{T^{0,1} M}=\underline{0}, \quad\left[\Gamma\left(M, T^{0,1} M\right), \Gamma\left(M, T^{0,1} M\right)\right] \subset \Gamma\left(M, T^{0,1} M\right) .
$$

The integer $n_{\mu}$ is the $C R$-dimension, and $k_{\mu}=\operatorname{dim}_{\mathbb{R}} M-2 n_{\mu}$ the $C R$-codimension of $\mu=\left(M, T^{0,1} M\right)$. If $n_{\mu}=0$, we say that $\mu$ is totally real; if $k_{\mu}=0, \mu$ is a complex structure on $M$, in view of the Newlander-Nirenberg theorem.

Let $\mu=\left(M, T^{1,0} M\right)$ and $v=\left(N, T^{1,0} N\right)$ be two $C R$ manifolds.

A smooth map $\phi: M \rightarrow N$ is $C R$ if $d \phi\left(T^{0,1} M\right) \subset T^{0,1} N$. A $C R$-immersion (CR-embedding) is a smooth immersion (embedding) $\phi: M \rightarrow N$ such that

$$
d \phi\left(T^{1,0} M\right)=T^{1,0} N \cap d \phi\left(T^{\mathbb{C}} M\right) .
$$

A $C R$-immersion is called generic if, moreover, $d \phi\left(\mathbb{C} T_{p} M\right)+T_{\phi(p)}^{0,1} N=T_{\phi(p)}^{\mathbb{C}} N$ for all $p \in M$. This is the case when $n_{\mu}+k_{\mu}=n_{v}+k_{v}$.

A smooth submanifold $M$ of a $C R$ manifold $N$ is a $C R$-submanifold if $T^{1,0} M=$ $T^{\mathbb{C}} M \cap T^{1,0} N$ defines a $C R$ structure on $M$, i.e. if $T^{1,0} M$ is a complex distribution of constant rank on $M$.

A $C R$-submersion, or $C R$-bundle is a smooth submersion $\pi: M \rightarrow N$ such that $d \pi\left(T_{p}^{0,1} M\right)=T_{\pi(p)}^{0,1} N$ for all $p \in M$. In this case the fibers are $C R$-submanifolds of $M$. They are totally real if and only if $M$ and $N$ have the same $C R$-dimension, and totally complex if and only if $M$ and $N$ have the same $C R$-codimension.

A $C R$-diffeomorphism is a smooth diffeomorphism $\phi: M \rightarrow N$ which is at the same time a $C R$-immersion and a $C R$-submersion.

We recall the concept of strengthening a $C R$ structure (see [5, Def. 5.8]).

Definition 2.1. Let $M$ be a smooth manifold and $\mu_{1}, \mu_{2}$ two $C R$ structures on $M$. If the identity map from $\mu_{1}$ to $\mu_{2}$ is $C R$, we say that $\mu_{2}$ is a strengthening of $\mu_{1}$ or, equivalently, that $\mu_{1}$ is a weakening of $\mu_{2}$.

Let $M$ be a smooth manifold and denote by $\Omega_{M}$ the sheaf of germs of complex valued smooth exterior forms on $M$ and by $\mathfrak{X}_{M}^{\mathbb{C}}$ the sheaf of germs of complex valued smooth vector fields on $M$.

Definition 2.2 (ideal sheaf and characteristic distribution). The ideal sheaf $\mathscr{I}_{\mu}$ of $\mu=\left(M, T^{0,1} M\right)$ is the subsheaf of $\Omega_{M}$ consisting of the germs of forms of positive degree that vanish on $T^{0,1} M$. It is graded and $d$-closed. The characteristic distribution $\mathscr{Z}_{\mu}$ of $\mu$ is the sheaf of germs of smooth sections of $T^{0,1} M$.

If $\mu_{1}, \mu_{2}$ are $C R$ structures on the same smooth manifold $M$, necessary and sufficient conditions for $\mu_{2}$ to be a strengthening of $\mu_{1}$ is that $\mathscr{Z}_{\mu_{1}} \subset \mathscr{Z}_{\mu_{2}}$, or, equivalently, that $\mathscr{I}_{\mu_{2}} \subset \mathscr{I}_{\mu_{1}}$. 
Let $M$ be a smooth manifold, $v=\left(N, T^{0,1} N\right)$ a smooth $C R$ manifold and $\phi$ : $M \rightarrow N$ a smooth map. The pullback $\phi^{*} \mathscr{Z}_{v}$ of $\mathscr{Z}_{v}$ is the sheaf associated to the presheaf

$$
U \longrightarrow \phi^{*} \mathscr{Z}_{v}(U)=\left\{X \in \mathfrak{X}_{M}^{\mathbb{C}}(U) \mid d \phi\left(X_{p}\right) \in T^{0,1} N, \forall p \in U\right\} .
$$

Denote by $\phi^{*} \mathscr{I}_{v}$, the $C_{M}^{\infty}$-module generated by the pull-backs $\phi^{*} \omega$ of germs $\omega \in$ $\mathscr{I}_{v}$. The elements of $\phi^{*} \mathscr{I}_{v}$ vanish on $\phi^{*} \mathscr{Z}_{v}$ and the system $\phi^{*} \mathscr{Z}_{v}$ is formally integrable. Indeed, we have

$$
\left[\phi^{*} \mathscr{Z}_{v}, \phi^{*} \mathscr{Z}_{v}\right] \subset \phi^{*} \mathscr{Z}_{v}, \quad d\left(\phi^{*} \mathscr{I}_{v}\right) \subset \phi^{*} \mathscr{I}_{v} .
$$

One easily verifies the following

Lemma 2.3. Let $\mu=\left(M, T^{0,1} M\right)$ and $v=\left(N, T^{0,1} N\right)$ be CR manifolds, $\phi: M \rightarrow N$ a smooth map and $p_{0} \in M$. The following are equivalent

(1) $\phi$ is $C R$ on a neighborhood of $p_{0}$;

(2) $\phi^{*} \mathscr{I}_{v_{\phi\left(p_{0}\right)}} \subset \mathscr{I}_{\mu_{\left(p_{0}\right)}}$;

(3) $\mathscr{Z}_{\mu_{\left(p_{0}\right)}} \subset \phi^{*} \mathscr{Z}_{v_{\phi\left(p_{0}\right)}}$.

If $\phi: M \rightarrow N$ is a smooth map, we denote by $\mathscr{V}_{\phi}$ the sheaf of germs of $\phi-$ vertical smooth complex vector fields on $M$. This is the sheaf associated to the presheaf

$$
U \longrightarrow \mathscr{V}_{\phi}(U)=\left\{X \in \mathfrak{X}_{M}^{\mathbb{C}}(U) \mid d \phi\left(X_{p}\right)=0, \quad \forall p \in U\right\} .
$$

If $v=\left(N, T^{0,1} N\right)$ is a $C R$ structure on $N$, then $\mathscr{V}_{\phi} \subset \phi^{*} \mathscr{Z}_{v}$, and if $\phi$ is $C R$ for $\mu=\left(M, T^{0,1} M\right)$, then $\phi^{*} \mathscr{Z}_{v}$ contains the Lie subalgebra of $\mathfrak{X}_{M}^{\mathbb{C}}$ generated by $\mathscr{Z}_{\mu}+\mathscr{V}_{\phi}$.

It is therefore natural to introduce the following generalization of the notion of $C R$-submersion.

Definition 2.4 ( $C R$-spread, $C R$-deployment). Let $\mu=\left(M, T^{0,1} M\right), v=\left(N, T^{0,1} N\right)$ be $C R$ manifolds and $\phi: M \rightarrow N$ a smooth map.

We say that $\phi$ is a $C R$-spread at $p_{0} \in M$ if it is a smooth submersion at $p_{0}$ and, moreover

$$
\phi^{*} \mathscr{Z}_{v_{\left(p_{0}\right)}} \text { is the Lie algebra generated by } \mathscr{Z}_{\mu_{\left(p_{0}\right)}}+\mathscr{V}_{\phi\left(p_{0}\right)} \text {. }
$$

We say simply that $\phi$ is a CR-spread if $\phi: M \rightarrow N$ is a smooth submersion and

$$
\phi^{*} \mathscr{Z}_{\nu} \text { is the Lie algebra generated by } \mathscr{Z}_{\mu}+\mathscr{V}_{\phi} \text {. }
$$

We say that $\phi$ is a $C R$-deployment at $p_{0} \in M$ if it is a $C R$ spread at $p_{0}$ and moreover $d \phi: T_{p_{0}}^{0,1} M \rightarrow T_{q_{0}}^{0,1} N$ is injective.

We say simply that $\phi$ is a $C R$-deployment if it is a $C R$ deployment at all points.

Equivalently, a $C R$-deployment is a $C R$-spread with totally real fibers.

Roughly speaking, a $C R$ map $\phi: M \rightarrow N$ is a $C R$-spread when $\phi$ is a submersion and $N$ carries the weakest $C R$ structure for which $\phi$ is $C R$.

The $C R$ spread has a nice interpretation in terms of the sheaves

$$
\mathscr{O}_{\mu}=\left\{f \in C_{M}^{\infty} \mid d f \in \mathscr{I}_{\mu}\right\}, \quad \mathscr{O}_{v}=\left\{f \in C_{N}^{\infty} \mid d f \in \mathscr{I}_{v}\right\}
$$

of germs of smooth $C R$ functions on $M, N$.

Proposition 2.5. Let $M, N$ be $C R$ manifolds, with $C R$ structures $\mu, v$, respectively, and $\phi: M \rightarrow N$ a smooth submersion. 
(1) $\phi$ is a CR-spread at $p_{0} \in M$ if and only if

$$
\omega \in \mathscr{I}_{v_{\phi\left(p_{0}\right)}} \Longleftrightarrow \phi^{*} \omega \in \mathscr{I}_{\mu_{p_{0}}} .
$$

(2) In particular, if $\phi$ is a $C R$-spread at $p_{0} \in M$, then

$$
\mathscr{O}_{N_{\phi\left(p_{0}\right)}}=\left\{u \in \mathcal{C}_{N_{\phi\left(p_{0}\right)}}^{\infty} \mid\left[\phi^{*} u\right]_{\left(p_{0}\right)} \in \mathscr{O}_{M_{\left(p_{0}\right)}}\right\} .
$$

Condition (2.8) is also sufficient when $N$ is locally embeddable at $\phi\left(p_{0}\right)$.

We recall that $N$ is locally embeddable at $q_{0} \in N$ if the real parts of germs of smooth $C R$ functions provide coordinate patches at $q_{0}$. This is always the case when the $C R$ structure $v$ of $N$ is real-analytic.

\section{Homogeneous $C R$ MaNifoldS AND their $C R$ ALgebras}

3.1. Homogeneous $C R$ manifolds. Let $\mathbf{G}_{0}$ be a Lie group, $g_{0}$ its Lie algebra and $\mathfrak{g}=\mathbb{C} \otimes_{\mathbb{R}} \mathfrak{g}_{0}$ its complexification.

Definition 3.1. A $\mathbf{G}_{0}$-homogeneous $C R$ manifold is a $\mathbf{G}_{0}$-homogeneous space, endowed with a $\mathbf{G}_{0}$-equivariant $C R$ structure.

Let $M$ be a $\mathbf{G}_{0}$-homogeneous $C R$ manifold. Fix $p_{0} \in M$ and denote by $\mathbf{M}_{0}=$ $\left\{g \in \mathbf{G}_{0} \mid g \cdot p_{0}=p_{0}\right\}$ the isotropy subgroup of $M$ at $p_{0}$. Let $\pi: \mathbf{G}_{0} \rightarrow M \simeq$ $\mathbf{G}_{0} / \mathbf{M}_{0}$ be the canonical projection and $d \pi: \mathfrak{g} \rightarrow T_{p_{0}}^{\mathbb{C}} M$ the complexification of the differential of $\pi$ at the identity. We showed (see e.g. [5, §1]) that

$$
\mathfrak{q}=d \pi^{-1}\left(T_{p_{0}}^{0,1} M\right)
$$

is a complex Lie subalgebra of $\mathfrak{g}$, with $\mathfrak{q} \cap \mathfrak{g}_{0}=\mathfrak{m}_{0}=\operatorname{Lie}\left(\mathbf{M}_{0}\right)$.

Definition 3.2. The pair $\left(\mathfrak{g}_{0}, \mathfrak{q}\right)$ is the $C R$-algebra at $p_{0}$ of the $\mathbf{G}_{0}$-homogeneous $C R$ manifold $M$.

Clearly the datum of the $C R$ algebra at any single point of $M$ completely defines its $C R$ structure and we have:

Proposition 3.3. The $\mathbf{G}_{0}$-homogeneous $C R$ structures on $M$ are in one-to-one correspondence with the set of complex Lie subalgebras q of $\mathfrak{g}$ such that

(1) $\mathfrak{q} \cap \mathfrak{g}_{0}$ equals the Lie algebra $\mathrm{m}_{0}$ of $\mathbf{M}_{0}$,

(2) $\mathfrak{q}$ is $\operatorname{Ad}_{\mathfrak{g}}\left(\mathbf{M}_{0}\right)$-invariant.

This was our motivation to introduce and discuss $C R$ algebras in [18].

Let $M$ be a $\mathbf{G}_{0}$-homogeneous $C R$ manifold, with $C R$ algebra $\left(\mathfrak{g}_{0}, \mathfrak{q}\right)$ at $p_{0} \in M$. The complex valued $\mathbf{G}_{0}$-equivariant one-forms on $M$ are in one-to-one correspondence with the elements of the dual $\mathfrak{g}^{*}$ of $\mathfrak{g}$ which annihilate $\mathfrak{q} \cap \overline{\mathfrak{q}}$. For $\eta \in(\mathfrak{q} \cap \overline{\mathfrak{q}})^{0}$, we define $\eta_{p_{0}}^{*} \in{ }^{\mathbb{C}} T_{p_{0}}^{*} M$ by the commutative diagram

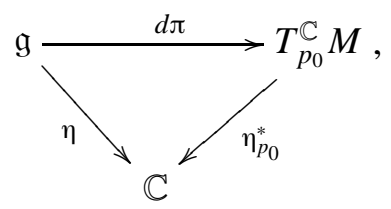

where $\pi: \mathbf{G}_{0} \ni g \rightarrow g \cdot p_{0} \in M$ is the natural projection. Then $\eta_{p_{0}}^{*}$ can be extended to a $\mathbf{G}_{0}$-equivariant one-form by setting $\eta_{g \cdot p_{0}}^{*}=g_{*} \eta_{p_{0}}^{*}$, for $g \in \mathbf{G}_{0}$.

The ideal sheaf $\mathscr{I}_{M}$ is generated, as a $C_{M}^{\infty}$ module, by the forms $\eta^{*}$, for $\eta$ varying in the annihilator $\mathfrak{q}^{0}=\left\{\eta \in \mathfrak{g}^{*} \mid \eta^{*}(Z)=0, \forall Z \in \mathfrak{q}\right\}$ of $\mathfrak{q}$ in $\mathfrak{g}^{*}$. 
Definition 3.4. A $C R$ algebra is a pair $\left(\mathfrak{g}_{0}, \mathfrak{q}\right)$, consisting of a real Lie algebra $\mathfrak{g}_{0}$ and of a complex Lie subalgebra $\mathfrak{q}$ of its complexification $\mathfrak{g}$, such that the quotient $\mathfrak{g}_{0} /\left(\mathfrak{q} \cap \mathfrak{g}_{0}\right)$ is a finite dimensional real vector space.

If $M$ is a $\mathbf{G}_{0}$-homogeneous $C R$ manifold and $\mathfrak{q}$ is defined by (3.1), we say that the $C R$ algebra $\left(\mathfrak{g}_{0}, \mathfrak{q}\right)$ is associated to $M$ at $p_{0}$.

Remark 3.5. If ( $\left.\mathfrak{g}_{0}, \mathfrak{q}\right)$ is associated to $M$ at $p_{0}$, and $g \in \mathbf{G}_{0}$, then $\left(\mathfrak{g}_{0}, \operatorname{Ad}_{\mathfrak{g}}(g)(\mathfrak{q})\right)$ is associated to $M$ at $g \cdot p_{0}$.

Remark 3.6. The $C R$-dimension and $C R$-codimension of $M$ can be computed in terms of its associated $C R$ algebra $\left(\mathfrak{g}_{0}, \mathfrak{q}\right)$. We have indeed

$$
C R-\operatorname{dim} M=\operatorname{dim}_{\mathbb{C}} \mathfrak{q}-\operatorname{dim}_{\mathbb{C}}(\mathfrak{q} \cap \overline{\mathfrak{q}}), \quad C R-\operatorname{codim} M=\operatorname{dim}_{\mathbb{C}} \mathfrak{g}-\operatorname{dim}_{\mathbb{C}}(\mathfrak{q}+\overline{\mathfrak{q}}) .
$$

Definition 3.7. Let $\mathbf{G}_{0}$ be a real form of a complex Lie group $\mathbf{G}$. We say that a $\mathbf{G}_{0}$-homogeneous $C R$ manifold $M$ is $\mathbf{G}$-realizable if $M$ admits a $\mathbf{G}_{0}$-equivariant generic $C R$-embedding into a complex homogeneous space $N$ of $\mathbf{G}$.

Remark 3.8. Let $M$ be a $\mathbf{G}_{0}$-homogeneous $C R$ manifold, with isotropy $\mathbf{M}_{0}$ and $C R$ algebra $\left(\mathfrak{g}_{0}, \mathfrak{q}\right)$ at $p_{0} \in M$. Then $M$ is $\mathbf{G}$-realizable if and only if there is a closed complex Lie subgroup $\mathbf{Q}$ of $\mathbf{G}$ with $\operatorname{Lie}(\mathbf{Q})=\mathfrak{q}$ and $\mathbf{Q} \cap \mathbf{G}_{0}=\mathbf{M}_{0}$.

Notation 3.9. For a linear subspace $\mathfrak{I}$ of a Lie algebra $\mathfrak{g}$, we will denote by $\mathscr{L}(\mathfrak{l})$ the Lie subalgebra of $\mathfrak{g}$ generated by $\mathrm{l}$ :

$$
\mathscr{L}(\mathfrak{l})=\mathfrak{l}+[\mathfrak{l}, \mathfrak{l}]+[\mathfrak{l},[\mathfrak{l}, \mathfrak{l}]]+\cdots .
$$

Let $M, N$ be $\mathbf{G}_{0}$-homogeneous $C R$ manifolds and $\phi: M \rightarrow N$ a $\mathbf{G}_{0}$-equivariant smooth map. Fix $p_{0} \in M$ and let $\left(\mathfrak{g}_{0}, \mathfrak{q}\right)$ be the $C R$ algebra of $M$ at $p_{0},\left(\mathfrak{g}_{0}, \mathfrak{e}\right)$ the $C R$ algebra of $N$ at $\phi\left(p_{0}\right)$, respectively. Then

$$
\mathfrak{q} \cap \bar{q} \subset \mathfrak{e} \cap \bar{e}
$$

and we have

Proposition 3.10. The $\mathbf{G}_{0}$-equivariant map $M \stackrel{\phi}{\longrightarrow} N$ is $C R$ if and only if $\mathfrak{q} \subset \mathrm{e}$. Assume $M \stackrel{\phi}{\longrightarrow} N$ is $C R$. Then:

(3.5) $\phi$ is a CR-submersion if and only if $\mathrm{e}=\mathfrak{q}+\mathrm{e} \cap \overline{\mathrm{e}}$.

(3.6) $\phi$ is a CR-spread if and only if $\mathrm{e}=\mathscr{L}(\mathfrak{q}+\mathrm{e} \cap \overline{\mathrm{e}})$.

(3.7) The fibers of $M \stackrel{\phi}{\longrightarrow} N$ are totally real if and only if $\mathfrak{q} \cap \overline{\mathrm{e}}=\mathrm{e} \cap \overline{\mathrm{q}}=\mathfrak{q} \cap \overline{\mathrm{q}}$.

The fibers of $M \stackrel{\phi}{\longrightarrow} N$ are totally complex if and only if

$$
\mathfrak{q} \cap \overline{\mathfrak{e}}+\mathfrak{e} \cap \overline{\mathfrak{q}}=\mathfrak{e} \cap \overline{\mathrm{e}} .
$$

Proof. For (3.5), (3.7), (3.8) we refer to [18].

Let us prove (3.6). Denote by $\mu, v$ the $C R$ structures of $M, N$, respectively. The map $\phi$ fits into a commutative diagram

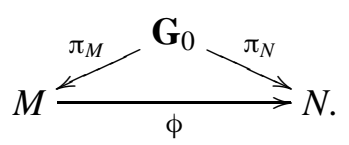

It is convenient here to identify the elements of $\mathfrak{g}$ to the corresponding left-invariant complex vector fields on $\mathbf{G}_{0}$. Then $\pi_{M}^{*} \mathscr{Z}_{\mu}$ and $\pi_{N}^{*} \mathscr{Z}_{v}$ are the distributions of complex vector fields on $\mathbf{G}_{0}$ which are generated by the elements of $\mathfrak{q}, \mathfrak{e}$, respectively, 
and, to verify that (3.6) is necessary and sufficient for $\phi$ being a $C R$ spread, it suffices to note that $\pi_{M}^{*}\left(\mathscr{L}\left(\mathscr{Z}_{\mu}+\mathscr{V}_{\phi}\right)\right)=C_{\mathbf{G}_{0}}^{\infty} \otimes \mathscr{L}(\mathfrak{q}+\mathfrak{e} \cap \overline{\mathrm{e}})$.

Proposition 3.11. Let $M$ be a $\mathbf{G}_{0}$-homogeneous smooth manifold, with isotropy $\mathbf{M}_{0}$ at $p_{0} \in M$, and $\mu_{1}, \mu_{2}$ two $\mathbf{G}_{0}$-equivariant $C R$ structures on $M$. Let $\left(\mathfrak{g}_{0}, \mathfrak{q}_{1}\right)$, $\left(\mathfrak{g}_{0}, \mathfrak{q}_{2}\right)$ be the CR algebras at $p_{0}$ corresponding to $\mu_{1}, \mu_{2}$, respectively. Then

$$
\mathfrak{q}_{1} \cap \overline{\mathfrak{q}}_{1}=\mathfrak{q}_{2} \cap \overline{\mathfrak{q}}_{2}=\mathfrak{m} \text {, with } \mathfrak{m}=\mathbb{C} \otimes \operatorname{Lie}\left(\mathbf{M}_{0}\right),
$$

and a necessary and sufficient condition for $\mu_{2}$ to strengthen $\mu_{1}$ is that

$$
\mathfrak{q}_{1} \subset \mathfrak{q}_{2} .
$$

The notion of strengthening a $C R$ structure translates into a corresponding notion for $C R$ algebras:

Definition 3.12. We say that $\left(\mathrm{g}_{0}, \mathfrak{q}_{2}\right)$ strengthens $\left(\mathfrak{g}_{0}, \mathfrak{q}_{1}\right)$ if $(3.9)$ and $(3.10)$ are satisfied.

\section{COMPaCt HOMOgENEOUS $C R$ MANIFOLDS AND h-REDUCTIVENESS}

4.1. Splittable subalgebras of a complex reductive Lie algebra. Let $\kappa$ be a reductive complex Lie algebra, and

$$
\kappa=\mathfrak{\jmath} \mathfrak{s}, \quad \mathfrak{z}=\{X \in \kappa \mid[X, \kappa]=\{0\}\}, \quad \mathfrak{s}=[\kappa, \kappa]
$$

its decomposition into the direct sum of its center and its semisimple ideal. We say that $X \in \kappa$ is semisimple if $\operatorname{ad}(X)$ is a semisimple derivation of $\kappa$, and nilpotent if $X \in \mathfrak{s}$ and $\operatorname{ad}(X)$ is nilpotent.

An equivalent formulation is obtained by considering a faithful matrix representation of $\kappa$ in which the elements of $z$ are diagonal. Semisimple and nilpotent elements correspond to semisimple and nilpotent matrices, respectively.

Each $X \in \kappa$ admits a unique Jordan-Chevalley decomposition

$$
X=X_{s}+X_{n}, \quad \text { with } X_{s} \text { semisimple, } X_{n} \text { nilpotent, and }\left[X_{s}, X_{n}\right]=0 .
$$

A Lie subalgebra $\mathfrak{v}$ of $\kappa$ is splittable if, for each $X \in \mathfrak{v}$, both $X_{s}$ and $X_{n}$ belong to $\mathfrak{v}$.

If $\mathfrak{v}$ is a Lie subalgebra of $\kappa$, the nilpotent elements of its radical $\operatorname{rad}(\mathfrak{v})$ form a nilpotent ideal $\mathfrak{n}_{\kappa}(\mathfrak{v})$ of $\mathfrak{v}$, with

$$
\operatorname{rad}_{\mathfrak{n}}(\mathfrak{v})=\operatorname{rad}(\mathfrak{v}) \cap[\mathfrak{v}, \mathfrak{p}] \subset \mathfrak{n}_{\kappa}(\mathfrak{v}) \subset \operatorname{nil}(\mathfrak{v}),
$$

where nil(v) is the nilradical, i.e. the maximal nilpotent ideal of $\mathfrak{v}$, and $\operatorname{rad}_{\mathfrak{n}}(\mathfrak{v})$ its nilpotent radical, i.e. the intersection of the kernels of all irreducible finite dimensional linear representations of $\mathfrak{v}$. Note that the nilpotent ideal $\mathfrak{n}_{\kappa}(\mathfrak{v})$, unlike $\operatorname{nil}(\mathfrak{v})$ and $\operatorname{rad}_{\mathfrak{n}}(\mathfrak{v})$, depends on the inclusion $\mathfrak{v} \subset \kappa(\mathrm{cf}$. [8, §5.3]).

We recall (see $[8, \S 5.4])$

Proposition 4.1. Every splittable Lie subalgebra $\mathfrak{v}$ admits a Levi-Chevalley decomposition

$$
\mathfrak{v}=\mathfrak{n}_{\kappa}(\mathfrak{v}) \oplus \mathfrak{m},
$$

where $\mathrm{m}$ is reductive in $\kappa$, and is uniquely determined modulo conjugation by elementary automorphisms of $\mathfrak{v}$, i.e. finite products of automorphisms of the form $\exp (\operatorname{ad}(X))$, with $X \in \mathfrak{v}$ and nilpotent. 
4.2. Definition of $n$-reductive. Let $\kappa$ be the complexification of a compact Lie algebra $\kappa_{0}$. Conjugation in $\kappa$ is understood with respect to the real form $\kappa_{0}$. Note that all subalgebras of a compact Lie algebra are compact and hence reductive.

Proposition 4.2. For any complex Lie subalgebra $\mathfrak{v}$ of $\kappa$, the intersection $\mathfrak{v} \cap \overline{\mathfrak{v}}$ is reductive and splittable. In particular, $\mathfrak{v} \cap \overline{\mathfrak{v}} \cap \mathfrak{n}_{\kappa}(\mathfrak{v})=\{0\}$. A splittable $\mathfrak{v}$ admits $a$ Levi-Chevalley decomposition with a reductive Levi factor containing $\mathfrak{v} \cap \overline{\mathfrak{v}}$.

Proof. We recall that $\mathfrak{v}$ is splittable if and only if $\operatorname{rad}(\mathfrak{v})$ is splittable. When this is the case, $\mathfrak{v}$ admits a Levi-Chevalley decomposition, and all maximal reductive Lie subalgebras of $\mathfrak{v}$ can be taken as reductive Levi factors. The intersection $\mathfrak{v} \cap \overline{\mathfrak{v}}$ is reductive, because it is the complexification of the compact Lie algebra $\mathfrak{v} \cap \kappa_{0}$. Then, the reductive Levi factor in the Levi-Chevalley decomposition of $\mathfrak{v}$ can be taken to contain $\mathfrak{v} \cap \overline{\mathfrak{v}}$ (see e.g. [22]).

Let $\mathbf{K}_{0}$ be a compact Lie group with Lie algebra $\kappa_{0}$ and $M$ a $\mathbf{K}_{0}$-homogeneous $C R$ manifold, with isotropy $\mathbf{M}_{0}$ and $C R$ algebra $\left(\kappa_{0}, \mathfrak{v}\right)$ at a point $p_{0} \in M$.

Definition 4.3. We say that $M$, and the $C R$ algebra $\left(\kappa_{0}, \mathfrak{v}\right)$, are $\mathfrak{n}$-reductive if $\mathfrak{v} \cap \overline{\mathfrak{v}}$ is a reductive complement of $\mathfrak{n}_{\kappa}(\mathfrak{v})$ in $\mathfrak{v}$.

Remark 4.4. If $\left(\kappa_{0}, \mathfrak{v}\right)$ is $\mathfrak{n}$-reductive, then $\mathfrak{v}$ is splittable. Indeed all elements of $\mathfrak{n}_{\kappa}(\mathfrak{v})$ are nilpotent and all elements of $\mathfrak{v} \cap \overline{\mathfrak{v}}$ are splittable, because this subalgebra is the complexification of the subalgebra $\mathfrak{v} \cap \kappa_{0}$, which is splittable because consists of semisimple elements.

Theorem 4.5 (realization). Every $\mathfrak{n}$-reductive $\mathbf{K}_{0}$-homogeneous $C R$ manifold is K-realizable (see Definition 3.7).

In fact, if $M$ is a $\mathbf{K}_{0}$-homogeneous $C R$ manifold with isotropy $\mathbf{M}_{0}$ and $C R$ algebra $\left(\kappa_{0}, \mathfrak{v}\right)$ at $p_{0} \in M$, then there is a closed complex subgroup $\mathbf{V}$ of $\mathbf{K}$ with $\operatorname{Lie}(\mathbf{V})=\mathfrak{v}$ and $\mathbf{V} \cap \mathbf{K}_{0}=\mathbf{M}_{0}$. Set $\mathrm{m}_{0}=\operatorname{Lie}\left(\mathbf{M}_{0}\right), \mathfrak{m}=\mathbb{C} \otimes_{\mathbb{R}} \mathrm{m}_{0}$.

The Lie subgroup $\mathbf{V}$ admits a Levi-Chevalley decomposition

$$
\mathbf{V}=\mathbf{V}_{n} \mathbf{M}, \quad \text { with } \operatorname{Lie}\left(\mathbf{V}_{n}\right)=\mathfrak{n}_{\kappa}(\mathfrak{v}), \quad \operatorname{Lie}(\mathbf{M})=\mathfrak{m} .
$$

Proof. The linear subspace $i \mathrm{~m}_{0}$ of $\kappa$ is $\operatorname{Ad}_{\kappa}\left(\mathbf{M}_{0}\right)$-invariant, and the unique complexification $\mathbf{M}$ of $\mathbf{M}_{0}$ in $\mathbf{K}$ is defined by

$$
\mathbf{M}=\left\{g \exp (i X) \mid g \in \mathbf{M}_{0}, X \in \mathfrak{m}_{0}\right\} .
$$

Clearly Lie $(\mathbf{M})=m$. The complex analytic Lie subgroup $\mathbf{V}_{n}$ of $\mathbf{K}$ with Lie algebra $\mathfrak{n}_{\kappa}(\mathfrak{v})$ is closed, is normalized by $\mathbf{M}$ and $\mathbf{V}_{n} \cap \mathbf{M}$ is finite. Hence

$$
\mathbf{V}=\mathbf{V}_{n} \mathbf{M}=\left\{g_{1} g_{2} \mid g_{1} \in \mathbf{V}_{n}, g_{2} \in \mathbf{M}\right\}
$$

is a closed Lie subgroup of $\mathbf{K}$, with $\mathbf{V} \cap \mathbf{K}_{0}=\mathbf{M}_{0}$ and $\operatorname{Lie}(\mathbf{V})=\mathfrak{v}$. With $V=\mathbf{K} / \mathbf{V}$, the inclusion $\mathbf{K}_{0} \hookrightarrow \mathbf{K}$ passes to the quotients, defining a $\mathbf{K}_{0}$-equivariant $C R$ embedding $M \hookrightarrow V$.

Later, in \$5 we will encounter a large class of n-reductive compact homogeneous $C R$ manifolds. We exhibit here an example of a compact homogeneous $C R$ manifold $M$ which is not n-reductive.

Example 4.6. Let $\mathbf{K}_{0}=\mathbf{S} \mathbf{U}(n), n \geq 3$. Fix a complex symmetric non degenerate $n \times n$ matrix $S$ and consider the subgroup $\mathbf{V}=\left\{a \in \mathbf{S L}(n, \mathbb{C}) \mid a^{t} S a=S\right\}$ of $\mathbf{S L}(n, \mathbb{C})$, with Lie algebra $\mathfrak{v}=\left\{X \in \mathfrak{s l}(n, \mathbb{C}) \mid X^{t} S+S X=0\right\}$. Set $\mathbf{M}_{0}=\mathbf{V} \cap \mathbf{K}_{0}$ 
and $M=\mathbf{K}_{0} / \mathbf{M}_{0}$. This is a $\mathbf{K}_{0}$-homogeneous $C R$ manifold with $C R$ algebra $\left(\kappa_{0}, \mathfrak{v}\right)$, where $\kappa_{0} \simeq \mathfrak{s u}(n), \mathfrak{v} \simeq \mathfrak{s} \mathfrak{p}(n, \mathbb{C})$. If $S, S^{*}$ are linearly independent, then $\mathfrak{v}$ is a semisimple Lie subalgebra of $\kappa$ distinct from $\mathfrak{v} \cap \overline{\mathfrak{v}}$.

4.3. Regular and parabolic subalgebras. Let $t_{0}$ be a maximal torus of $\kappa_{0}$.

Definition 4.7. We call $t_{0}$-regular a subalgebra $\mathfrak{v}$ of $\kappa$ which is normalized by $t_{0}$.

The complexification $t$ of $t_{0}$ is a Cartan subalgebra of $\kappa$. Denote by $\mathcal{K}$ the root system of $(\kappa, t)$ and by

$$
\kappa^{\alpha}=\{X \in \kappa \mid[H, X]=\alpha(H) X, \forall H \in \mathrm{t}\}
$$

the root space of $\alpha \in \mathcal{K}$. Note that $\overline{\kappa^{\alpha}}=\kappa^{-\alpha}$.

Lemma 4.8. $A \mathrm{t}_{0}$-regular subalgebra $\mathfrak{v}$ of $\mathrm{\kappa}$ is splittable and admits a Levi-Chevalley decomposition

$$
\mathfrak{v}=\mathfrak{n}_{\kappa}(\mathfrak{v}) \oplus \mathfrak{m} \text {, with reductive Levi factor } \mathfrak{m}=\mathrm{t} \cap \mathfrak{v}+\mathfrak{v} \cap \overline{\mathfrak{v}} .
$$

In particular, $\left(\kappa_{0}, \mathfrak{v}\right)$ is $\mathfrak{n}$-reductive if and only if $\mathrm{t} \cap \mathfrak{v} \subset \overline{\mathfrak{v}}$.

Proof. A t $t_{0}$-regular $\mathfrak{v}$ admits a root-space decomposition

$$
\mathfrak{v}=\mathfrak{a} \oplus \sum_{\alpha \in \mathcal{V}} \kappa^{\alpha} \text {, with } \mathfrak{a}=\mathrm{Z}_{\mathfrak{v}}(\mathrm{t})=\mathrm{t} \cap \mathfrak{v}, \mathcal{V}=\left\{\alpha \in \mathcal{K} \mid \kappa^{\alpha} \subset \mathfrak{v}\right\} .
$$

Thus the Lie algebra $\mathfrak{v}$ admits a set of generators that are either semisimple or nilpotent and is splittable by [8, Ch.VII, §5].

The conjugation with respect to the real form $\kappa_{0}$ yields by duality the conjugation $\bar{\alpha}=-\alpha$ in $\mathcal{K}$. Set

$$
\begin{aligned}
\mathfrak{n}_{\kappa}(\mathfrak{v})=\sum_{\alpha \in \mathcal{V}_{n}} \kappa^{\alpha}, & \text { with } \mathcal{V}_{n}=\{\alpha \in \mathcal{V} \mid-\alpha \notin \mathcal{V}\}, \\
\mathfrak{m}=\mathfrak{a}+\mathfrak{v} \cap \overline{\mathfrak{v}}=\mathfrak{a} \oplus \sum_{\alpha \in \mathcal{V}_{r}} \kappa^{\alpha}, & \text { with } \mathcal{V}_{r}=\{\alpha \in \mathcal{V} \mid-\alpha \in \mathcal{V}\}=\mathcal{V} \cap \overline{\mathcal{V}},
\end{aligned}
$$

and (4.4) follows, as one easily checks that $\mathfrak{n}_{\kappa}(\mathfrak{v})$ is the ideal of nilpotent elements of $\operatorname{rad}(\mathfrak{v})=\mathfrak{a} \oplus \mathfrak{n}_{\kappa}(\mathfrak{v})$ and $\mathfrak{m}$ is reductive.

If $A \in \kappa_{0}$, then $\operatorname{ad}_{\kappa}(A)$ is semisimple with purely imaginary eigenvalues. The sum of the corresponding eigenspaces with positive imaginary part

$$
\mathfrak{q}_{A}=\sum_{\lambda \geq 0}\{X \in \kappa \mid[A, X]=i \lambda X\}
$$

is a parabolic Lie subalgebra of $\kappa$, and all parabolic subalgebras of $\kappa$ have this form. The intersection of $q_{A}$ with $\kappa_{0}$ is the centralizer of $A$ in $\kappa_{0}$ :

$$
\mathfrak{S}_{0}\left(\mathfrak{q}_{A}\right)=\mathfrak{q}_{A} \cap \kappa_{0}=\mathrm{Z}_{\kappa_{0}}(A)=\left\{X \in \kappa_{0} \mid[A, X]=0\right\}
$$

and is the union of the maximal tori of $\kappa_{0}$ contained in $\mathfrak{q}_{A}$. Its complexification is the centralizer of $A$ in $\kappa$ and equals the intersection with its $\kappa_{0}$-conjugate:

$$
\mathfrak{L}\left(\mathfrak{q}_{A}\right)=Z_{\kappa}(A)=\mathfrak{q}_{A} \cap \overline{\mathfrak{q}}_{A} .
$$

This subalgebra $\mathfrak{L}\left(\mathfrak{q}_{A}\right)$ is the unique conjugation invariant reductive Levi factor in the Levi-Chevalley decomposition

$$
\mathfrak{q}_{A}=\mathfrak{n}_{\kappa}\left(\mathfrak{q}_{A}\right) \oplus \mathfrak{L}\left(\mathfrak{q}_{A}\right)=\mathfrak{n}_{\kappa}\left(\mathfrak{q}_{A}\right) \oplus \mathfrak{q}_{A} \cap \overline{\mathfrak{q}}_{A} .
$$

Notation 4.9. In the following, for $\mathfrak{v}$ complex subalgebra of $\kappa$, we shall use the notation

$$
\mathfrak{L}_{0}(\mathfrak{v})=\mathfrak{v} \cap \kappa_{0}, \quad \mathfrak{L}(\mathfrak{v})=\mathfrak{v} \cap \overline{\mathfrak{v}} .
$$

We denote by $\mathbb{P}_{\kappa}$ the set of complex parabolic Lie subalgebras of $\kappa$. 
Remark 4.10. If $\mathfrak{q} \in \mathbb{P}_{\kappa}$, then the set $\left\{A \in \kappa_{0} \mid \mathfrak{q}_{A}=\mathfrak{q}\right\}$ is an open cone in the real vector space $\mathrm{Z}_{\kappa_{0}}\left(\mathfrak{Q}_{0}(\mathfrak{q})\right)=\left\{A \in \kappa_{0} \mid[A, \mathfrak{q} \cap \bar{q}]=\{0\}\right\}$.

Lemma 4.11. If $\mathfrak{q} \in \mathbb{P}_{\kappa}$, then $\mathfrak{n}_{\kappa}(\mathfrak{q})=\operatorname{rad}_{\mathfrak{n}}(\mathfrak{q})$ and $\left(\kappa_{0}, \mathfrak{q}\right)$ is $\mathfrak{n}$-reductive and totally complex.

Proof. An element $\mathfrak{q} \in \mathbb{P}_{\kappa}$ contains a a maximal torus $t_{0}$ of $\kappa_{0}$ (see e.g. [8, Ch.VIII]). Let $\mathrm{t}=\mathbb{C} \otimes_{\mathbb{R}} \mathrm{t}_{0}$ be its complexification, $\mathcal{K}$ the root system of $(\kappa, \mathrm{t})$ and $Q=\left\{\alpha \in \mathcal{K} \mid \kappa^{\alpha} \subset \mathfrak{q}\right\}$ the parabolic set of $\mathfrak{q}$. With $Q_{r}=\{\alpha \in Q \mid-\alpha \in Q\}=Q \cap \bar{Q}$, $Q_{n}=\{\alpha \mid-\alpha \notin Q\}=Q \backslash \bar{Q}$, we have

$$
\mathfrak{q}=\mathfrak{n}_{\kappa}(\mathfrak{q}) \oplus \mathfrak{L}(\mathfrak{q}), \text { with } \mathfrak{n}_{\kappa}(\mathfrak{q})=\sum_{\alpha \in Q_{n}} \kappa^{\alpha}, \quad \mathfrak{L}(\mathfrak{q})=\mathfrak{t} \oplus \sum_{\alpha \in Q_{r}} \kappa^{\alpha} .
$$

Since

$$
\overline{n_{\kappa}(\mathfrak{q})}=\sum_{\alpha \in Q_{n}} \overline{\kappa^{\alpha}}=\sum_{\alpha \in Q_{n}} \kappa^{-\alpha},
$$

and $Q \cup(-Q)=\mathcal{K}$ because $Q$ is parabolic, we obtain

$$
\kappa=\mathfrak{n}_{\kappa}(\mathfrak{q}) \oplus \mathfrak{L}(\mathfrak{q}) \oplus \overline{\mathfrak{n}_{\kappa}(\mathfrak{q})}=\mathfrak{q}+\overline{\mathfrak{q}},
$$

showing that $\left(\kappa_{0}, q\right)$ is totally complex (see e.g. [18, p.237]).

4.4. Morphisms of n-reductive $C R$ algebras. Let $M$ be a $\mathbf{K}_{0}$-homogeneous $C R$ manifold with isotropy subgroup $\mathbf{M}_{0}$ and $C R$ algebra $\left(\kappa_{0}, \mathfrak{v}\right)$ at $p_{0} \in M$. If $M$ is $\mathfrak{n}$ reductive, we can identify $T_{p_{0}}^{0,1} M$ with $\mathfrak{n}_{\kappa}(\mathfrak{v})$. Therefore, a $\kappa_{0}$-equivariant morphism of $\mathfrak{n}$-reductive $C R$ algebras $\left(\kappa_{0}, \mathfrak{p}\right) \rightarrow\left(\kappa_{0}, \mathfrak{q}\right)$ induces a $\mathbb{C}$-linear map $\mathfrak{n}_{\kappa}(\mathfrak{p}) \rightarrow \mathfrak{n}_{\kappa}(\mathfrak{q})$, which is defined by the commutative diagram

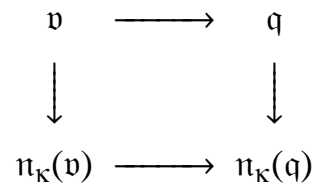

in which the top horizontal arrow is inclusion and the two vertical arrows are projections along $\mathfrak{v} \cap \overline{\mathfrak{v}}$ and $\mathfrak{q} \cap \overline{\mathfrak{q}}$, respectively.

Proposition 4.12. Let $\mathfrak{v}, \mathfrak{q}$ be complex Lie subalgebras of $\kappa$, with $\mathfrak{v} \subset \mathfrak{q}$, and assume that $\left(\kappa_{0}, \mathfrak{v}\right)$ is $\mathfrak{n}$-reductive. Set $\mathfrak{L}(\mathfrak{v})=\mathfrak{v} \cap \overline{\mathfrak{v}}, \mathfrak{L}(\mathfrak{q})=\mathfrak{q} \cap \overline{\mathfrak{q}}$. The $\kappa_{0}$-equivariant fibration

$$
\left(\kappa_{0}, \mathfrak{v}\right) \longrightarrow\left(\kappa_{0}, \mathfrak{q}\right)
$$

- is a CR-submersion if and only if $\mathfrak{q}=\mathfrak{n}_{\mathrm{K}}(\mathfrak{v})+\mathfrak{L}(\mathfrak{q})$;

- is a CR-submersion with totally real fibers if and only if $\mathfrak{q}=\mathfrak{n}_{\kappa}(\mathfrak{p}) \oplus \mathfrak{L}(\mathfrak{q})$.

In particular,

- is a CR-submersion if $\mathfrak{n}_{\mathrm{K}}(\mathfrak{q}) \subset \mathfrak{n}_{\mathrm{K}}(\mathfrak{v})$;

- is a CR-submersion with totally real fibers if $\mathfrak{n}_{\mathrm{K}}(\mathfrak{q})=\mathfrak{n}_{\mathrm{K}}(\mathfrak{v})$.

Proof. If $\left(\kappa_{0}, \mathfrak{v}\right)$ is $\mathfrak{n}$-reductive, we have

$$
\mathfrak{v}+\mathfrak{L}(\mathfrak{q})=\mathfrak{n}_{\kappa}(\mathfrak{p})+\mathfrak{L}(\mathfrak{q})
$$

because $\mathfrak{L}(\mathfrak{p}) \subset \mathfrak{L}(\mathfrak{q})$. In view of $(3.5)$, this equality yields the above characterization of $C R$-submersion from a n-reductive $C R$ algebra.

If $\mathfrak{q}=\mathfrak{n}_{\kappa}(\mathfrak{v}) \oplus \mathfrak{Q}(\mathfrak{q})$, then $\overline{\mathfrak{q}}=\overline{\mathfrak{n}_{\kappa}(\mathfrak{v})} \oplus \mathfrak{Q}(\mathfrak{q})$ and we have a direct sum decomposition

$$
\mathfrak{q}+\overline{\mathfrak{q}}=\mathfrak{n}_{\kappa}(\mathfrak{p}) \oplus \mathfrak{L}(\mathfrak{q}) \oplus \overline{\mathfrak{n}_{K}(\mathfrak{v})} .
$$


An element $X \in \mathfrak{v} \cap \bar{q}$ uniquely decomposes as sums $X=X_{1}+X_{2}=Y_{1}+Y_{2}$ with $X_{1} \in \mathfrak{n}_{\kappa}(\mathfrak{v}), X_{2} \in \mathfrak{L}(\mathfrak{v}) \subset \mathfrak{L}(\mathfrak{q}), Y_{1} \in \mathfrak{L}(\mathfrak{q}), Y_{2} \in \overline{\mathfrak{n}_{\kappa}(\mathfrak{v})}$. By comparing the two decompositions, we obtain that $X_{1}=Y_{2}=0$ and $X=Y_{1}=X_{2} \in \mathfrak{L}(\mathfrak{v})$.

Vice versa, if $\mathfrak{v} \cap \overline{\mathfrak{q}}=\mathfrak{L}(\mathfrak{v})$, then $\mathfrak{n}_{\kappa}(\mathfrak{v}) \cap \mathfrak{L}(\mathfrak{q})=\{0\}$ and the sum $\mathfrak{n}_{\kappa}(\mathfrak{v})+\mathfrak{L}(\mathfrak{q})$ is direct.

Example 4.13. Let us consider the $C R$ algebras $\left(\mathfrak{u}_{3}, \mathfrak{v}\right)$ and $\left(\mathfrak{u}_{3}, \mathfrak{q}\right)$ with

$$
\begin{aligned}
& \mathfrak{v}=\left\{\left(\begin{array}{ccc}
\lambda & \zeta_{1} & \zeta_{2} \\
0 & \lambda & \zeta_{1} \\
0 & 0 & \lambda
\end{array}\right) \mid \lambda, \zeta_{1}, \zeta_{2} \in \mathbb{C}\right\}, \\
& \mathfrak{q}=\left\{\left(\begin{array}{ccc}
\lambda_{1} & \zeta_{1} & \zeta_{2} \\
0 & \lambda_{2} & \zeta_{3} \\
0 & \zeta_{4} & \lambda_{3}
\end{array}\right) \mid \lambda_{1}, \lambda_{2}, \lambda_{3}, \zeta_{1}, \zeta_{2}, \zeta_{4}, \zeta_{2} \in \mathbb{C}\right\} .
\end{aligned}
$$

We have

$$
\mathfrak{n}_{\kappa}(\mathfrak{v})=\left\{\left(\begin{array}{ccc}
0 & \zeta_{1} & \zeta_{2} \\
0 & 0 & \zeta_{1} \\
0 & 0 & 0
\end{array}\right)\right\}, \quad \mathfrak{n}_{\kappa}(\mathfrak{q})=\left\{\left(\begin{array}{ccc}
0 & \zeta_{1} & \zeta_{2} \\
0 & 0 & 0 \\
0 & 0 & 0
\end{array}\right)\right\},
$$

so that $\mathfrak{q}=\mathfrak{n}_{\kappa}(\mathfrak{v}) \oplus \mathfrak{L}(\mathfrak{q})$, showing that $\left(\mathfrak{u}_{3}, \mathfrak{p}\right) \rightarrow\left(\mathfrak{u}_{3}, \mathfrak{q}\right)$ is a $C R$-submersion with totally real fibers, for which $\mathfrak{n}_{\kappa}(\mathfrak{q}) \not \subset n_{\kappa}(\mathfrak{v})$.

\subsection{Regularity type for $n$-reductive $C R$ algebras.}

Definition 4.14. We say that an $\mathfrak{n}$-reductive $C R$ algebra $\left(\kappa_{0}, \mathfrak{v}\right)$ is of

- type $\mathrm{I}$ if $\mathfrak{v}$ is $\mathrm{t}_{0}$-regular, for some maximal torus $t_{0}$ of $\kappa_{0}$,

- type II if $\mathfrak{L}(\mathfrak{v})$ is $t_{0}$-regular, for some maximal torus $t_{0}$ of $\kappa_{0}$,

- type III, or general, if we make no assumption on normalizing tori.

4.6. Minimal orbits of compact forms. Let $\mathbf{K}$ be a connected reductive complex Lie group, with $\operatorname{Lie}(\mathbf{K})=\kappa$. If $\mathbf{V}$ is an algebraic subgroup of $\mathbf{K}$, then $\mathbf{V}$ admits a Levi-Chevalley decomposition

$$
\mathbf{V}=\mathbf{V}_{n} \mathbf{V}_{r} \text {, with } \mathbf{V}_{n} \text { unipotent, } \mathbf{V}_{r} \text { reductive, }\left|\mathbf{V}_{n} \cap \mathbf{V}_{r}\right|<\infty \text {. }
$$

The maximal compact subgroup $\mathbf{M}_{0}$ of $\mathbf{V}_{r}$ is contained in a compact form $\mathbf{K}_{0}$ of $\mathbf{K}$. The orbit $M$ of $\mathbf{K}_{0}$ through the base point of the complex homogeneous space $V=\mathbf{K} / \mathbf{V}$ inherits from $V$ the structure of a $\mathbf{K}_{0}$-homogeneous n-reductive $C R$ manifold, with isotropy $\mathbf{M}_{0}$ and $C R$ algebra $\left(\kappa_{0}, \mathfrak{v}\right)$ at the base point, where $\kappa_{0}=\operatorname{Lie}\left(\mathbf{K}_{0}\right)$ and $\mathfrak{v}=\operatorname{Lie}(\mathbf{V})$. Since all compact forms of $\mathbf{K}$ are conjugated by an inner automorphism, we obtain the

Proposition 4.15. Let $V$ be the homogeneous complex manifold obtained as the quotient of a connected reductive complex manifold $\mathbf{K}$ by an algebraic subgroup $\mathbf{V}$, and $\mathbf{K}_{0}$ a compact form of $\mathbf{K}$. Then $\mathbf{K}_{0} \backslash V$ contains $\mathfrak{n}$-reductive orbits. All $\mathrm{n}$-reductive orbits in $\mathbf{K}_{0} \backslash V$ are CR-diffeomorphic.

The $\mathfrak{n}$-reductive orbits are the orbits of minimal dimension in $\mathbf{K}_{0} \backslash V$.

Proof. We already proved existence. Let us prove equivalence. We can assume that the orbit $M$ through the base point $p_{0}$ is n-reductive. Let $p_{1}=g_{1} V, g_{1} \in \mathbf{K}$, and assume that also $M^{\prime}=K_{0} p_{1}$ is $\mathfrak{n}$-reductive. This means that $\mathbf{K}_{0} \cap \operatorname{ad}\left(g_{1}\right)(\mathbf{V})$ is a maximal compact subgroup of $\operatorname{ad}\left(g_{1}\right)(\mathbf{V})$, i.e. that $\operatorname{ad}\left(g_{1}^{-1}\right)\left(\mathbf{K}_{0}\right) \cap \mathbf{V}$ is a maximal compact subgroup of $\mathbf{V}$. By the conjugacy of maximal compact subgroups, there 
is an element $g_{2} \in \mathbf{V}$ such that $\operatorname{ad}\left(g_{1}^{-1}\right)\left(\mathbf{K}_{0}\right) \cap \mathbf{V}=\operatorname{ad}\left(g_{2}\right)\left(\mathbf{K}_{0}\right) \cap \mathbf{V}$. Then we obtain, for $k \in \mathbf{K}_{0}$,

$$
g_{1} \cdot g_{2} \cdot k \cdot p_{0}=\operatorname{ad}\left(g_{1} \cdot g_{2}\right)(k) \cdot g_{1} \cdot g_{2} \cdot p_{0}=k^{\prime} \cdot p_{1}, \quad \text { with } k^{\prime}=\operatorname{ad}\left(g_{1} \cdot g_{2}\right)(k) \in \mathbf{K}_{0} \text {. }
$$

This shows that the translation in $V$ by $g_{1} \cdot g_{2}$ transforms $M$ onto $M^{\prime}$. This map is a biholomorphism of $V$ and then its restriction to $M$ is a $C R$-diffeomorphism of $M$ onto $M^{\prime}$.

The fact that the n-reductive orbits have minimal dimension follows because the isotropy $\mathbf{M}_{p}$ of a $\mathbf{K}_{0}$-orbit $M$ at a point $p=g \cdot p_{0}$ is a reductive subgroup of $\operatorname{ad}(g)(\mathbf{V})$, and therefore $\operatorname{dim}_{\mathbb{R}} \mathbf{M}_{0} \leq \operatorname{dim}_{\mathbb{C}} \mathbf{V}_{r}$, and we have equality if and only if $\mathbf{M}_{0}$ is a maximal compact subgroup of $\operatorname{ad}(g)(\mathbf{V})$.

4.7. n-reductive structures on compact homogeneous spaces. Let $\mathbf{K}_{0}$ be a connected compact Lie group and $M$ a $\mathbf{K}_{0}$-homogeneous space, with isotropy $\mathbf{M}_{0}$ at $p_{0} \in M$. With $\operatorname{Lie}\left(\mathbf{M}_{0}\right)=\mathfrak{m}_{0}, \mathfrak{m}=\mathbb{C} \otimes_{\mathbb{R}} \mathfrak{m}_{0}$, the $C R$ algebra $\left(\kappa_{0}, \mathfrak{m}\right)$ defines on $M$ the trivial totally real $C R$ structure. In accordance with Theorem 4.5 , there is a reductive complex Lie subgroup $\mathbf{M}$ of the complexification $\mathbf{K}$ of $\mathbf{K}_{0}$ such that, with $M^{\mathbb{C}}=\mathbf{K} / \mathbf{M}$, we have a $\mathbf{K}_{0}$-equivariant immersion $M \hookrightarrow M^{\mathbb{C}}$ of $M$ into its complexification $M^{\mathbb{C}}$, which is Stein because $\mathbf{K}$ is Stein and $\mathbf{M}$ is the complexification of the compact subgroup $\mathbf{M}_{0}$ (see [16]).

We have

Proposition 4.16. The $\mathfrak{n}$-reductive $\mathbf{K}_{0}$-equivariant $C R$ structures on $M$ are in a one-to-one correspondence with the $\operatorname{Ad}_{\kappa}\left(\mathbf{M}_{0}\right)$-invariant complex subalgebras $\mathfrak{n}$ of $\kappa$ consisting of nilpotent elements.

If $\left(\kappa_{0}, \mathfrak{v}\right)$ is the $C R$ algebra at $p_{0}$ of an $\mathfrak{n}$-reductive $\mathbf{K}_{0}$-equivariant $C R$ structure on $M$, then, with the notation of Theorem 4.5 we have a commutative diagram

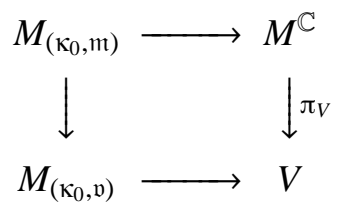

where $M_{\left(\mathrm{\kappa}_{0}, \mathrm{~m}\right)}$ and $M_{\left(\mathrm{\kappa}_{0}, \mathfrak{v}\right)}$ are the CR manifolds with underlying smooth manifold $M$ and $\mathbf{K}_{0}$-equivariant $C R$ structures defined by the CR algebras $\left(\kappa_{0}, \mathfrak{m}\right),\left(\kappa_{0}, \mathfrak{v}\right)$, respectively; the horizontal arrows are $\mathbf{K}$-realizations, the left vertical arrow is a strengthening of the CR structure, and the right vertical arrow is a holomorphic submersion with Euclidean fibers.

Proof. Indeed, the fibers of the $\mathbf{K}$-equivariant holomorphic submersion $M^{\mathbb{C}} \rightarrow V$ are complex nilmanifolds conjugate to $\mathbf{V}_{n}$.

Definition 4.17. We call the fibration $M^{\mathbb{C}} \stackrel{\pi_{V}}{\longrightarrow} V$ a Stein lift of $V$.

4.8. Complexification. Foliated complex manifolds. We keep the notation of $\$ 4.7$

Remark 4.18. The Stein lift of Definition 4.17 is characterized by the universal property that every $\mathbf{K}$-equivariant fibration $E \stackrel{\varpi}{\longrightarrow} V$ of a K-homogeneous Stein manifold $E$ onto $V$ factors through $M^{\mathbb{C}} \stackrel{\pi_{V}}{\longrightarrow} V$.

The fibers of $M^{\mathbb{C}} \stackrel{\pi_{V}}{\longrightarrow} V$ are the integral manifolds of a holomorphic distribution $\mathscr{D}_{\mathfrak{v}}$ on $M^{\mathbb{C}}$. 
Definition 4.19. We call the foliated complex manifold $\left(M^{\mathbb{C}}, \mathscr{D}_{\mathfrak{v}}\right)$ a complexification of the $C R$ manifold $M$.

This construction can be generalized to the case of a real analytic $C R$ manifold $M$. Indeed, $M$ has a complexification $M \stackrel{\iota_{M}}{\longrightarrow} M^{\mathbb{C}}$ and a generic embedding $M \stackrel{{ }^{V}}{\longrightarrow} V$ (see [6]). Since $l_{V}$ is real analytic, it extends uniquely to a holomorphic submersion $M^{\mathbb{C}} \stackrel{\pi_{V}}{\longrightarrow} V$ (after shrinking $V$ and $M^{\mathbb{C}}$, if needed). Since $M$ has in $M^{\mathbb{C}}$ a fundamental system of Stein neighborhoods, we can say that $M$ admits a complexification $\left(M^{\mathbb{C}}, \mathscr{D}\right)$ with a Stein $M^{\mathbb{C}}$ and $\mathscr{D}$ yielding a fibration on a generic complex neighborhood $V$ of $M$.

In this more general context, the leaves of $\mathscr{D}$ are also called Segre varieties of $M$.

For further reference, we collect below some general notions on foliated complex manifolds.

Definition 4.20. A foliated complex manifold is a pair $(E, \mathscr{D})$ consisting of a complex manifold $E$ and an integrable holomorphic distribution (of constant rank) $\mathscr{D}$ on $E$. We set $D_{p} E=\left\{X_{p} \mid X \in \mathscr{D}\right\} \subset T^{\mathbb{C}} E$, and $D E=\bigsqcup_{p \in E} D_{p} E$.

If $\left(E^{\prime}, \mathscr{D}^{\prime}\right)$ is another foliated complex manifold, a holomorphic map $\phi: E \rightarrow E^{\prime}$ is a $\mathscr{D}$-morphism if $\phi_{*}(D E) \subset D^{\prime} E^{\prime}$. We say that $\phi$ is

- a $\mathscr{D}$-submersion if $\phi_{*}\left(D_{p} E\right)=D_{\phi(p)}^{\prime} E^{\prime}$ for all $p \in E$;

- a $\mathscr{D}$-spread if it is a $\mathscr{D}$-submersion and $\phi^{*} \mathscr{D}^{\prime}$ is generated by $\mathscr{D}+\mathcal{V}_{\phi}$, where $\mathcal{V}_{\phi}$ is the vertical holomorphic distribution of $\phi$;

- a $\mathscr{D}$-deployment if it is a $\mathscr{D}$-spread and the maps $\phi_{*}(p): D_{p} E \rightarrow D_{\phi(p)}^{\prime} E^{\prime}$ are injective for all $p \in E$.

\section{A CLASS OF $\mathfrak{n}$-REDUCTIVE COMPACT HOMOGENEOUS $C R$ MANIFOLDS}

In this section we describe a large class of n-reductive compact homogeneous $C R$ manifold, which are $C R$ submanifolds of complex flag manifolds. Although it could be proved that these manifolds are n-reductive by using the results in $\$ 4.6$, we give a direct and more explicit proof of this fact.

5.1. Complex flag manifolds. Let $\mathbf{G}$ be a connected complex algebraic Lie group and $\mathbf{F}$ a parabolic subgroup of $\mathbf{G}$. If $\mathfrak{g}=\operatorname{Lie}(\mathbf{G}), \mathfrak{f}=\operatorname{Lie}(\mathbf{F})$, we have

$$
\mathbf{F}=\mathrm{N}_{\mathbf{G}}(\mathfrak{f})=\left\{g \in \mathbf{G} \mid \operatorname{Ad}_{\mathfrak{g}}(g)(\mathfrak{f})=\mathfrak{f}\right\} .
$$

The group $\mathbf{F}$ is connected and $F=\mathbf{G} / \mathbf{F}$ is a complex projective variety.

Let $\mathfrak{h}$ be a Cartan subalgebra of $\mathfrak{g}$, contained in $\mathfrak{f}$. Its elements are semisimple. Let $\mathfrak{h}_{\mathbb{R}}$ be the set of $H \in \mathfrak{h}$ for which $\operatorname{ad}(H)$ has real eigenvalues. Then $i \mathfrak{h}_{\mathbb{R}}$ is a maximal torus in $\mathfrak{g}$, and there is a compact form $\mathfrak{u}_{0}$ of $\mathfrak{g}$ containing $i \mathfrak{h}_{\mathbb{R}}$. The analytic subgroup $\mathbf{U}_{0}$ with $\operatorname{Lie}\left(\mathbf{U}_{0}\right)=\mathfrak{u}_{0}$ is a maximal compact subgroup of $\mathbf{G}$ and acts transitively on $F$. Let $\tau$ be the conjugation on $\mathfrak{g}$ defined by the real form $\mathfrak{u}_{0}$. There is a unique Levi-Chevalley decomposition

$$
\mathfrak{f}=\mathfrak{n}_{\mathfrak{g}}(\mathfrak{f}) \oplus \mathfrak{L}_{\tau}(\mathfrak{f}), \quad \mathfrak{L}_{\tau}(\mathfrak{f})=\mathfrak{z}_{\tau}(\mathfrak{f}) \oplus \mathfrak{s}_{\tau}(\mathfrak{f}),
$$

where $\mathfrak{n}_{\mathfrak{g}}(\mathfrak{f})$ is the ideal of the nilpotent elements of $\operatorname{rad}(\mathfrak{f})$, and coincides with its nilradical, and $\mathfrak{L}_{\tau}(\mathfrak{f})$ is its $\tau$-invariant reductive Levi factor, which further decomposes into the direct sum of its center $z_{\tau}(\mathfrak{f})$ and its semisimple ideal $\mathfrak{s}_{\tau}(\mathfrak{f})$. Let $A$ be 
any element of maximal rank in $\jmath_{\tau}(\mathfrak{f}) \cap \mathfrak{h}_{\mathbb{R}}$. Then the isotropy at the base point $p_{0}$ of the transitive action of $\mathbf{U}_{0}$ on $F$ is the group

$$
\mathbf{F}_{0}=\mathbf{F} \cap \mathbf{U}_{0}=\left\{g \in \mathbf{U}_{0} \mid \operatorname{Ad}_{\mathfrak{g}}(g)(A)=A\right\}=\mathrm{Z}_{\mathbf{U}_{0}}\left(\jmath_{\tau}(\mathfrak{f})\right) .
$$

We observe that $F$ can be viewed as an $\mathbf{U}_{0}$-homogeneous totally complex $C R$ manifold, with isotropy $\mathbf{F}_{0}$ and $C R$ algebra $\left(\mathfrak{u}_{0}, \mathfrak{f}\right)$ at the base point.

5.2. Orbits of a real form. Let $\mathbf{G}_{0}$ be a real form of $\mathbf{G}, \mathfrak{g}_{0}=\operatorname{Lie}\left(\mathbf{G}_{0}\right)$, and denote by $\sigma(X)=\bar{X}$ the conjugation in $\mathfrak{g}$ defined by $\mathfrak{g}_{0}$.

We consider the $\mathbf{G}_{0}$-orbit $E$ through the base point $p_{0}$ of $F=\mathbf{G} / \mathbf{F}$. It is a $\mathbf{G}_{0}$-homogeneous $C R$ manifold, having at the base point $p_{0}$ isotropy

$$
\mathbf{E}_{0}=\mathbf{F} \cap \mathbf{G}_{0}=\mathrm{N}_{\mathbf{G}_{0}}(\mathfrak{T})=\left\{g \in \mathbf{G}_{0} \mid \operatorname{Ad}_{\mathfrak{g}}(g)(\mathfrak{T})=\mathfrak{f}\right\} .
$$

and $C R$ algebra $\left(\mathfrak{g}_{0}, \mathfrak{f}\right)$. With this structure, $E$ is a generic $C R$-submanifold of $F$.

5.3. Mostow fibration. A pair $\left(\theta, \mathfrak{h}_{0}\right)$, consisting of a Cartan involution $\theta$ of $\mathfrak{g}_{0}$ and a $\theta$-invariant Cartan subalgebra $\mathfrak{h}_{0}$ of $\mathfrak{g}_{0}$ contained in $\mathfrak{f}$ was called in [5] adapted to $\left(\mathfrak{g}_{0}, \mathfrak{f}\right)$. If $\left(\theta, \mathfrak{h}_{0}\right)$ is adapted, the involutions $\theta$ and $\sigma$ commute and their composition $\tau=\sigma \circ \theta$ is the conjugation with respect to a compact form $\mathfrak{u}_{0}$ of $\mathfrak{g}$, for which we have (5.2).

The complexification $\mathfrak{h}$ of $\mathfrak{h}_{0}$ is a Cartan subalgebra of $\mathfrak{g}$ contained in $\mathfrak{f}$. Let $\mathcal{R} \subset \mathfrak{h}_{\mathbb{R}}^{*}$ be the root system of $(\mathfrak{g}, \mathfrak{h}), \mathfrak{g}^{\alpha}=\left\{X \in \mathfrak{g} \mid[H, X]=\alpha(H) X, \forall H \in \mathfrak{h}_{\mathbb{R}}\right\}$ the corresponding root spaces. There is an element $A \in z_{\tau}(\mathfrak{f}) \cap \mathfrak{b}_{\mathbb{R}}$ such that

$$
\mathfrak{n}_{\mathfrak{g}}(\mathfrak{f})=\sum_{\alpha(A)>0} \mathfrak{g}^{\alpha}, \quad \mathfrak{L}_{\tau}(\mathfrak{f})=\mathfrak{h} \oplus \sum_{\alpha(A)=0} \mathfrak{g}^{\alpha} .
$$

The set $\mathcal{F}=\{\alpha \in \mathcal{R} \mid \alpha(A) \geq 0\}=\left\{\alpha \in \mathcal{R} \mid \mathfrak{g}^{\alpha} \subset \mathfrak{f}\right\}$ is the parabolic set of $\mathfrak{f}$. It decomposes into the disjoint union $\mathcal{F}=\mathcal{F}_{n} \cup \mathcal{F}_{r}$, with $\mathcal{F}_{n}=\{\alpha \mid \alpha(A)>0\}$ and $\mathcal{F}_{r}=\{\alpha \mid \alpha(A)=0\}=\{\alpha \in \mathcal{F} \mid-\alpha \in \mathcal{F}\}$.

Consider the Cartan decomposition associated to $\theta$ :

$$
\mathfrak{g}_{0}=\kappa_{0} \oplus \mathfrak{p}_{0}, \quad \kappa_{0}=\left\{X \in \mathfrak{g}_{0} \mid \theta(X)=X\right\}, \quad \mathfrak{p}_{0}=\left\{X \in \mathfrak{g}_{0} \mid \theta(X)=-X\right\} .
$$

Then $\mathbf{K}_{0}=\mathbf{U}_{0} \cap \mathbf{G}_{0}$ has Lie algebra $\kappa_{0}$ and is a maximal compact subgroup of $\mathbf{G}_{0}$.

In [5, §10] we noticed that $E$ admits a Mostow fibration (see [20, 21]), whose basis $M$ is the $\mathbf{K}_{0}$ orbit through the base point $p_{0}$; it is a $\mathbf{K}_{0}$-homogeneous $C R$ manifold, with isotropy

$$
\mathbf{M}_{0}=\left\{g \in \mathbf{K}_{0} \mid \operatorname{Ad}_{\kappa}(g)(\mathfrak{f})=\mathfrak{f}\right\}=\left\{g \in \mathbf{K}_{0} \mid \operatorname{Ad}_{\kappa}(g)(A)=A\right\},
$$

and $C R$ algebra $\left(\kappa_{0}, \mathfrak{v}\right)$, with $\mathfrak{v}=\mathfrak{f} \cap \kappa$, at $p_{0}$.

We observe that $\mathbf{M}_{0}$ is maximally compact in $\mathbf{E}_{0}$, and is its deformation retract. In particular, $\mathbf{M}_{0}$ and $\mathbf{E}_{0}$ have the same number of connected components (see e.g. [5, §10]). We recall that $\mathbf{M}_{0}$ is connected when $M$ is a minimal orbit (see [3, §8]).

The $\mathbf{K}_{0}$-orbit $M$ in $F$ is the intersection of a $\mathbf{K}$-orbit $E^{*}$ with the $\mathbf{G}_{0}$-orbit $E$ in $F$ corresponding to $E^{*}$ in the Matsuki duality (cf. [15]). The $\mathbf{K}$-orbit $E^{*}$ provides a generic $C R$-embedding and a $\mathbf{K}$-realization of $M$. In general, the $C R$-embedding $M \hookrightarrow F$ is not generic, unless $E$ is the minimal orbit: indeed in this case $M=E$ and $E^{*}$ is open in $F$ (see [25, 3]). 
5.4. The base of the Mostow fibration. In this subsection we will show that $M$ is n-reductive, i.e. that $M$ is a minimal $\mathbf{K}_{0}$-orbit in $E^{*}$, and investigate some features of the algebra $\mathfrak{v}=\mathfrak{f} \cap \kappa$ which describes its $C R$ structure.

Having fixed an adapted $\left(\theta, \mathfrak{h}_{0}\right)$, we use (5.6) to split $\mathfrak{h}_{0}$ into its toroidal and vectorial parts:

$$
\mathfrak{h}_{0}=\mathfrak{h}_{0}^{+} \oplus \mathfrak{h}_{0}^{-}, \quad \text { with } \quad \mathfrak{h}_{0}^{+}=\mathfrak{h}_{0} \cap \mathfrak{\kappa}_{0}, \quad \mathfrak{h}_{0}^{-}=\mathfrak{h}_{0} \cap \mathfrak{p}_{0} .
$$

Then $\mathfrak{h}_{\mathbb{R}}=\mathfrak{h}_{0}^{-} \oplus i \mathfrak{h}_{0}^{+}$. The involutions $\sigma, \theta, \tau$ act on $\mathfrak{h}_{\mathbb{R}}$, yielding by duality involutions $\sigma^{*}, \theta^{*}, \tau^{*}$ on $\mathfrak{h}_{\mathbb{R}}^{*}$, which restrict to permutations of $\mathcal{R}$. Set $\sigma^{*}(\alpha)=\bar{\alpha}$. Then

$$
\begin{gathered}
\sigma^{*}(\alpha)=\bar{\alpha}, \quad \theta^{*}(\alpha)=-\bar{\alpha}, \quad \tau^{*}(\alpha)=-\alpha, \\
\sigma\left(\mathfrak{g}^{\alpha}\right)=\mathfrak{g}^{\bar{\alpha}}, \quad \theta\left(\mathfrak{g}^{\alpha}\right)=\mathfrak{g}^{-\bar{\alpha}}, \quad \tau\left(\mathfrak{g}^{\alpha}\right)=\mathfrak{g}^{-\alpha}, \quad \forall \alpha \in \mathcal{R} .
\end{gathered}
$$

We use the notation

$$
\mathcal{R}_{\mathrm{re}}=\{\alpha \in \mathcal{R} \mid \bar{\alpha}=\alpha\}, \quad \mathcal{R}_{\mathrm{im}}=\{\alpha \in \mathcal{R} \mid \bar{\alpha}=-\alpha\}, \quad \mathcal{R}_{\mathrm{cx}}=\{\alpha \in \mathcal{R} \mid \bar{\alpha} \neq \pm \alpha\},
$$

for the sets of real, imaginary and complex (meaning neither real nor imaginary) roots. Imaginary roots are further subdivided into compact and noncompact:

$$
\mathcal{R}_{\mathrm{im}}=\mathcal{R}_{\mathrm{cp}} \cup \mathcal{R}_{\mathrm{nc}}, \quad \text { with } \quad \mathcal{R}_{\mathrm{cp}}=\left\{\alpha \in \mathcal{R}_{\mathrm{im}} \mid \mathrm{g}^{\alpha} \subset \kappa\right\}, \quad \mathcal{R}_{\mathrm{nc}}=\left\{\alpha \in \mathcal{R}_{\mathrm{im}} \mid \mathrm{g}^{\alpha} \subset \mathfrak{p}\right\} .
$$

Let

$$
\pi: \mathfrak{g} \ni X \longrightarrow \frac{1}{2}(X+\theta(X)) \in \kappa .
$$

be the projection on $\kappa$ along $\mathfrak{p}$.

Lemma 5.1. There is a set $\mathcal{R}^{+}$of positive roots with the property that

$$
\bar{\alpha} \in \mathcal{R}^{+}, \forall \alpha \in \mathcal{R}^{+} \cap \mathcal{R}_{\mathrm{cx}},
$$

yielding a direct sum decomposition of $\kappa$ :

$$
\kappa=\mathfrak{h}^{+} \oplus \sum_{\alpha \in \mathcal{R}^{+} \cap \mathcal{R}_{\mathrm{cp}}} \mathfrak{g}^{-\alpha} \oplus \sum_{\alpha \in \mathcal{R}^{+} \backslash \mathcal{R}_{\mathrm{nc}}} \pi\left(\mathfrak{g}^{\alpha}\right) .
$$

Proof. The existence of a set of positive roots satisfying (5.10) is, e.g., a consequence of [5, Lemma 4.3]. Then (5.11) follows because $\pi\left(\mathfrak{g}^{\alpha}\right)=\pi\left(\mathfrak{g}^{-\bar{\alpha}}\right)$ for all $\alpha \in \mathcal{R}$ and $\pi\left(\mathfrak{g}^{\alpha}\right)=\{0\}$ for $\alpha \in \mathcal{R}_{\mathrm{nc}}$.

Lemma 5.2. Let $\mathcal{F}$ be the parabolic set of $\mathfrak{f}$ and $\mathcal{F}^{*}=\mathcal{F} \backslash \mathcal{R}_{\mathrm{nc}}$. Then

$$
\mathfrak{v}=\mathfrak{f} \cap \kappa=\mathfrak{h}^{+} \oplus \sum_{\alpha \in \mathcal{F} \theta} \pi\left(\mathfrak{g}^{\alpha}\right) \text {, with } \mathcal{F}^{\theta}=\left\{\alpha \in \mathcal{F}^{*} \mid-\bar{\alpha} \in \mathcal{F}\right\}=\mathcal{F}^{*} \cap \theta^{*}\left(\mathcal{F}^{*}\right) \text {. }
$$

Proof. An $X \in \mathfrak{f}$ uniquely decomposes as

$$
X=H+\sum_{\alpha \in \mathcal{F}} X_{\alpha}, \quad \text { with } H \in \mathfrak{h} \text { and } X_{\alpha} \in \mathfrak{g}^{\alpha} .
$$

If $\theta(X)=X$, from

we obtain

$$
X=\theta(X)=\theta(H)+\sum_{\alpha \in \mathcal{F}} \theta\left(X_{\alpha}\right)
$$

This yields (5.12).

Split $\mathcal{F}^{\theta}$ into the two subsets

$$
\mathcal{F}_{n}^{\theta}=\mathcal{F}^{\theta} \cap \mathcal{F}_{n}, \quad \mathcal{F}_{r}^{\theta}=\mathcal{F}_{r} \cap \theta^{*}\left(\mathcal{F}_{r}\right)=\left\{\alpha \in \mathcal{F}_{r} \mid-\bar{\alpha} \in \mathcal{F}_{r}\right\} .
$$

Lemma 5.3. $\mathcal{F}^{\theta}, \mathcal{F}_{r}^{\theta}, \mathcal{F}_{n}^{\theta}$ are closed set of roots. 
Lemma 5.4. Let $\mathfrak{n}_{\mathfrak{g}}(\mathfrak{f})$ be the ideal of the nilpotent elements of the radical of $\mathfrak{f}$. Then

$$
\mathfrak{n}_{\kappa}(\mathfrak{p})=\left\{X+\theta(X) \mid X \in \mathfrak{n}_{\mathfrak{g}}(\mathfrak{f}), \theta(X) \in \mathfrak{f}\right\}=\sum_{\alpha \in \mathcal{F}_{n}^{\theta^{*}}} \pi\left(\mathfrak{g}^{\alpha}\right) .
$$

Proof. Set $\mathfrak{y}=\left\{X+\theta(X) \mid X \in \mathfrak{n}_{\mathfrak{g}}(\mathfrak{f}), \theta(X) \in \mathfrak{f}\right\}$. First we prove that $\mathfrak{y}$ is an ideal in v. In fact, we have

$$
[X+\theta(X), Y+\theta(Y)]=[X, Y]+\theta([X, Y])+[X, \theta(Y)]+\theta([X, \theta(Y)]),
$$

with $[X, Y]$ and $[X, \theta(Y)]$ both belonging to $\mathfrak{n}_{\mathrm{g}}(\mathfrak{f})$.

If $X+\theta(X)$ is an element of $\mathfrak{y}$, then $[X, \theta(X)] \in \mathfrak{n}_{\mathfrak{g}}(\mathfrak{f})$. We need to prove that also $X+\theta(X)$ is nilpotent. To this aim, we observe that $(\operatorname{ad}(X+\theta(X)))^{m}(Y)$ is a sum of terms of the form $\left[Z_{1}, \ldots, Z_{m}, Y\right]$ with $Z_{i} \in\{X, \theta(X)\}$ for $i=1, \ldots, m$.

Since $\mathfrak{n}_{\mathfrak{g}}(\mathfrak{f})$ is an algebra of nilpotent elements of $\mathfrak{g}$, there is some integer $v$ such that $\left[X_{1}, \ldots, X_{h}, Y\right]=0$ for all $Y \in \mathfrak{g}$ if $X_{1}, \ldots, X_{h} \in \mathfrak{n}_{\mathfrak{g}}(\mathfrak{f})$ and $h \geq v$. We claim that

(*) $\quad\left[X_{1}, \ldots, X_{m}, Y\right]=0, \forall Y \in \mathfrak{g}$ if $X_{1}, \ldots, X_{m} \in \mathfrak{f}$ and $\#\left\{i \mid X_{i} \in \mathfrak{n}_{\mathfrak{g}}(\mathfrak{f})\right\} \geq v$.

To prove our claim, we argue by recurrence on $m$. In fact, when $m=v$, (因) is clearly satisfied. Assume now that $m>v$ and that $(\mathbb{*})$ is true for commutators of a lesser number of elements. Clearly $\left[X_{1}, \ldots, X_{m}, Y\right]=0$ when all $X_{i}$ 's belong to $\mathfrak{n}_{\mathfrak{g}}(\mathfrak{f})$. Take now $X_{1}, \ldots, X_{m} \in \mathfrak{f}$ with at least $v$ elements in $\mathfrak{n}_{\mathfrak{g}}(\mathfrak{f})$ and at least one, say $X_{i_{0}}$, in $\mathfrak{f} \backslash \mathfrak{n}_{\mathfrak{g}}(\mathfrak{f})$. The commutator $\left[X_{1}, \ldots, X_{m}, Y\right]$ is a linear combination of the commutators $\left[X_{1}, \ldots, X_{i_{0}-1}, X_{i_{0}+1}, \ldots,\left[X_{i_{0}}, Y\right]\right]$ and $\left[X_{1}, \ldots,\left[X_{i_{0}}, X_{j}\right], \ldots, Y\right]$. All these terms are zero by the recursive assumption.

This shows that the terms of the form $\left[Z_{1}, \ldots, Z_{m}, Y\right]$ with at least $v$ of the $Z_{i}^{\prime} s$ equal to $X$ are zero for any choice of $Y$.

Using the fact that $\theta$ is an automorphism of $\mathfrak{g}$, it follows that also the terms $\left[Z_{1}, \ldots, Z_{m}, Y\right]$ for which at least $v$ elements equal $\theta(X)$ are zero, showing that $(\operatorname{ad}(X+\theta(X)))^{2 v-1}=0$.

This proves that $\mathfrak{y}$ is an ideal of nilpotent elements in $\mathfrak{v}$. Hence, $\mathfrak{y} \subset \mathfrak{n}_{\mathfrak{K}}(\mathfrak{y})$. From

$$
\mathfrak{y}=\sum_{\alpha \in \mathcal{F}_{n}^{\theta}} \pi\left(\mathfrak{g}^{\alpha}\right)
$$

and the decomposition (5.12) of $\mathfrak{v}$ in Lemma5.2 we obtain that

$$
\mathfrak{L}(\mathfrak{p})=\mathfrak{h}^{+} \oplus \sum_{\alpha \in \mathcal{F}_{r}^{\theta}} \pi\left(\mathfrak{g}^{\alpha}\right)=\mathfrak{v} \cap \overline{\mathfrak{v}}
$$

is a reductive complement of $\mathfrak{y}$ in $\mathfrak{v}$. Hence $\mathfrak{y}=\mathfrak{n}_{\mathfrak{K}}(\mathfrak{y})$.

We summarize the results obtained so far in this section by the following

Theorem 5.5. Let $\left(\mathfrak{g}_{0}, \mathfrak{f}\right)$ be a CR algebra with $\mathfrak{g}_{0}$ semisimple and $\mathfrak{f}$ parabolic in the complexification $\mathfrak{g}$ of $\mathfrak{g}_{0},\left(\theta, \mathfrak{h}_{0}\right)$ an adapted pair, $\kappa_{0}=\left\{X \in \mathfrak{g}_{0} \mid \theta(X)=X\right\}$ the corresponding maximal compact subalgebra of $\mathrm{g}_{0}, \kappa$ its complexification and $\mathfrak{v}=\mathfrak{f} \cap \kappa$. Then $\mathfrak{v} \cap \overline{\mathfrak{v}}$ is a reductive complement of $\mathfrak{n}_{\kappa}(\mathfrak{v})$ in $\mathfrak{v}$.

Hence, the $\mathbf{K}_{0}$-orbit $M$ of $\mathfrak{f}$ in $\mathfrak{g}$ is an $\mathfrak{n}$-reductive CR manifold.

We also have

Proposition 5.6. The closed Lie group $\mathbf{V}=\mathbf{F} \cap \mathbf{K}$ admits a Levi-Chevalley decomposition

$$
\mathbf{V}=\mathbf{V}_{n} \mathbf{V}_{r}, \text { with } \operatorname{Lie}\left(\mathbf{V}_{n}\right)=\mathfrak{n}_{\kappa}(\mathfrak{p}), \quad \operatorname{Lie}\left(\mathbf{V}_{r}\right)=\mathfrak{L}(\mathfrak{v})=\mathfrak{m} .
$$


Proof. Indeed $\mathbf{G}$ is an algebraic group and $\mathbf{V}$ is an algebraic subgroup of $\mathbf{G}$, hence admitting a Levi-Chevalley decomposition (5.17) (see e.g. [22, Ch.6]).

Example 5.7. Consider the minimal orbit $M$ of $\mathbf{S U}(2,3)$ in the complex flag manifold of lines and 3-planes of $\mathbb{C}^{5}$ :

$$
M^{(2,6)}=\left\{\text { flags }\left(L_{1} \subset L_{3}\right) \text { with } L_{1} \subset L_{3}^{\perp} \subset L_{3}\right\},
$$

where the perpendicularity is taken with respect to a Hermitian symmetric form $h$ of signature $(2,3)$ in $\mathbb{C}^{5}$.

This is the minimal orbit described by the cross marked Satake diagram

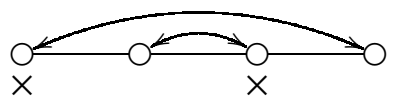

Let $\mathrm{h}$ be defined by the anti-diagonal matrix $\left({ }_{1} 1^{1}{ }^{1}\right)$.

We give below a matrix description of the complexification $\kappa$ of the maximal compact subalgebra $\kappa_{0}=\mathfrak{s}\left(\mathfrak{u}_{2} \times \mathfrak{H}_{3}\right)$ of $\mathfrak{s u}_{2,3}$, of $\mathfrak{v}=\mathfrak{f} \cap \kappa$, and of its normalizer in $\kappa$ :

$$
\kappa:\left(\begin{array}{ccccc}
\lambda_{2} & \zeta_{1} & \eta_{1} & \eta_{2} & \mu_{2} \\
z_{1} & \lambda_{1} & z_{2} & \mu_{1} & w_{2} \\
w_{1} & \zeta_{2} & \lambda_{0} & \zeta_{2} & w_{1} \\
w_{2} & \mu_{1} & z_{2} & \lambda_{1} & z_{1} \\
\mu_{2} & \eta_{2} & \eta_{1} & \zeta_{1} & \lambda_{2}
\end{array}\right), \quad \mathfrak{v}=\kappa \cap \mathfrak{f}:\left(\begin{array}{ccccc}
\lambda_{2} & \zeta_{1} & 0 & 0 & 0 \\
0 & \lambda_{1} & 0 & 0 & 0 \\
0 & \zeta_{2} & \lambda_{0} & \zeta_{2} & 0 \\
0 & 0 & 0 & \lambda_{1} & 0 \\
0 & 0 & 0 & \zeta_{1} & \lambda_{2}
\end{array}\right) .
$$

The normalizer

$$
\mathrm{N}_{\kappa}(\kappa \cap \mathfrak{f}):\left(\begin{array}{ccccc}
\lambda_{2} & \zeta_{1} & 0 & 0 & \mu \\
0 & \lambda_{1} & 0 & \mu & 0 \\
0 & \zeta_{2} & \lambda_{0} & \zeta_{2} & 0 \\
0 & \mu & 0 & \lambda_{1} & 0 \\
\mu & 0 & 0 & \zeta_{1} & \lambda_{2}
\end{array}\right)
$$

of $\kappa \cap \mathfrak{f}$ does not contain any maximal torus in $\kappa$, and hence $\mathfrak{v}$ is not regular. This example shows that we cannot expect, in general, that the basis $M$ of the Mostow fibration of a $\mathbf{G}_{0}$-orbit has a $\mathrm{t}_{0}$-regular $\mathfrak{p}$.

We have indeed

Proposition 5.8. Let $\mathfrak{v}=\mathfrak{f} \cap \kappa$ and set $\mathfrak{L}(\mathfrak{v})=\mathfrak{v} \cap \overline{\mathfrak{v}}$. Then the following are equivalent:

(1) $\left(\kappa_{0}, \mathfrak{v}\right)$ is of type $\mathbb{I}$, i.e. $\mathfrak{Q}(\mathfrak{v})$ is normalized by a maximal torus $\mathrm{t}_{0}$ of $\kappa_{0}$;

(2) there exists a maximal set of strongly orthogonal real roots $\left\{\alpha_{1}, \ldots, \alpha_{r}\right\}$ in $\mathcal{F}$ such that

$$
\beta \in \mathcal{F}_{r}^{\theta}, \quad 1 \leq i \leq r, \beta+\alpha_{i} \in \mathcal{R} \Longrightarrow \beta+\alpha_{i} \in \mathcal{F}_{r}^{\theta} .
$$

(3) if $\left(\theta, \mathfrak{h}_{0}\right)$ is an adapted pair with $\mathfrak{h}_{0} \subset \mathfrak{f}$ maximally compact, then there exists a maximal system of strongly orthogonal real roots $\left\{\alpha_{1}, \ldots, \alpha_{r}\right\}$ in $\mathcal{F}_{n}$ such that

$$
\beta+\alpha_{i} \notin \mathcal{R}, \quad \forall \beta \in \mathcal{F}_{r}^{\theta}, 1 \leq i \leq r .
$$

The following are equivalent

(a) $\left(\kappa_{0}, \mathfrak{v}\right)$ is of type $\mathrm{I}$, i.e. $\mathfrak{v}$ is normalized by a maximal torus $\mathrm{t}_{0}$ of $\kappa_{0}$; 
(b) there is a maximal system of strongly orthogonal real roots $\left\{\alpha_{1}, \ldots, \alpha_{r}\right\}$ in $\mathcal{F}$ such that

$$
1 \leq i \leq r, \quad \beta \in \mathcal{F}^{\theta}, \quad \alpha_{i}+\beta \in \mathcal{R} \Longrightarrow \alpha_{i}+\beta \in \mathcal{F}^{\theta} ;
$$

(c) if $\left(\theta, \mathfrak{h}_{0}\right)$ is an adapted pair with $\mathfrak{h}_{0} \subset \mathfrak{f}$ maximally compact, then there exists a maximal system of strongly orthogonal real roots $\left\{\alpha_{1}, \ldots, \alpha_{r}\right\}$ in $\mathcal{F}_{n}$ such that

$$
1 \leq i \leq r, \beta \in \mathcal{F}^{\theta}, \quad \alpha_{i}+\beta \in \mathcal{R} \Longrightarrow \alpha_{i}+\beta \in \mathcal{F}^{\theta} .
$$

In particular, $\mathfrak{v}$ is regular in $\kappa$ when $\mathfrak{f} \cap \mathfrak{g}_{0}$ contains a maximal torus of $\mathfrak{g}_{0}$.

Proof. Fix a maximally compact Cartan subalgebra $\mathfrak{h}_{0}$ adapted to $\left(\mathfrak{g}_{0}, \mathfrak{f}\right)$. Then $\mathcal{F}_{r}$ does not contain any real root and every $\theta$-invariant maximal torus of $\mathfrak{g}_{0}$ can be obtained by applying to $\mathfrak{h}_{0}$ the Cayley transform with respect to a maximal set $\left\{\alpha_{1}, \ldots, \alpha_{r}\right\}$ of strongly orthogonal real roots contained in $\mathcal{F}_{n}$ (see [13]). If $\beta \in \mathcal{F}$ and $\beta+\alpha_{i} \in \mathcal{R}$, then $\beta+\alpha_{i} \in \mathcal{F}_{n}$. This gives the equivalence (1) $\Leftrightarrow(3)$. We prove the equivalence (2) $\Leftrightarrow(3)$ using the Cayley transform with respect to a maximal system of strongly orthogonal real roots in $\mathcal{F}_{r}$.

Assume that (c) holds true. We want to prove that we have also (3). Indeed, assume by contradiction that there are $\beta \in \mathcal{F}^{\theta}$ and $1 \leq i \leq r$, with $\alpha_{i}+\beta \in \mathcal{R}$. Since $\pm \beta, \pm \bar{\beta} \in \mathcal{F}$, we have $\alpha_{1}+\beta, \alpha_{i}+\bar{\beta} \in \mathcal{F}_{n}$. By the assumption that $\alpha_{1}+\beta, \alpha_{i}+\bar{\beta} \in \mathcal{F}^{\theta}$ we get a contradiction, because also $\theta^{*}\left(\alpha_{1}+\beta\right)=-\alpha_{1}-\bar{\beta}, \theta^{*}\left(\alpha_{i}+\bar{\beta}\right)=-\alpha_{i}-\beta \in \mathcal{F}$, and this gives in particular $\alpha_{i}+\beta \in \mathcal{F}_{r}$.

The equivalence $(a) \Leftrightarrow(b) \Leftrightarrow(c)$ is then proved with arguments similar to those used to show that (1) $\Leftrightarrow(2) \Leftrightarrow(3)$.

In Example 5.7 denoting by $\alpha_{1}, \ldots, \alpha_{4}$ the simple roots in the Satake diagram, we have

$$
\begin{gathered}
\mathcal{F}_{n}=\left\{\alpha_{1}, \alpha_{1}+\alpha_{2}, \alpha_{1}+\alpha_{2}+\alpha_{3}, \alpha_{1}+\alpha_{2}+\alpha_{3}+\alpha_{4}, \alpha_{2}+\alpha_{3}, \alpha_{2}+\alpha_{3}+\alpha_{4}, \alpha_{3}, \alpha_{3}+\alpha_{4}\right\}, \\
\mathcal{F}_{r}=\left\{ \pm \alpha_{2}, \pm \alpha_{4}\right\}, \mathcal{F}_{n}^{\theta}=\left\{\alpha_{1}, \alpha_{3}\right\}, \mathcal{F}_{r}^{\theta}=\emptyset, \mathcal{F}_{n} \cap \mathcal{R}_{\mathrm{re}}=\left\{\alpha_{2}+\alpha_{3}, \alpha_{1}+\alpha_{2}+\alpha_{3}+\alpha_{4}\right\}, \\
\alpha_{1}+\left(\alpha_{2}+\alpha_{3}\right) \in \mathcal{R}, \quad-\overline{\alpha_{1}+\alpha_{2}+\alpha_{3}}=-\left(\alpha_{2}+\alpha_{3}+\alpha_{4}\right) \notin \mathcal{F} \Rightarrow \alpha_{1}+\alpha_{2}+\alpha_{3} \notin \mathcal{F}^{\theta} .
\end{gathered}
$$

Since $\mathcal{F}_{n} \cap \mathcal{R}_{\mathrm{re}}$ is the unique maximal system of strongly orthogonal real roots in $\mathcal{F}$, this shows that $\mathfrak{v}$ does not satisfy (b).

Example 5.9. Let $M$ be the minimal orbit of $\mathbf{S O}(3,5)$ in the flag $F$ of the 3 -planes contained in the complex quadric of $\mathbb{C P}^{7}$, associated to the cross-marked Satake diagram

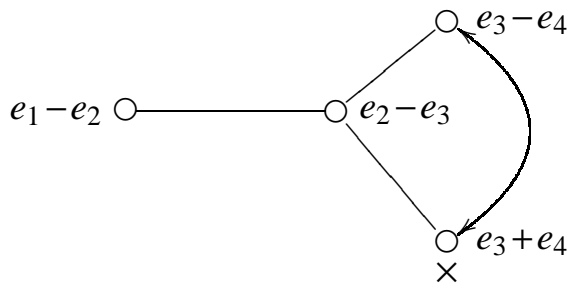

We have $\mathcal{F}=\mathcal{F}_{n} \cup \mathcal{F}_{r}$ and $\mathcal{F}^{\theta}=\mathcal{F}_{n}^{\theta} \cup \mathcal{F}_{r}^{\theta}$ with

$$
\begin{aligned}
\mathcal{F}_{n} & =\left\{e_{i}+e_{j} \mid 1 \leq i<j \leq 4\right\}, & \mathcal{F}_{r} & =\left\{ \pm\left(e_{i}-e_{j}\right) \mid 1 \leq i<j \leq 4\right\}, \\
\mathcal{F}_{n}^{\theta} & =\left\{e_{i}+e_{4} \mid 1 \leq i \leq 3\right\}, & \mathcal{F}_{r}^{\theta} & =\left\{ \pm\left(e_{i}-e_{j}\right) \mid 1 \leq i<j \leq 3\right\} .
\end{aligned}
$$


The maximal sets of strongly orthogonal real roots are the sets $E_{i, j}=\left\{e_{i} \pm e_{j}\right\}$ for $1 \leq i<j \leq 3$. Then condition (2) is not satisfied and $\mathfrak{L}(\mathfrak{v})$ is not $\mathrm{t}_{0}$-regular for any maximal torus $\mathrm{t}_{0}$ in $\kappa_{0} \simeq\left(\mathfrak{s D}_{3} \oplus \mathfrak{s D}_{5}\right)$.

\section{Parabolic Regularization}

In this section, $\kappa$ is a reductive complex Lie algebra. Starting from any complex Lie subalgebra $\mathfrak{v}$ of $\kappa$, we describe a canonical regularization procedure to associate to $\mathfrak{v}$ a suitable parabolic subalgebra $\mathfrak{w}$ of $\kappa$.

Lemma 6.1. Let $\mathfrak{v}$ be a Lie subalgebra of a reductive Lie algebra $\kappa$. Define by recurrence

$$
\left\{\begin{array}{l}
\mathfrak{v}_{0}=\mathfrak{v}, \\
\mathfrak{v}_{m+1}=\mathrm{N}_{\kappa}\left(\mathfrak{n}_{\kappa}\left(\mathfrak{v}_{m}\right)\right) \quad \text { for } m \geq 0,
\end{array}\right.
$$

where $\mathrm{N}_{\kappa}\left(\mathfrak{n}_{\kappa}\left(\mathfrak{v}_{m}\right)\right)=\left\{X \in \kappa \mid\left[X, \mathfrak{n}_{\kappa}\left(\mathfrak{v}_{m}\right)\right] \subset \mathfrak{n}_{\kappa}\left(\mathfrak{v}_{m}\right)\right\}$ is the normalizer of $\mathfrak{n}_{\kappa}\left(\mathfrak{v}_{m}\right)$ in $\kappa$. Then we obtain two non decreasing sequences of Lie subalgebras

$$
\begin{gathered}
\mathfrak{n}_{\kappa}(\mathfrak{y})=\mathfrak{n}_{\kappa}\left(\mathfrak{v}_{0}\right) \subset \mathfrak{n}_{\kappa}\left(\mathfrak{p}_{1}\right) \subset \cdots \subset \mathfrak{n}_{\kappa}\left(\mathfrak{v}_{m}\right) \subset \mathfrak{n}_{\kappa}\left(\mathfrak{p}_{m+1}\right) \subset \cdots, \\
\mathfrak{v}=\mathfrak{v}_{0} \subset \mathfrak{v}_{1} \subset \cdots \subset \mathfrak{v}_{m} \subset \mathfrak{v}_{m+1} \subset \cdots
\end{gathered}
$$

Proof. Since each $\mathfrak{n}_{\kappa}\left(\mathfrak{v}_{m}\right)$ is an ideal in $\mathfrak{v}_{m}$, from $\left[\mathfrak{v}_{m}, \mathfrak{n}_{\kappa}\left(\mathfrak{v}_{m}\right)\right] \subset \mathfrak{n}_{\kappa}\left(\mathfrak{v}_{m}\right)$ we have $\mathfrak{v}_{m} \subset \mathfrak{v}_{m+1}$.

Moreover, $\mathfrak{n}_{\kappa}\left(\mathfrak{v}_{m}\right)$, being a nilpotent ideal of $\mathfrak{v}_{m+1}$, is contained in $\operatorname{rad}\left(\mathfrak{v}_{m+1}\right)$. It consists of nilpotent elements and therefore $\mathfrak{n}_{\kappa}\left(\mathfrak{v}_{m}\right) \subset \mathfrak{n}_{\kappa}\left(\mathfrak{p}_{m+1}\right)$.

We have (see [24, 7, 12]):

Proposition 6.2. The sequences (6.2) and (6.3) stabilize and

$$
\mathfrak{n}_{\kappa}\left(\mathfrak{v}_{m}\right)=\mathfrak{n}_{\kappa}\left(\mathfrak{v}_{m+1}\right) \quad \Longrightarrow \quad \mathfrak{v}_{m+1}=\mathfrak{v}_{h+1} \text { and } \mathfrak{n}_{\kappa}\left(\mathfrak{v}_{m}\right)=\mathfrak{n}_{\kappa}\left(\mathfrak{w}_{h}\right), \quad \forall h \geq m .
$$

The Lie subalgebra $\mathfrak{w}=\bigcup_{m} \mathfrak{v}_{m}$ is parabolic in $\kappa$.

Proof. The first assertions follows because (6.2) and (6.3) are increasing sequences of linear subspaces of a finite dimensional vector space.

Assume that $\mathfrak{w}=\mathfrak{v}_{h}$ for all $h \geq m$. Since $\mathfrak{n}_{\mathfrak{K}}(\mathfrak{w})$ is a characteristic ideal of $\mathfrak{w}$, we have

$$
\mathfrak{w} \subset \mathrm{N}_{\kappa}(\mathfrak{w}) \subset \mathrm{N}_{\kappa}\left(\mathfrak{n}_{\kappa}(\mathfrak{w})\right)=\mathfrak{w} .
$$

Hence $\mathfrak{w}$ is its own normalizer and equals the normalizer of $n_{\kappa}(\mathfrak{w})$. Then the statement follows from Lemma 6.3 below.

We have (see e.g. [19, 24, 23, 7, 12])

Lemma 6.3. Let $\mathfrak{w}$ be a Lie subalgebra of $\kappa$, and assume that, for $\mathfrak{n}=\mathfrak{n}_{\kappa}(\mathfrak{w})$,

$$
\mathfrak{w}=\mathrm{N}_{\kappa}(\mathfrak{w})=\{X \in \kappa \mid[X, \mathfrak{w}] \subset \mathfrak{w}\}=\mathrm{N}_{\kappa}(\mathfrak{n})=\{X \in \kappa \mid[X, \mathfrak{n}] \subset \mathfrak{n}\} .
$$

Then $\mathfrak{w}$ is parabolic in $\kappa$.

The proof of Lemma 6.3 utilizes the following lemma.

Lemma 6.4. Let $\mathrm{w}$ be a complex Lie subalgebra of $\mathrm{\kappa}$ and $\mathrm{r}$ any solvable subalgebra of $\mathfrak{w}$. Then there exists an inner automorphism $\psi$ of $\mathfrak{w}$ such that

$$
\mathfrak{n}_{\kappa}(\mathfrak{r} \cap \psi(\mathfrak{r})) \subset \mathfrak{n}_{\kappa}(\mathfrak{w}) .
$$


Proof. First assume that $\mathfrak{w}$ is reductive. Then, taking any Borel subalgebra $\mathfrak{b}$ of $\mathfrak{w}$ containing $\mathfrak{r}$, we obtain the statement as a consequence of the existence in $\mathfrak{w}$ of a Borel subalgebra opposite to $\mathfrak{b}$ (see e.g. [8, 10]).

In general, we note that the quotient $\mathfrak{w} / n_{\kappa}(\mathfrak{w})$ is reductive and apply the previous argument to its solvable subalgebra $\left(\mathfrak{r}+\mathfrak{n}_{\kappa}(\mathfrak{w})\right) / \mathfrak{n}_{\kappa}(\mathfrak{w})$.

Proof of Lemma 6.3 Let $\mathfrak{b}, \mathfrak{b}^{\prime}$ be two Borel subalgebras of $\kappa$ with the two properties

$-\mathfrak{b} \cap \mathfrak{w}$ and $\mathfrak{b}^{\prime} \cap \mathfrak{w}$ are Borel subalgebras of $\mathfrak{w}$;

- $\mathfrak{n}_{\kappa}\left(\mathfrak{b} \cap \mathfrak{b}^{\prime} \cap \mathfrak{w}\right) \subset \mathfrak{n}$.

The existence of such a pair $\left(\mathfrak{b}, \mathfrak{b}^{\prime}\right)$ is granted by Lemma6.4 Indeed, any Borel subalgebra $\mathfrak{r}$ of $\mathfrak{w}$ is the intersection of $\mathfrak{w}$ with a Borel subalgebra $\mathfrak{b}$ of $\kappa$, and the inner automorphism $\psi$ of $\mathfrak{w}$ extends to an inner automorphism of $\kappa$, so that $\psi(\mathfrak{r})=\psi(\mathfrak{b}) \cap \mathfrak{w}$.

Since all Borel subalgebras of $\mathfrak{w}$ contain $\mathfrak{n}$, we actually have $\mathfrak{n}_{\kappa}\left(\mathfrak{b} \cap \mathfrak{b}^{\prime} \cap \mathfrak{w}\right)=\mathfrak{n}$. We claim that, in fact, $\mathfrak{n}_{\kappa}\left(\mathfrak{b} \cap \mathfrak{b}^{\prime}\right)=\mathfrak{n}$. Indeed, $N_{\mathfrak{n}_{\kappa}\left(\mathfrak{b} \cap \mathfrak{b}^{\prime}\right)}(\mathfrak{n}) \subset \mathfrak{w}$ and hence

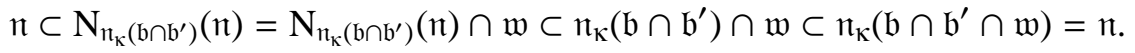

We obtain that $\mathfrak{n}_{\kappa}\left(\mathfrak{b} \cap \mathfrak{b}^{\prime}\right)=\mathfrak{n}$, because otherwise we would have $\mathfrak{n} \varsubsetneqq N_{\mathfrak{n}_{\kappa}\left(\mathfrak{b} \cap \mathfrak{b}^{\prime}\right)}(\mathfrak{n})$. It follows that $\mathfrak{b} \cap \mathfrak{b}^{\prime} \subset \mathfrak{w}$ and, in particular, that $\mathfrak{w}$ contains a Cartan subalgebra $t$ of $\kappa$. Let $\mathcal{K}$ be the root system of $(\kappa, \mathrm{t})$, set $\mathcal{K}^{+}=\left\{\alpha \in \mathcal{K} \mid \kappa^{\alpha} \subset \mathfrak{b}\right\}$, and denote by $\mathcal{B}$ the set of simple roots of $\mathcal{K}^{+}$. To prove that $\mathfrak{w}$ is parabolic, it suffices to show that $\kappa^{\beta} \subset \mathfrak{w}$ for all $\beta \in \mathcal{B}$, since this implies that $\mathfrak{b} \subset \mathfrak{w}$. Assume by contradiction that $\kappa^{\beta} \not \subset \mathfrak{w}$ for some $\beta \in \mathcal{B}$. Then $\kappa^{\beta} \not \subset \mathfrak{b}^{\prime}$. Hence $\kappa^{-\beta} \subset \mathfrak{b}^{\prime}$. Consider the Borel subalgebra

$$
\mathfrak{b}^{\prime \prime}=\mathrm{t} \oplus \mathrm{\kappa}^{-\beta} \oplus \sum_{\beta \in \mathcal{K}^{+} \backslash\{\beta\}} \kappa^{\alpha} .
$$

We have $\mathfrak{b} \cap \mathfrak{w} \subset \mathfrak{b}^{\prime \prime} \cap \mathfrak{w}$ and the two sets are equal because $\mathfrak{b} \cap \mathfrak{w}$ is maximal solvable in $\mathfrak{w}$. Then $\mathfrak{b}^{\prime}, \mathfrak{b}^{\prime \prime}$ are Borel subalgebras of $\kappa$ whose intersections with $\mathfrak{w}$ are Borel subalgebras of $\mathfrak{w}$, and

$$
\mathfrak{n}_{\kappa}\left(\mathfrak{b}^{\prime} \cap \mathfrak{b}^{\prime \prime} \cap \mathfrak{w}\right)=\mathfrak{n}_{\kappa}\left(\mathfrak{b}^{\prime} \cap \mathfrak{b} \cap \mathfrak{w}\right) \subset \mathfrak{n} .
$$

This implies, by the previous argument, that $\kappa^{-\beta} \subset \mathfrak{b}^{\prime} \cap \mathfrak{b}^{\prime \prime} \subset \mathfrak{w}$. But this in turns brings $\kappa^{-\beta} \subset \mathfrak{n}$, contradicting the inclusion $\mathfrak{n} \subset \mathfrak{b}$. The proof is complete.

Definition 6.5. We call the Lie subalgebra $\mathfrak{w}$ of Proposition 6.2 the parabolic regularization of $\mathfrak{v}$ in $\kappa$.

\section{THE DEPLOYMENT THEOREM}

Let $\mathbf{K}_{0}$ be a connected compact Lie group, $M$ a $\mathbf{K}_{0}$-homogeneous $C R$ manifold, with isotropy subgroup $\mathbf{M}_{0}$ and $C R$ algebra $\left(\kappa_{0}, \mathfrak{v}\right)$ at a point $p_{0} \in M$. We set $\kappa_{0}=\operatorname{Lie}\left(\mathbf{K}_{0}\right), \kappa=\mathbb{C} \otimes_{\mathbb{R}} \kappa_{0}, \mathfrak{m}_{0}=\operatorname{Lie}\left(\mathbf{M}_{0}\right), \mathfrak{m}=\mathbb{C} \otimes_{\mathbb{R}} \mathfrak{m}_{0}$. Note that $\mathfrak{m} \subset \mathfrak{v} \subset \kappa$.

Let $\mathbb{P}_{\kappa}$ be the set of all parabolic subalgebras of $\kappa$. Recall that, for $q \in \mathbb{P}_{\kappa}$, the intersection $\mathfrak{L}(\mathfrak{q})=\mathfrak{q} \cap \overline{\mathfrak{q}}$ is the reductive Levi factor of a Levi-Chevalley decomposition of $\mathfrak{q}$ (see Lemma4.11). For $\mathfrak{q} \in \mathbb{P}_{\kappa}$, we set

$$
\mathbf{N}_{\mathfrak{q}}=\left\{g \in \mathbf{K}_{0} \mid \operatorname{Ad}(g)(\mathfrak{q})=\mathfrak{q}\right\}, \quad N_{\mathfrak{q}}=\mathbf{K}_{0} / \mathbf{N}_{\mathfrak{q}} .
$$

With the $\mathbf{K}_{0}$-equivariant $C R$ structure $\left(\kappa_{0}, \mathfrak{q}\right)$ at the base point, $N_{\mathfrak{q}}$ is a complex flag manifold of $\kappa$. Its complex structure only depends on the pair $(\kappa, q)$ (see e.g. [25]). 
Moreover, $N_{\mathrm{q}}$ is simply connected and the isotropy subgroup $\mathbf{N}_{\mathrm{q}}$ is the centralizer of a torus of $\kappa_{0}$. Set

$$
\mathfrak{s}(\mathfrak{q})=[\mathfrak{Q}(\mathfrak{q}), \mathfrak{L}(\mathfrak{q})], \quad \mathfrak{z}(\mathfrak{q})=\mathbb{Z}(\mathfrak{L}(\mathfrak{q}))=\{X \in \mathfrak{L}(\mathfrak{q}) \mid[X, \mathfrak{L}(\mathfrak{q})]=\{0\}\}
$$

for the semisimple ideal and the center of its canonical reductive factor $\mathfrak{L}(\mathfrak{q})$. Then $\mathbf{N}_{\mathfrak{q}}$ is the centralizer of the torus $\jmath_{0}(\mathfrak{q})=\mathfrak{\jmath}(\mathfrak{q}) \cap \kappa_{0}$, and $\operatorname{Lie}\left(\mathbf{N}_{\mathfrak{q}}\right)=\mathfrak{L}_{0}(\mathfrak{q})=\mathfrak{L}(\mathfrak{q}) \cap \kappa_{0}$.

The obvious equivariant fibrations of complex flag manifolds yield the inclusions

$$
\mathbf{N}_{\mathfrak{q}_{1}} \subset \mathbf{N}_{\mathfrak{q}_{2}} \quad \text { if } \mathfrak{q}_{1}, \mathfrak{q}_{2} \in \mathbb{P}_{\kappa} \text { and } \quad \mathfrak{q}_{1} \subset \mathfrak{q}_{2} .
$$

The existence of the parabolic regularization proves the following

Proposition 7.1. For all complex subalgebras $\mathfrak{v}$ of $\kappa$ the set

$$
\mathbb{P}_{K}(\mathfrak{v})=\left\{\mathfrak{q} \in \mathbb{P}_{K} \mid \mathfrak{v} \subset \mathfrak{q}, \quad \mathfrak{n}_{\kappa}(\mathfrak{v}) \cap \mathfrak{L}(\mathfrak{q})=\{0\}\right\}
$$

is nonempty.

Definition 7.2. We say that a $\mathfrak{q} \in \mathbb{P}_{\kappa}(\mathfrak{w})$ is $\mathbf{M}_{0}$-admissible if $\operatorname{Ad}_{\kappa}\left(\mathbf{M}_{0}\right)(\mathfrak{q})=\mathfrak{q}$.

This is equivalent to the condition that $\mathbf{M}_{0} \subset \mathbf{N}_{\mathrm{q}}$. Set

$$
\mathbb{P}_{\kappa}\left(\mathfrak{v}, \mathbf{M}_{0}\right)=\left\{\mathfrak{q} \in \mathbb{P}_{\kappa}(\mathfrak{p}) \mid \operatorname{Ad}_{\kappa}\left(\mathbf{M}_{0}\right)(\mathfrak{q})=\mathfrak{q}\right\} .
$$

Remark 7.3. The condition $\mathbf{M}_{0} \subset \mathbf{N}_{\mathfrak{q}}$ is always satisfied if $\mathbf{M}_{0}$ is connected, so that $\mathbb{P}_{\kappa}\left(\mathfrak{v}, \mathbf{M}_{0}\right)=\mathbb{P}_{\kappa}(\mathfrak{y})$ in this special case.

For instance, in Example 5.7, the isotropy subgroup $\mathbf{M}_{0}$ at the base point consists of the diagonal matrices of the form

$$
\operatorname{diag}\left(\exp \left(i t_{1}\right), \exp \left(i t_{2}\right), \exp \left(i t_{3}\right), \exp \left(i t_{2}\right), \exp \left(i t_{1}\right)\right), \quad \text { with } 2 t_{1}+2 t_{2}+t_{3}=0 .
$$

and hence is connected. In fact, this is always the case when $M$ is the minimal orbit of a real form in a complex flag manifold (see [3]).

If $\mathbf{M}_{0}^{0}$ is the connected component of the identity in $\mathbf{M}_{0}$, then $\tilde{M}=\mathbf{K}_{0} / \mathbf{M}_{0}^{0}$ is a $\mathbf{K}_{0}$-homogeneous $C R$ manifold with the same $C R$ algebra $\left(\kappa_{0}, \mathfrak{v}\right)$ at all points in the fiber of $p_{0}$. Hence the $\mathbf{K}_{0}$-equivariant covering map $\tilde{\pi}: \tilde{M} \rightarrow M$ is a $C R$ submersion and a local $C R$-diffeomorphism with finite discrete fiber.

Lemma 7.4. If $\mathfrak{q} \in \mathbb{P}_{\kappa}\left(\mathfrak{v}, \mathbf{M}_{0}\right)$, then

$$
\left\{\begin{aligned}
\operatorname{Ad}_{\kappa}\left(\mathbf{M}_{0}\right)\left(\mathfrak{n}_{\kappa}(\mathfrak{q})\right) & =\mathfrak{n}_{\kappa}(\mathfrak{q}), & \operatorname{Ad}_{\kappa}\left(\mathbf{M}_{0}\right)(\mathfrak{L}(\mathfrak{q})) & =\mathfrak{L}(\mathfrak{q}), \\
\operatorname{Ad}_{\kappa}\left(\mathbf{M}_{0}\right)(\mathfrak{s}(\mathfrak{q})) & =\mathfrak{s}(\mathfrak{q}), & \operatorname{Ad}_{\kappa}\left(\mathbf{M}_{0}\right)(\mathfrak{z}(\mathfrak{q})) & =\mathfrak{z}(\mathfrak{q}) .
\end{aligned}\right.
$$

Proof. The first equality follows because $\mathfrak{n}_{\kappa}(\mathfrak{q})$ is a characteristic ideal; the second because $\mathbf{M}_{0} \subset \mathbf{K}_{0}$, and $\mathfrak{L}(\mathfrak{q})$ is the unique $\sigma$-invariant reductive complement of $\mathfrak{n}_{\kappa}(\mathfrak{q})$ in $q$. Here $\sigma$ is the conjugation of $\kappa$ with respect to the real form $\kappa_{0}$. Stabilizing $\mathfrak{L}(\mathfrak{q}), \operatorname{Ad}_{\mathfrak{\kappa}}\left(\mathbf{M}_{0}\right)$ also stabilizes its center and its semisimple ideal.

Proposition 7.5. If $\mathfrak{q} \in \mathbb{P}_{\kappa}\left(\mathfrak{v}, \mathbf{M}_{0}\right)$, then the inclusion $\mathbf{M}_{0} \subset \mathbf{N}_{\mathfrak{q}}$ defines a $\mathbf{K}_{0^{-}}$ equivariant smooth submersion

$$
\pi_{\mathfrak{q}}: M \rightarrow N_{\mathfrak{q}},
$$

which is a CR map. If $M$ is $\mathfrak{n}$-reductive, then $\pi_{\mathfrak{q}}$ has totally real fibers. 
Proof. We only need to prove the last statement. Assume that $M$ is n-reductive. Every $X \in \mathfrak{v}$ uniquely decomposes as a sum $X=Y+Z$, with $Y \in \mathfrak{n}_{\kappa}(\mathfrak{v}), Z \in \mathfrak{m}$. Since $\mathfrak{m} \subset \mathfrak{L}(\mathfrak{q}), X$ belongs to $\mathfrak{v} \cap \overline{\mathfrak{q}}$ if and only if $Y \in \mathfrak{v} \cap \mathfrak{L}(\mathfrak{q})$. But then $Y=0$, because $\mathfrak{n}_{\kappa}(\mathfrak{v}) \cap \mathfrak{L}(\mathfrak{q})=\{0\}$. This shows that $\mathfrak{v} \cap \overline{\mathfrak{q}}=\mathfrak{m}$. Therefore the fibers of $\pi_{\mathfrak{q}}$ are totally real by the criterion (3.7) of Proposition 3.10.

Proposition 7.6. (1) Let $\mathfrak{q}_{1}, \mathfrak{q}_{2} \in \mathbb{P}_{\kappa}(\mathfrak{v})$, with $\mathfrak{q}_{1} \subset \mathfrak{q}_{2}$. If $\mathfrak{q}_{1} \in \mathbb{P}_{\kappa}\left(\mathfrak{v}, \mathbf{M}_{0}\right)$, then also $\mathfrak{q}_{2} \in \mathbb{P}_{\kappa}\left(\mathfrak{v}, \mathbf{M}_{0}\right)$.

(2) The parabolic regularization $\mathfrak{w}$ of $\mathfrak{v}$ belongs to $\mathbb{P}_{\kappa}\left(\mathfrak{v}, \mathbf{M}_{0}\right)$.

(3) In particular, if $\mathfrak{q} \in \mathbb{P}_{\kappa}(\mathfrak{v})$ and $\mathfrak{w} \subset \mathfrak{q}$ then $\mathfrak{q} \in \mathbb{P}_{\kappa}\left(\mathfrak{v}, \mathbf{M}_{0}\right)$.

Proof. (1) follows from (7.2).

(2). Consider the sequence of Lie subalgebras defined by (6.1). For each $i=$ $1,2, \ldots$, set

$$
\mathbf{N}_{\mathfrak{v}_{i}}=\left\{g \in \mathbf{K}_{0} \mid \operatorname{Ad}(g)\left(\mathfrak{v}_{i}\right)=\mathfrak{v}_{i}\right\}, \quad N_{i}=\mathbf{K}_{0} / \mathbf{N}_{\mathfrak{v}_{i}} .
$$

Since $\mathfrak{v}$ is $\operatorname{Ad}\left(\mathbf{M}_{0}\right)$-invariant, also its characteristic ideal $\mathfrak{n}_{\kappa}(\mathfrak{v})$ and its normalizer $\mathfrak{v}_{1}$ are $\operatorname{Ad}\left(\mathbf{M}_{0}\right)$-invariant. Therefore $\mathbf{M}_{0} \subset \mathbf{N}_{\mathfrak{v}_{1}}$.

Let $i \geq 1$. If $g \in \mathbf{N}_{\mathfrak{v}_{i}}$ and $X \in \mathfrak{v}_{i+1}=\mathbf{N}_{\kappa}\left(\mathfrak{n}_{\kappa}\left(\mathfrak{v}_{i}\right)\right)$, we have

$$
\begin{array}{r}
{\left[\operatorname{Ad}_{\kappa}(g)(X), Y\right]=\operatorname{Ad}_{\kappa}(g)\left(\left[X, \operatorname{Ad}_{\kappa}\left(g^{-1}\right)(Y)\right]\right) \in \operatorname{Ad}_{\kappa}(g)\left(\mathfrak{n}_{\kappa}\left(\mathfrak{v}_{i}\right)\right)=\mathfrak{n}_{\kappa}\left(\mathfrak{p}_{i}\right),} \\
\forall Y \in \mathfrak{n}_{\kappa}\left(\mathfrak{v}_{i}\right),
\end{array}
$$

showing that $\operatorname{Ad}_{\kappa}(g)(X) \in \mathfrak{v}_{i+1}$. Therefore we have $\mathbf{N}_{\mathfrak{v}_{i}} \subset \mathbf{N}_{\mathfrak{v}_{i+1}}$. Hence, with $\mathfrak{w}=$ $\mathfrak{v}_{m}$, we obtain $\mathbf{M}_{0} \subset \mathbf{N}_{\mathfrak{v}_{1}} \subset \mathbf{N}_{\mathfrak{v}_{2}} \subset \cdots \subset \mathbf{N}_{\mathfrak{v}_{m}}=\mathbf{N}_{\mathfrak{w}}$, proving that $\mathfrak{w} \in \mathbb{P}_{\kappa}\left(\mathfrak{v}, \mathbf{M}_{0}\right)$.

Proposition 7.7. Let $\mathfrak{v}$ be a complex Lie subalgebra of $\kappa$. If $\mathfrak{q}$ is any maximal (with respect to inclusion) element of $\mathbb{P}_{\kappa}(\mathfrak{v})$, then

$$
\mathfrak{q}=\mathscr{L}\left(\mathfrak{n}_{\kappa}(\mathfrak{p})+\mathfrak{L}(\mathfrak{q})\right) \text {. }
$$

Proof. Let $\mathfrak{q}$ be any maximal element of $\mathbb{P}_{\kappa}(\mathfrak{y})$ and fix a maximal torus $t_{0}$ in $\mathscr{L}(\mathfrak{q})$. With $z=z(q)$, following [14], we define the $z$-roots as the non zero elements $v$ of the dual $3_{\mathbb{R}}^{*}$ of $\jmath_{\mathbb{R}}=i_{\jmath_{0}}(\mathfrak{q})$ for which

$$
\kappa_{v}=\left\{X \in \kappa \mid[Z, X]=v(Z) X, \forall Z \in \jmath_{\mathbb{R}}\right\} \neq\{0\} .
$$

The set $\mathcal{Z}$ of the 3 -roots is a finite subset of $\gamma_{\mathbb{R}}^{*}$, with the properties that

$\left\{\begin{array}{l}v \in \mathcal{Z} \Longrightarrow-v \in \mathcal{Z} ; \\ \kappa_{v_{1}+v_{2}}=\left[\kappa_{v_{1}}, \kappa_{v_{2}}\right] ; \\ \text { for all } v \in \mathcal{Z}, \kappa_{v} \text { is an irreducible } \mathfrak{L}(\mathfrak{q}) \text {-module; } \\ \text { there exists an ordering in } z_{\mathbb{R}}^{*} \text { such that } \mathfrak{n}_{\kappa}(\mathfrak{q})=\sum_{v>0} \kappa_{v} ; \\ \text { there is a system of linearly independent positive simple roots } v_{1}, \ldots, v_{\ell} \text { such } \\ \text { that every positive } v \in \mathcal{Z} \text { uniquely decomposes as } v=\sum_{i=1}^{\ell} c_{i} v_{i}, \text { with } c_{i} \in \mathbb{Z}_{+} .\end{array}\right.$

We claim that $\kappa_{v_{i}} \cap\left(\mathfrak{n}_{\kappa}(\mathfrak{p})+\mathfrak{I}(\mathfrak{q})\right) \neq\{0\}$ for $i=1, \ldots, \ell$. Assume by contradiction that there is $j$, with $1 \leq j \leq \ell$, such that $\kappa_{v_{j}} \cap\left(\mathfrak{n}_{\kappa}(\mathfrak{p})+\mathfrak{L}(\mathfrak{q})\right)=\{0\}$. Then we consider the parabolic $\mathfrak{q}^{\prime}=\mathfrak{q} \oplus \kappa_{-v_{j}}$. Since the elements $X$ of $\mathfrak{n}_{\kappa}(\mathfrak{p})$ uniquely decompose as sums $X=X_{0}+\sum_{v>0} X_{v}$, with $X_{0} \in \mathfrak{R}(\mathfrak{q})$ and $X_{v} \in \kappa_{v}$, we have

$$
\mathfrak{L}\left(\mathfrak{q}^{\prime}\right) \cap \mathfrak{n}_{\mathfrak{K}}(\mathfrak{v})=\left(\mathfrak{L}(\mathfrak{q}) \oplus \kappa_{v_{j}} \oplus \kappa_{-v_{j}}\right) \cap \mathfrak{n}_{\kappa}(\mathfrak{p})=\left(\mathfrak{L}(\mathfrak{q}) \oplus \kappa_{v_{j}}\right) \cap \mathfrak{n}_{\kappa}(\mathfrak{p})=\{0\} .
$$

Indeed, if $Y=X_{0}+X_{v_{j}}=Y$, with $Y \in \mathfrak{n}_{\kappa}(\mathfrak{v}), X_{0} \in \mathfrak{L}(\mathfrak{q}), X_{v_{j}} \in \kappa_{v_{j}}$, then $X_{v_{j}}=0$, because $X_{v_{j}} \in\left(\mathfrak{L}(\mathfrak{q})+\mathfrak{n}_{\kappa}(\mathfrak{p})\right) \cap \kappa_{v_{j}}=\{0\}$. Hence $Y=0$, because $X_{0}=Y \in$ $\mathfrak{L}(\mathfrak{q}) \cap \mathfrak{n}_{\kappa}(\mathfrak{v})=\{0\}$ 
This would give $\mathfrak{q} \varsubsetneqq \mathfrak{q}^{\prime} \in \mathbb{P}_{\kappa}(\mathfrak{v})$, contradicting the maximality of $\mathfrak{q}$.

Therefore

$$
\begin{aligned}
& \kappa_{v_{i}} \subset \mathfrak{n}_{\kappa}(\mathfrak{p})+\mathfrak{L}(\mathfrak{q})+\left[\mathfrak{Q}(\mathfrak{q}), \mathfrak{n}_{\kappa}(\mathfrak{p})\right]+\left[\mathfrak{Q}(\mathfrak{q}),\left[\mathfrak{L}(\mathfrak{q}), \mathfrak{n}_{\kappa}(\mathfrak{p})\right]\right]+ \cdots \\
& \subset \mathscr{L}\left(\mathfrak{n}_{\kappa}(\mathfrak{p})+\mathfrak{L}(\mathfrak{q})\right),
\end{aligned}
$$

and this implies (7.7).

We obtain, from Proposition 7.5 and Proposition 7.7

Theorem 7.8 (the deployment theorem). If $M$ is $\mathfrak{n}$-reductive, then for all maximal $\mathfrak{q}$ in $\mathbb{P}_{\mathrm{K}}\left(\mathfrak{v}, \mathbf{M}_{0}\right)$, (7.6) is a CR-deployment.

The connected compact Lie group $\mathbf{K}_{0}$ admits a linearization and hence a complexification $\mathbf{K}$, which is unique modulo isomorphisms. Let $V=\mathbf{K} / \mathbf{V}$ be the K-realization of $M$ (see Theorem 4.5) and $N_{\mathfrak{q}}=\mathbf{K} / \mathbf{Q}$ the complex flag manifold associated to the parabolic $\mathfrak{q}$ of $\kappa$. We have:

Theorem 7.9 (factoring through the realization). Let $\mathfrak{q} \in \mathbb{P}_{\kappa}\left(\mathfrak{v}, \mathbf{M}_{0}\right)$ and $\mathbf{Q}$ be the analytic Lie subgroup of $\mathbf{K}$ with $\operatorname{Lie}(\mathbf{Q})=\mathfrak{q}$. Then $\mathbf{V} \subset \mathbf{Q}$, and we obtain $a$ commutative diagram:

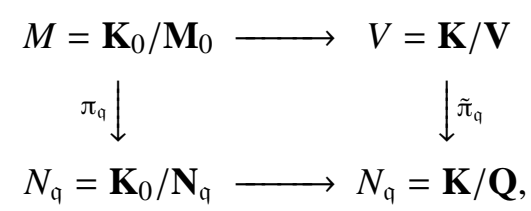

where the top horizontal arrow is a generic CR-embedding and a $\mathbf{K}$-realization, the right vertical arrow is a holomorphic submersion and the bottom horizontal arrow is an extension of the holomorphic action of $\mathbf{K}_{0}$ on $N_{\mathfrak{q}}$ to a holomorphic action of its complexification $\mathbf{K}$. Hence $\pi_{\mathfrak{q}}$ is the restriction to $M$ of a holomorphic submersion of the ambient space $V$.

If, moreover, $\mathfrak{n}_{\mathfrak{K}}(\mathfrak{v}) \subset \mathfrak{n}_{\mathfrak{K}}(\mathfrak{q})$, then the fibers of $\tilde{\pi}_{\mathfrak{q}}$ are Stein.

Proof. We know from Theorem 4.5 that the complex Lie subgroup $\mathbf{V}$ has a LeviChevalley decomposition $\mathbf{V}=\mathbf{V}_{n} \mathbf{M}$, where $\mathbf{V}_{n}$ is the analytic Lie subgroup of $\mathbf{K}$ with Lie algebra $\mathfrak{n}_{\kappa}(\mathfrak{v})$ and $\mathbf{M}=\left\{g \exp (i X) \mid g \in \mathbf{M}_{0}, X \in \mathfrak{m}_{0}\right\}$. If $\mathfrak{q} \in \mathbb{P}_{\kappa}\left(\mathfrak{v}, \mathbf{M}_{0}\right)$, then $\mathbf{M}_{0} \subset \mathbf{Q}$ and hence also $\mathbf{V} \subset \mathbf{Q}$ because $\mathbf{M}_{0}$ is a maximal compact subgroup of $\mathbf{V}$, and hence all connected components of $\mathbf{V}$ intersect $\mathbf{M}_{0}$. This gives the first part of the statement.

Assume now that $\mathfrak{q} \in \mathbb{P}_{\kappa}\left(\mathfrak{v}, \mathbf{M}_{0}\right)$ and $\mathfrak{n}_{\kappa}(\mathfrak{p}) \subset \mathfrak{n}_{\kappa}(\mathfrak{q})$. Then $\mathbf{Q}$ has a Levi-Chevalley decomposition $\mathbf{Q}=\mathbf{Q}_{n} \mathbf{Q}_{r}$, with analytic complex Lie subgroups $\mathbf{Q}_{n}, \mathbf{Q}_{r}$ with $\operatorname{Lie}\left(\mathbf{Q}_{n}\right)=\mathfrak{n}_{\mathfrak{K}}(\mathfrak{q}), \operatorname{Lie}\left(\mathbf{Q}_{r}\right)=\mathfrak{Q}(\mathfrak{q})$.

Since $\mathbf{M} \subset \mathbf{Q}_{r}$ and $\mathbf{Q}_{n}$ is a normal Lie subgroup of $\mathbf{Q}$, the product

$$
\mathbf{Q}_{n} \mathbf{M}=\left\{g_{1} g_{2} \mid g_{1} \in \mathbf{Q}_{n}, g_{2} \in \mathbf{M}\right\}
$$

is a closed Lie subgroup of $\mathbf{K}$. Since we assumed that $\mathfrak{n}_{\kappa}(\mathfrak{v}) \subset \mathfrak{n}_{\kappa}(\mathfrak{q})$, we have $\mathbf{V}_{n} \subset \mathbf{Q}_{n}$ and thus the inclusions $\mathbf{V} \subset \mathbf{Q}_{n} \mathbf{M} \subset \mathbf{Q}$ yield a commutative diagram

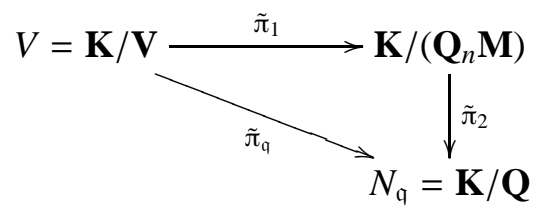


of $\mathbf{K}$-equivariant maps. The fiber of $\tilde{\pi}_{1}$ is $\left(\mathbf{Q}_{n} \mathbf{M}\right) / \mathbf{V} \simeq \mathbf{Q}_{n} /\left(\mathbf{Q}_{n} \cap \mathbf{V}\right)$, and therefore is complex Euclidean. The fiber of $\pi_{2}$ is $\mathbf{Q} /\left(\mathbf{Q}_{n} \mathbf{M}\right) \simeq \mathbf{Q}_{r} / \mathbf{M}$ and hence Stein because $\mathbf{Q}_{r}$ is Stein and $\mathbf{M}$ is the complexification of the compact subgroup $\mathbf{M}_{0}$ (see [16]). Then $\mathbf{Q} / \mathbf{V}$ is the total space of a fibration $\mathbf{Q} / \mathbf{V} \rightarrow \mathbf{Q} /\left(\mathbf{Q}_{n} \mathbf{M}\right)$ with a Stein base and complex Euclidean fibers and therefore is Stein by [17].

Example 7.10. In the proof of Proposition 7.7 we show that the root spaces corresponding to the simple positive 3 -roots belong to the $\mathfrak{L}(\mathfrak{q})$-module generated by $\mathfrak{n}_{\kappa}(\mathfrak{v})+\mathfrak{L}(\mathfrak{q})$. A natural question arises whether, in the definition of $C R$-spread, one could substitute to $\mathscr{L}\left(\mathfrak{n}_{\kappa}(\mathfrak{v})+\mathfrak{L}(\mathfrak{q})\right)$ the $\mathfrak{L}(\mathfrak{q})$-module generated by $\mathfrak{n}_{\kappa}(\mathfrak{v})+\mathfrak{L}(\mathfrak{q})$. We provide an example showing that the statement of Theorem 7.8 does not hold with this stronger notion of $C R$-deployment.

Let $\kappa_{0}=\mathfrak{s} \mathfrak{D}(7)$. Fix a maximal torus $t_{0}$ in $\kappa_{0}$, let $\mathcal{K}=\left\{ \pm e_{i}, \pm e_{j} \pm e_{h} \mid 1 \leq\right.$ $i \leq 3,1 \leq j<h \leq 3\}$ the root space of $(\kappa, \mathrm{t})$. Take $\mathfrak{v}=\kappa^{e_{1}-e_{3}} \oplus \kappa^{e_{2}}$. This is an Abelian Lie algebra of nilpotent elements. The parabolic regularization $\mathfrak{w}$ of $\mathfrak{v}$ is the Borel subalgebra associated to the standard ordering of $\mathcal{K}$, corresponding to the cross-marked Satake diagram:

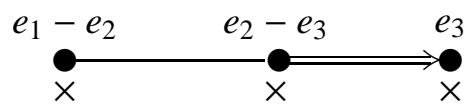

There are exactly two maximal parabolic subalgebra in $\mathbb{P}_{\kappa}(\mathfrak{v})$ which contain $\mathfrak{w}$.

The first maximal $\mathfrak{q} \in \mathbb{P}_{\kappa}(\mathfrak{v})$, with $\mathfrak{w} \subset \mathfrak{q}$, is associated to the cross-marked Satake diagram

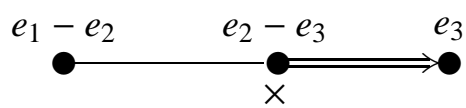

We have $Q_{n}=\left\{e_{1}, e_{2}, e_{1} \pm e_{3}, e_{2} \pm e_{3}, e_{1}+e_{2}\right\}, Q_{r}=\left\{ \pm e_{3}, \pm\left(e_{1}-e_{2}\right)\right\}$ and, accordingly, the centralizer $z$ of $\mathfrak{L}(\mathfrak{q})$ in $\mathrm{t}$ consists of the elements $H \in \mathrm{t}$ with $e_{1}(H)=e_{2}(H)$ and $e_{3}(H)=0$. The $z$-roots are $\{ \pm v, \pm 2 v\}$, with

$$
\begin{aligned}
\kappa_{v} & =\kappa^{e_{1}} \oplus \kappa^{e_{2}} \oplus \kappa^{e_{1}-e_{3}} \oplus \kappa^{e_{1}+e_{3}} \oplus \kappa^{e_{2}-e_{3}} \oplus \kappa^{e_{2}+e_{3}}, \\
\kappa_{2 v} & =\kappa^{e_{1}+e_{2}} .
\end{aligned}
$$

We note that $\mathfrak{p} \subset \kappa_{v}$. Hence $\mathfrak{q}$ is generated by $\mathfrak{v}+\mathfrak{Q}(\mathfrak{q})$ as a Lie algebra, but not as an $\mathfrak{L}(\mathfrak{q})$-Lie module.

The other possible choice of a maximal $\mathfrak{q} \in \mathbb{P}_{\kappa}(\mathfrak{y})$ with $\mathfrak{w} \subset \mathfrak{q}$ corresponds to the cross-marked Satake diagram

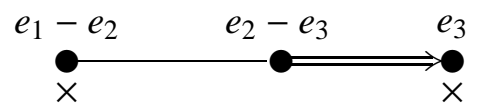

In this case, $Q_{n}=\left\{e_{1}, e_{2}, e_{3}, e_{1} \pm e_{2}, e_{1} \pm e_{3}, e_{2}+e_{3}\right\}, Q_{r}=\left\{ \pm\left(e_{2}-e_{3}\right)\right\}$. The corresponding set of $z$-roots yields

$$
\begin{aligned}
\kappa_{v_{1}} & =\left\{e_{1}-e_{2}, e_{1}-e_{3}\right\}, \\
\kappa_{v_{2}} & =\left\{e_{2}, e_{3}\right\}, \\
\kappa_{v_{1}+v_{2}} & =\left\{e_{1}\right\}, \\
\kappa_{v_{1}+2 v_{2}} & =\left\{e_{1}+e_{2}, e_{1}+e_{3}\right\}, \\
\kappa_{2 v_{2}} & =\left\{e_{2}+e_{3}\right\} .
\end{aligned}
$$


We note that $\mathfrak{v} \cap \kappa_{v_{1}}=\kappa^{e_{1}-e_{2}} \neq\{0\}, \mathfrak{v} \cap \kappa_{v_{2}}=\kappa^{e_{3}} \neq\{0\}$, and $\mathfrak{v}=\mathfrak{v} \cap \kappa_{v_{1}} \oplus \mathfrak{v} \cap \kappa_{v_{2}}$. Also in this case $\mathfrak{v}+\mathfrak{L}(\mathfrak{q})$ generates $\mathfrak{q}$ as a Lie subalgebra, but not as an $\mathfrak{L}(\mathfrak{q})$-Lie module.

This example also shows that, in general, deployments corresponding to different maximal elements of $\mathbb{P}_{\kappa}\left(\mathfrak{v}, \mathbf{M}_{0}\right)$ may be non equivalent.

7.1. Characterization of $\mathbb{P}_{\kappa}\left(\mathfrak{v}, \mathbf{M}_{0}\right)$. For every $A \in \kappa_{0}, a_{\kappa}(A)$ is semisimple and has purely imaginary eigenvalues. We associate to $A \in \kappa_{0}$ the parabolic subalgebra

$$
\mathfrak{q}_{A}=\sum_{\lambda \geq 0}\{X \in \kappa \mid[A, X]=i \lambda X\} .
$$

As we already noted in $\$ 5.1$, the corresponding complex manifold $N_{\mathrm{q}_{A}}$ is the totally complex $\mathbf{K}_{0}$-homogeneous $C R$ manifold with isotropy

$$
\mathbf{N}_{\mathrm{q}_{A}}=\left\{g \in \mathbf{K}_{0} \mid \operatorname{Ad}_{\kappa}(g)(A)=A\right\}
$$

and $C R$ algebra $\left(\kappa_{0}, \mathfrak{q}_{A}\right)$ at the base point $p_{0}$.

Theorem 7.11. With

$$
\mathfrak{a}_{0}\left(\mathbf{M}_{0}\right)=\left\{A \in \kappa_{0} \mid \operatorname{Ad}_{\kappa}(g)(A)=A, \forall g \in \mathbf{M}_{0}\right\}
$$

we have:

$$
\mathbb{P}_{\kappa}\left(\mathfrak{v}, \mathbf{M}_{0}\right)=\left\{\mathfrak{q}_{A} \mid A \in \mathfrak{a}_{0}\left(\mathbf{M}_{0}\right), \quad \mathfrak{v} \subset \mathfrak{q}_{A}\right\}
$$

Proof. Let us denote by $\mathcal{P}$ the right hand side of (7.11). By (7.9), we have the inclusion $\mathbb{P}_{\kappa}\left(\mathfrak{v}, \mathbf{M}_{0}\right) \subset \mathcal{P}$. The opposite inclusion is obvious, and hence we have equality.

Example 7.12. Let $\mathbf{K}_{0}$ be a connected compact Lie group, $t_{0}$ a maximal torus in $\mathbf{K}_{0}$ and $\mathbf{M}_{0}$ the normalizer of $t_{0}$ in $\mathbf{K}_{0}$. If $\mathbf{T}_{0}$ is the analytic subgroup with $\operatorname{Lie}\left(\mathbf{T}_{0}\right)=t_{0}$, then $\mathbf{T}_{0}$ is a normal subgroup of $\mathbf{M}_{0}$ and the quotient $\mathbf{M}_{0} / \mathbf{T}_{0}$ is the Weyl group. Consider on $M=\mathbf{K}_{0} / \mathbf{M}_{0}$ the totally real $C R$ structure defined at the base point by the $C R$ algebra $\left(\kappa_{0}, \mathrm{t}\right)$, with $\mathrm{t}=\mathbb{C} \otimes_{\mathbb{R}} \mathrm{t}_{0}$. Then $\mathbb{P}_{\kappa}\left(\mathrm{t}, \mathbf{M}_{0}\right)$ only contains $\kappa$. Thus there are no $C R$ structures of type $M_{\mathfrak{q}}$ with positive $C R$ dimension. This shows that, in general, the set $\left\{A \in \kappa_{0} \mid\left[A, \mathfrak{m}_{0}\right]=\{0\}, \mathfrak{v} \subset \mathfrak{q}_{A}\right\}$, when $\mathbf{M}_{0}$ is not connected, can be larger than $\mathfrak{a}_{0}\left(\mathbf{M}_{0}\right)$.

7.2. The deployment theorem for homogeneous foliated complex manifolds. With a proof similar to that of Theorem 7.9 we obtain

Theorem 7.13. Let $\mathbf{V}$ be a decomposable subgroup of a complex reductive Lie group $\mathbf{K}$, and $V=\mathbf{K} / \mathbf{V}$. Fix a Levi-Chevalley decomposition (4.3) of $\mathbf{V}$ and let $\left(M^{\mathbb{C}}, \mathscr{D}_{\mathfrak{v}}\right)$, with $\mathfrak{v}=\operatorname{Lie}(\mathbf{V})$, be the corresponding Stein lift of $V$ of $\$ 4.7 \$ 4.8$

Let $\mathfrak{q}$ be maximal in $\mathbb{P}_{\kappa}(\mathfrak{y})$ and $\mathbf{Q}$ the corresponding parabolic subgroup of $\mathbf{K}$. Then $\mathbf{V} \subset \mathbf{Q}$ and $\mathbf{Q}$ has a Levi-Chevalley decomposition $\mathbf{Q}=\mathbf{Q}_{n} \cdot \mathbf{Q}_{r}$ with $\mathbf{M} \subset \mathbf{Q}_{r}$, $\mathbf{V}_{n} \cap \mathbf{Q}_{r}=\{1\}$.

Let $N_{\mathfrak{q}}=\mathbf{K} / \mathbf{Q}$ and $\left(N^{\mathbb{C}}, \mathscr{D}_{\mathfrak{q}}\right)$, with $N_{\mathfrak{q}}^{\mathbb{C}}=\mathbf{K} / \mathbf{Q}_{r}$, its Stein lift. Then we have a commutative diagram

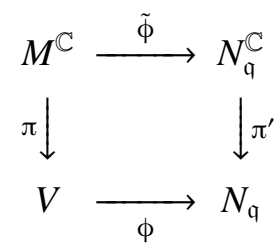


of $\mathbf{K}$-equivariant fibrations, and moreover $\phi, \phi_{r}$ have Stein fibers, and $\tilde{\phi}$ is a deployment of foliated complex manifolds.

\section{LIFTED $C R$ STRUCTURES}

We keep the notation of $\$ 7$ Our next goal is to describe all horocyclic $\mathfrak{n -}$ reductive $\mathbf{K}_{0}$-homogeneous $C R$ structures of a given $\mathbf{K}_{0}$-space $M$. In fact, we take up the slightly more general question of strengthening a given $\mathrm{n}$-reductive $\mathbf{K}_{0}$-homogeneous $C R$ structure on $M$. The totally real $M$ corresponds indeed to the choice of the trivial n-reductive $\mathbf{K}_{0}$-equivariant $C R$ structure defined at $p_{0}$ by $\left(\kappa_{0}, \mathfrak{m}\right)$. Throughout this section we will always assume that $M$ is n-reductive, i.e. that

$$
\mathfrak{v}=\mathfrak{m} \oplus \mathfrak{n}_{\kappa}(\mathfrak{v}), \quad \text { with } \quad \mathfrak{m}=\mathfrak{L}(\mathfrak{v})=\mathfrak{v} \cap \overline{\mathfrak{v}} .
$$

We begin with a simple algebraic lemma.

Lemma 8.1. If $\mathfrak{q} \in \mathbb{P}_{\kappa}(\mathfrak{v})$, then $\mathfrak{v}_{\mathfrak{q}}=\mathfrak{v}+\mathfrak{n}_{\kappa}(\mathfrak{q})$ is a complex Lie algebra with $\mathfrak{n}_{\kappa}\left(\mathfrak{v}_{\mathfrak{q}}\right)=$ $\mathfrak{n}_{\mathrm{K}}(\mathfrak{v})+n_{\mathrm{K}}(\mathfrak{q})$, and admits a Levi-Chevalley decomposition with reductive Levi factor m. In particular, $\mathfrak{Q}\left(\mathfrak{v}_{\mathfrak{q}}\right)=\mathfrak{v}_{\mathfrak{q}} \cap \overline{\mathfrak{v}}_{\mathfrak{q}}=\mathfrak{m}$.

Proof. If $\mathfrak{q} \in \mathbb{P}_{\kappa}(\mathfrak{v})$, then $\mathfrak{v} \subset \mathfrak{q}$ and hence $\left[\mathfrak{v}, \mathfrak{n}_{\kappa}(\mathfrak{q})\right] \subset\left[\mathfrak{q}, \mathfrak{n}_{\kappa}(\mathfrak{q})\right] \subset \mathfrak{n}_{\kappa}(\mathfrak{q})$ shows that $\mathfrak{v}_{\mathfrak{q}}=\mathfrak{v}+\mathfrak{n}_{\kappa}(\mathfrak{q})$ is a Lie subalgebra of $\kappa$, containing $\mathfrak{n}_{\kappa}(\mathfrak{q})$ as an ideal of nilpotent elements. Moreover, $\mathfrak{n}_{\kappa}(\mathfrak{v})+\mathfrak{n}_{\kappa}(\mathfrak{q})$ is also an ideal in $\mathfrak{v}_{\mathfrak{q}}$ which consists of nilpotent elements and has the reductive complement $\mathrm{m}$.

Proposition 8.2. Let $\mathfrak{q} \in \mathbb{P}_{\kappa}\left(\mathfrak{v}, \mathbf{M}_{0}\right)$, and set $\mathfrak{v}_{\mathfrak{q}}=\mathfrak{v}+\mathfrak{n}_{\kappa}(\mathfrak{q})$. Then the $C R$ algebra $\left(\kappa_{0}, \mathfrak{v}_{\mathfrak{q}}\right)$ at the base point defines on $M=\mathbf{K}_{0} / \mathbf{M}_{0}$ another $\mathfrak{n}$-reductive $\mathbf{K}_{0}$ equivariant CR structure, which strengthens the one defined by $\left(\kappa_{0}, \mathfrak{v}\right)$.

Proof. It suffices to notice that, since $\mathfrak{q} \in \mathbb{P}_{\kappa}\left(\mathfrak{v}, \mathbf{M}_{0}\right)$, the subalgebras $\mathfrak{v}$ and $\mathfrak{n}_{\kappa}(\mathfrak{q})$ are both $\operatorname{Ad}_{\kappa}\left(\mathbf{M}_{0}\right)$-invariant. Hence $\mathfrak{v}_{\mathfrak{q}}$ is $\operatorname{Ad}_{\kappa}\left(\mathbf{M}_{0}\right)$-invariant. Using Lemma8.1, the statement follows from Proposition 3.3 .

Remark 8.3. The set $\mathbb{P}_{\kappa}\left(\mathfrak{m}, \mathbf{M}_{0}\right)$ parameterizes the horocyclic n-reductive $\mathbf{K}_{0^{-}}$ homogeneous $C R$ structures on $M=\mathbf{K}_{0} / \mathbf{M}_{0}$. These are the ones which are defined at the base point by a $C R$ algebra of the form $\left(\kappa_{0}, \mathfrak{m} \oplus \mathfrak{n}_{\kappa}(\mathfrak{q})\right)$, with $\mathfrak{q} \in \mathbb{P}_{\kappa}(\mathfrak{m})$.

Notation 8.4. We denote by $M_{\mathfrak{q}}$ the $\mathbf{K}_{0}$-homogeneous $C R$ manifold with isotropy $\mathbf{M}_{0}$ and $C R$ algebra $\left(\kappa_{0}, \mathfrak{v}_{\mathfrak{q}}\right)$ at the base point, described in Proposition 8.2.

We get from Proposition 7.5, Lemma 8.1 and Proposition 8.2

Theorem 8.5 (The lift theorem). For $\mathfrak{q} \in \mathbb{P}_{\kappa}\left(\mathfrak{v}, \mathbf{M}_{0}\right)$, the $\mathbf{K}_{0}$-equivariant map

$$
\phi_{\mathfrak{q}}: M_{\mathfrak{q}} \rightarrow N_{\mathfrak{q}}
$$

is a CR lift.

Proposition 8.6 (realization). Let $\mathbf{K}$ be a complexification of $\mathbf{K}_{0}$.

(1) For every $\mathfrak{q} \in \mathbb{P}_{\mathfrak{K}}\left(\mathfrak{v}, \mathbf{M}_{0}\right), M_{\mathfrak{q}}$ is $\mathbf{K}$-realizable.

(2) Let $\mathbf{V}_{\mathfrak{q}}$ be a complex Lie subgroup of $\mathbf{K}$ with $\operatorname{Lie}\left(\mathbf{V}_{\mathfrak{q}}\right)=\mathfrak{v}_{\mathfrak{q}}, \mathbf{V}_{\mathfrak{q}} \cap \mathbf{K}_{0}=\mathbf{M}_{0}$, and $\mathbf{Q}=\left\{g \in \mathbf{K} \mid \operatorname{Ad}_{\kappa}(g)(\mathfrak{q})=\mathfrak{q}\right\}$ the parabolic subgroup with $\operatorname{Lie}(\mathbf{Q})=\mathfrak{q}$. 
Then $\mathbf{V}_{\mathfrak{q}} \subset \mathbf{Q}$ and we obtain a commutative diagram

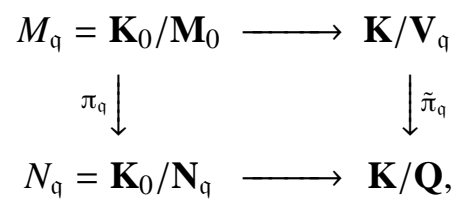

where the top horizontal arrow is the $\mathbf{K}$-realization of $M_{\mathfrak{q}}$, the bottom horizontal arrow is an extension of holomorphic action from $\mathbf{K}_{0}$ to $\mathbf{K}$, and the right vertical arrow $\tilde{\pi}_{\mathfrak{q}}$ is a $\mathbf{K}$-equivariant holomorphic fibration with Stein fibers.

Proof. It suffices to apply Theorem7.9 to $M_{\mathfrak{q}}$, using (2) of Proposition 8.13

8.1. Factorization of the lift. Let $\tau_{0}$ be a maximal torus of $m_{0}$. An element $q$ of $\mathbb{P}_{\kappa}\left(\mathfrak{v}, \mathbf{M}_{0}\right)$ contains a maximal torus $t_{0}$ of $\kappa_{0}$ with $t_{0} \cap m_{0}=\tau_{0}$. Set

$$
3_{0}=\left\{A \in \mathrm{t}_{0} \mid \operatorname{Ad}_{\kappa}(g)(A)=A, \forall g \in \mathbf{M}_{0}\right\}, \mathrm{Z}_{\mathrm{t}_{0}}\left(\mathrm{~m}_{0}\right)=\left\{A \in \mathrm{t}_{0} \mid\left[A, \mathrm{~m}_{0}\right]=\{0\}\right\} .
$$

We have $3_{0} \subset \mathrm{Z}_{\mathrm{t}_{0}}\left(\mathrm{~m}_{0}\right)$, with $z_{0}=\mathrm{Z}_{\mathrm{t}_{0}}\left(\mathrm{~m}_{0}\right)$ when $\mathbf{M}_{0}$ is connected, and $\mathfrak{q}=\mathfrak{q}_{A}$ for some $A \in 3_{0}$. The meaning of $Z_{\mathrm{t}_{0}}\left(\mathrm{~m}_{0}\right)$ is illustrated by the following

Lemma 8.7. The necessary and sufficient condition for $\mathrm{m}_{0}$ to be $\mathrm{t}_{0}$-regular is that

$$
\mathrm{t}_{0}=\tau_{0}+\mathrm{Z}_{\mathrm{t}_{0}}\left(\mathrm{~m}_{0}\right) .
$$

Proof. The condition is clearly sufficient. Vice versa, when $\mathrm{m}_{0}$ is $\mathrm{t}_{0}$-regular, $z_{0}$ is the center of the reductive subalgebra $t_{0}+m_{0}$, and $\tau_{0}$ contains the maximal torus $t_{0} \cap\left[\mathfrak{m}_{0}, \mathfrak{m}_{0}\right]$ of its semisimple ideal $\left[\mathfrak{m}_{0}, \mathfrak{m}_{0}\right]$, so that $(8.4)$ holds true.

Fix $\mathfrak{q} \in \mathbb{P}_{\kappa}\left(\mathfrak{v}, \mathbf{M}_{0}\right)$ and set

$$
\mathfrak{s}_{\mathfrak{q}}=\left[\mathfrak{L}_{0}(\mathfrak{q}), \mathfrak{L}_{0}(\mathfrak{q})\right], \quad \mathbf{S}_{\mathfrak{q}}=\text { analytic Lie subgroup with } \operatorname{Lie}\left(\mathbf{S}_{\mathfrak{q}}\right)=\mathfrak{s}_{\mathfrak{q}} .
$$

We note that the semisimple real Lie group $\mathbf{S}_{q}$ is compact and algebraic.

Lemma 8.8. We have $\operatorname{Ad}_{\kappa_{0}}(g)\left(\mathfrak{s}_{\mathfrak{q}}\right)=\mathfrak{s}_{\mathfrak{q}}$ and $\operatorname{ad}(g)\left(\mathbf{S}_{\mathfrak{q}}\right)=\mathbf{S}_{\mathfrak{q}}$ for all $g \in \mathbf{M}_{0}$.

Proof. From $\mathbf{M}_{0} \subset \mathbf{N}_{\mathfrak{q}}$ we have $\operatorname{Ad}_{\kappa_{0}}(g)\left(\mathfrak{L}_{0}(\mathfrak{q})\right)=\mathfrak{L}_{0}(\mathfrak{q})$, because $\operatorname{Lie}\left(\mathbf{N}_{\mathfrak{q}}\right)=\mathfrak{L}_{0}(\mathfrak{q})$. Then also the semisimple ideal $\mathfrak{s}_{\mathfrak{q}}$ of $\mathfrak{L}_{0}(\mathfrak{q})$ is $\operatorname{Ad}_{\mathfrak{\kappa}_{0}}\left(\mathbf{M}_{0}\right)$-invariant. Hence the analytic Lie subgroup $\mathbf{S}_{\mathfrak{q}}$ generated by $\mathfrak{s}_{\mathfrak{q}}$ is $\operatorname{ad}\left(\mathbf{M}_{0}\right)$-invariant.

Corollary 8.9. The product

$$
\mathbf{L}_{\mathfrak{q}}=\mathbf{M}_{0} \mathbf{S}_{\mathfrak{q}}=\left\{g_{1} g_{2} \mid g_{1} \in \mathbf{M}_{0}, g_{2} \in \mathbf{S}_{\mathfrak{q}}\right\}
$$

is a compact subgroup of $\mathbf{K}_{0}$, contained in $\mathbf{N}_{\mathrm{q}_{A}}$.

Proof. The product (8.6) is a group by Lemma 8.8 and is contained in $\mathbf{N}_{\mathrm{q}_{A}}$ because both $\mathbf{M}_{0}$ and $\mathbf{S}_{\mathrm{q}}$ are contained in $\mathbf{N}_{\mathrm{q}_{A}}$.

Lemma 8.10. The quotient $\mathbf{L}_{\mathfrak{q}} / \mathbf{M}_{0}$ is a homogeneous space of homotopic characteristic

$$
c_{\mathfrak{q}}=\operatorname{rank} \mathfrak{s}_{\mathfrak{q}}-\operatorname{dim}\left(\tau_{0} \cap \mathfrak{s}_{\mathfrak{q}}\right) .
$$

In particular, $\chi\left(\mathbf{L}_{\mathfrak{q}} / \mathbf{M}_{0}\right)>0$ if and only if $\mathrm{t}_{0} \cap \mathfrak{s}_{0}^{A} \subset \tau_{0}$. 
Proof. Indeed, $\tau_{0}+\left(t_{0} \cap \mathfrak{s}_{0}^{A}\right)$ is a maximal torus in $\mathfrak{I}_{\mathfrak{q}}=\operatorname{Lie}\left(\mathbf{L}_{\mathfrak{q}}\right)$. The homotopic characteristic of $\mathbf{L}_{\mathfrak{q}} / \mathbf{M}_{0}$ is the difference between the dimensions of a maximal torus of $\mathfrak{l}_{\mathfrak{q}}$ and of a maximal torus of $\mathfrak{m}_{0}$. This gives (8.7). The last claim follows because the Euler-Poincaré characteristic of $\mathbf{L}_{\mathfrak{q}} / \mathbf{M}_{0}$ is positive if and only if its homotopic characteristic is 0 .

Let $L_{\mathrm{q}}$ be the $\mathbf{K}_{0}$-homogeneous $C R$ manifold with isotropy $\mathbf{L}_{\mathrm{q}}$ and $C R$ algebra $\left(\kappa_{0}, \mathfrak{m}+\mathfrak{n}_{\kappa}(\mathfrak{q})+\mathbb{C} \otimes_{\mathbb{R}} \mathfrak{s}_{\mathfrak{q}}\right)$ at the base point.

By the preceding discussion we obtain:

Proposition 8.11. Let $\mathfrak{q} \in \mathbb{P}_{\kappa}\left(\mathfrak{v}, \mathbf{M}_{0}\right)$. Then (8.2) factors through $\mathbf{K}_{0}$-equivariant CR-lifts:

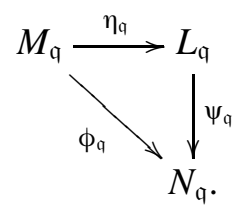

in which the fibers of $\eta_{\mathfrak{q}}$ are homogeneous spaces of homotopic characteristic $c_{\mathfrak{q}}$ and the fibers of $\psi_{\mathrm{q}}$ are real tori.

Proof. In fact the typical fiber of $\eta_{\mathfrak{q}}$ is $\mathbf{L}_{\mathfrak{q}} / \mathbf{M}_{0}$, and the fibers of $\psi_{\mathfrak{q}}$ are quotients of tori.

8.2. Maximal $C R$ structures. Proposition 7.6 shows that the problem of finding the $C R$-strengthenings $M_{\mathfrak{q}}$ of an $\mathfrak{n}$-reductive $M$ is equivalent to find the minimal elements of $\mathbb{P}_{\kappa}\left(\mathfrak{v}, \mathbf{M}_{0}\right)$. We shall prove that there is essentially a unique $\mathfrak{n}$-reductive maximal $C R$ structure on $M$ (see Remark 8.14).

Lemma 8.12. If $\mathfrak{q}_{1}, \mathfrak{q}_{2} \in \mathbb{P}_{\kappa}\left(\mathfrak{v}, \mathbf{M}_{0}\right)$, then

$$
\mathfrak{q}=\mathfrak{n}_{\kappa}\left(\mathfrak{q}_{1}\right)+\mathfrak{q}_{1} \cap \mathfrak{q}_{2}
$$

is a parabolic subalgebra of $\kappa$ which belongs to $\mathbb{P}_{\kappa}\left(\mathfrak{v}, \mathbf{M}_{0}\right)$.

Proof. Fix a Cartan subalgebra $t$ of $\kappa$ contained in $\mathfrak{q}_{1} \cap \mathfrak{q}_{2}$ and let $\mathcal{K}$ be the root system of $(\kappa, \mathrm{t})$. Then there are $A_{1}, A_{2} \in \mathrm{t}_{\mathbb{R}}=\{H \in \mathrm{t} \mid \operatorname{ad}(H)$ has real eigenvalues $\}$ such that

$$
\mathfrak{q}_{i}=\mathrm{t} \oplus \sum_{\alpha\left(A_{i}\right) \geq 0} \kappa^{\alpha}, \quad \text { for } i=1,2 .
$$

If $\epsilon>0$ is so small that $\left|\alpha\left(A_{2}\right)\right|<\epsilon^{-1}\left|\alpha\left(A_{1}\right)\right|$ for all roots $\alpha$ with $\alpha\left(A_{1}\right) \neq 0$, then

$$
\mathfrak{q}=\mathrm{t} \oplus \sum_{\alpha\left(A_{1}+\epsilon A_{2}\right) \geq 0} \kappa^{\alpha} .
$$

Indeed $\mathfrak{q}$ contains $\mathrm{t}$ and $\kappa^{\alpha} \subset \mathfrak{q}$ if and only if either $\alpha\left(A_{1}\right)>0$, or $\alpha\left(A_{1}\right)=0$ and $\alpha\left(A_{2}\right) \geq 0$. This proves that $\mathfrak{q}$ is parabolic in $\kappa$. It belongs to $\mathbb{P}_{\kappa}\left(\mathfrak{v}, \mathbf{M}_{0}\right)$ because, by Lemma 7.4, the right hand side of (8.8) is a sum of $\operatorname{Ad}_{\kappa}\left(\mathbf{M}_{0}\right)$-invariant subalgebras, and $\mathfrak{v} \subset \mathfrak{q}_{1} \cap \mathfrak{q}_{2}, \quad \mathfrak{n}_{\kappa}(\mathfrak{v}) \cap \mathfrak{Q}(\mathfrak{q}) \subset \mathfrak{n}_{\kappa}(\mathfrak{v}) \cap \mathfrak{L}\left(\mathfrak{q}_{1}\right)=\{0\}$.

Proposition 8.13. (1) If $\mathfrak{q}_{1}, \mathfrak{q}_{2}$ are minimal in $\mathbb{P}_{\mathfrak{K}}\left(\mathfrak{v}, \mathbf{M}_{0}\right)$, then $\mathfrak{Q}\left(\mathfrak{q}_{1}\right)$ and $\mathfrak{L}\left(\mathfrak{q}_{2}\right)$ are $\operatorname{Ad}_{\kappa}\left(\mathbf{K}_{0}\right)$-conjugate.

(2) For all minimal elements $\mathfrak{q}$ of $\mathbb{P}_{\kappa}\left(\mathfrak{v}, \mathbf{M}_{0}\right)$ we have $\mathfrak{n}_{\kappa}(\mathfrak{v}) \subset \mathfrak{n}_{\kappa}(\mathfrak{q})$.

Proof. Let $\mathfrak{q}_{1}, \mathfrak{q}_{2}$ be two elements of $\mathbb{P}_{\kappa}\left(\mathfrak{v}, \mathbf{M}_{0}\right)$. By Lemma8.12, $\mathfrak{q}=\mathfrak{n}_{\kappa}\left(\mathfrak{q}_{1}\right)+$ $\mathfrak{q}_{1} \cap \mathfrak{q}_{2}$ is parabolic and belongs to $\mathbb{P}_{\kappa}\left(\mathfrak{v}, \mathbf{M}_{0}\right)$. If $\mathfrak{q}_{1}$ is minimal, then $\mathfrak{q}=\mathfrak{q}_{1}$. Hence $\mathfrak{q}_{1} \cap \mathfrak{q}_{2}$ contains a reductive Levi-factor $v$ of $\mathfrak{q}_{1}$, which is also a maximal reductive subalgebra of $\mathfrak{q}_{1} \cap \mathfrak{q}_{2}$. If also $\mathfrak{q}_{2}$ is minimal, then, by the same argument, $v$ is also 
a reductive Levi factor of $\mathfrak{q}_{2}$. Thus we have $\mathfrak{L}\left(\mathfrak{q}_{1}\right) \sim v \sim \mathfrak{L}\left(\mathfrak{q}_{2}\right)$ with respect to $\operatorname{Int}(\kappa)$. Since both $\mathscr{L}\left(\mathfrak{q}_{1}\right)$ and $\mathfrak{L}\left(\mathfrak{q}_{2}\right)$ are complexifications of subalgebras of $\kappa_{0}$, they are also $\operatorname{Int}\left(\kappa_{0}\right)$-conjugate, and hence $\operatorname{Ad}_{\kappa}\left(\mathbf{K}_{0}\right)$-conjugate. This proves (1).

Let $\mathfrak{q}_{1}$ be any element of $\mathbb{P}_{\kappa}\left(\mathfrak{v}, \mathbf{M}_{0}\right)$ and $\mathfrak{q}_{2}$ be the parabolic regularization of $\mathfrak{v}$. We recall that $\mathfrak{q}_{2} \in \mathbb{P}_{\kappa}\left(\mathfrak{v}, \mathbf{M}_{0}\right)$ and $\mathfrak{n}_{\kappa}(\mathfrak{p}) \subset \mathfrak{q}_{2}$. Then $\mathfrak{q}=\mathfrak{n}_{\kappa}\left(\mathfrak{q}_{1}\right)+\mathfrak{q}_{1} \cap \mathfrak{q}_{2}$ belongs to $\mathbb{P}_{\kappa}\left(\mathfrak{v}, \mathbf{M}_{0}\right)$ and $\left(\mathfrak{n}_{\kappa}\left(\mathfrak{q}_{1}\right)+\mathfrak{n}_{\kappa}\left(\mathfrak{q}_{2}\right) \cap \mathfrak{q}_{1}\right) \subset \mathfrak{n}_{\kappa}(\mathfrak{q})$ because it is an ideal of $\mathfrak{q}$ consisting of nilpotent elements. If $\mathfrak{q}_{1}$ is minimal, we have $\mathfrak{q}=\mathfrak{q}_{1}$ and hence $\mathfrak{n}_{\kappa}(\mathfrak{q})=\mathfrak{n}_{\kappa}\left(\mathfrak{q}_{1}\right)$ yields $\mathfrak{n}_{\kappa}(\mathfrak{v}) \subset \mathfrak{n}_{\kappa}\left(\mathfrak{q}_{2}\right) \cap \mathfrak{q}_{1} \subset \mathfrak{n}_{\kappa}\left(\mathfrak{q}_{1}\right)$, proving (2).

Remark 8.14. If $\mathfrak{q}_{1}, \mathfrak{q}_{2} \in \mathbb{P}_{\kappa}$ have Int $(\kappa)$-conjugate reductive Levi factors, then there is an element $g \in \mathbf{K}_{0}$ such that $\mathfrak{g}_{2}=\operatorname{Ad}_{\kappa}(g)\left(\mathfrak{g}_{1}\right)$.

This follows from the observations that:

- $\operatorname{Ad}_{\kappa}\left(\mathbf{K}_{0}\right)$ is transitive on the $\operatorname{Int}(\kappa)$-orbit of $\mathfrak{q}_{1}$;

- hence, if $\mathfrak{L}\left(\mathfrak{q}_{1}\right)$ and $\mathfrak{L}\left(\mathfrak{q}_{2}\right)$ are $\operatorname{Int}(\kappa)$-conjugate, then there is $g \in \mathbf{K}_{0}$ such that $\operatorname{Ad}_{\mathrm{K}}(g)\left(\mathfrak{L}\left(\mathfrak{q}_{1}\right)\right)=\mathfrak{L}\left(q_{2}\right)$;

- if $\mathfrak{L}\left(\mathfrak{q}_{1}\right)=\mathfrak{L}\left(\mathfrak{q}_{2}\right)$, then there is an element of the normalizer in $\mathbf{K}_{0}$ of a maximal torus $\mathrm{t}_{0}$ of $\mathfrak{L}\left(\mathfrak{q}_{1}\right) \cap \mathfrak{Q}\left(\mathfrak{q}_{2}\right) \cap \kappa_{0}$ that transforms $\mathfrak{q}_{1}$ into $\mathfrak{q}_{2}$.

The $g$-translation on $N_{\mathrm{q}_{1}}$ realizes a biholomorphism between $N_{\mathrm{q}_{1}}$ and $N_{\mathrm{q}_{2}}$.

If moreover $\mathfrak{q}_{1}, \mathfrak{q}_{2} \in \mathbb{P}_{\kappa}\left(\mathfrak{v}, \mathbf{M}_{0}\right)$, we have a commutative diagram

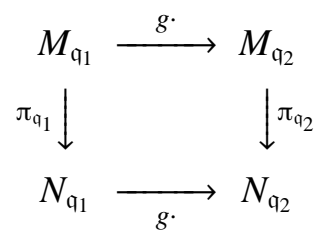

in which $\pi_{\mathrm{q}_{1}}, g \cdot \pi_{\mathrm{q}_{1}}, \pi_{\mathrm{q}_{2}}, g^{-1} \cdot \pi_{\mathrm{q}_{2}}$ are all $C R$-lifts, but in which the left translation on $M$ in the top horizontal line is not $C R$ when $\mathfrak{q}_{1} \neq \mathfrak{q}_{2}$.

Corollary 8.15 (maximal $C R$ structure). If $\mathfrak{q}$ is a minimal element of $\mathbb{P}_{\kappa}\left(\mathfrak{v}, \mathbf{M}_{0}\right)$, then $M_{\mathfrak{q}}$ is maximal among the $\mathbf{K}_{0}$-equivariant strengthening of the $C R$ structure of $M$ for which there is a CR-lift $\phi_{\mathfrak{q}}: M_{\mathfrak{q}} \rightarrow N_{\mathfrak{q}}$ from a complex flag manifold.

Let us use the notation of $\$ 8.1$ In particular, $t_{0}$ is a maximal torus of $\kappa_{0}$ containing a maximal torus $\tau_{0}$ of $\mathrm{m}_{0}$ and $z_{0}=\left\{A \in \mathrm{t}_{0} \mid \operatorname{Ad}_{\kappa}(g)(A)=A, \forall g \in \mathbf{M}_{0}\right\}$. We have the following complement to Proposition 8.11 .

Proposition 8.16. Let $A \in \jmath_{0}$ and $\mathfrak{q}=\mathfrak{q}_{A} \in \mathbb{P}_{\kappa}\left(\mathfrak{v}, \mathbf{M}_{0}\right)$.

A necessary and sufficient condition for having $c_{\mathfrak{q}}=0$ is that $\mathfrak{q}$ is minimal in $\mathbb{P}_{\kappa}\left(\mathfrak{v}, \mathbf{M}_{0}\right)$ and

$$
\mathrm{t}_{0}=\tau_{0}+30 .
$$

A necessary condition in order that there exists $\mathfrak{q} \in \mathbb{P}_{\kappa}\left(\mathfrak{v}, \mathbf{M}_{0}\right) c_{\mathfrak{q}}=0$ is that $\left(\kappa_{0}, \mathrm{~m}\right)$ be of type $\mathrm{II}$.

When this condition is satisfied, and $\mathbf{M}_{0}$ is connected, then $c_{\mathfrak{q}}=0$ for all minimal $\mathfrak{q}_{A} \in \mathbb{P}_{\kappa}\left(\mathfrak{m}, \mathbf{M}_{0}\right)$.

\section{A generalization}

If we drop the assumption that $\left(\kappa_{0}, \mathfrak{v}\right)$ is $\mathfrak{n}$-reductive, only part of Lemma 8.1 remains valid. Namely we obtain: 
Lemma 9.1. Let $\mathfrak{v}$ be any complex Lie algebra and $\mathfrak{q} \in \mathbb{P}_{\kappa}\left(\mathfrak{v}, \mathbf{M}_{0}\right)$. Then $\mathfrak{v}_{\mathfrak{q}}=$ $\mathfrak{v}+\mathfrak{n}_{\kappa}(\mathfrak{q})$ is an $\operatorname{Ad}_{\kappa}\left(\mathbf{M}_{0}\right)$-invariant Lie algebra, with $\mathfrak{n}_{\kappa}\left(\mathfrak{p}_{\mathfrak{q}}\right)=\mathfrak{n}_{\kappa}(\mathfrak{v})+\mathfrak{n}_{\kappa}(\mathfrak{q})$.

Proof. If $\mathfrak{q} \in \mathbb{P}_{\kappa}(\mathfrak{v})$, then $\mathfrak{v} \subset \mathfrak{q}$ and hence $\left[\mathfrak{v}, \mathfrak{n}_{\kappa}(\mathfrak{q})\right] \subset\left[\mathfrak{q}, \mathfrak{n}_{\kappa}(\mathfrak{q})\right] \subset \mathfrak{n}_{\kappa}(\mathfrak{q})$ shows that $\mathfrak{v}_{\mathfrak{q}}=\mathfrak{v}+\mathfrak{n}_{\kappa}(\mathfrak{q})$ is a Lie subalgebra of $\kappa$, containing $\mathfrak{n}_{\kappa}(\mathfrak{q})$ as an ideal of nilpotent elements. It follows that $\operatorname{rad}\left(\mathfrak{v}_{\mathfrak{q}}\right)=\operatorname{rad} \mathfrak{v}+\mathfrak{n}_{\kappa}(\mathfrak{q})$ and hence $\mathfrak{n}_{\kappa}\left(\mathfrak{p}_{\mathfrak{q}}\right)=\mathfrak{n}_{\kappa}(\mathfrak{v})+\mathfrak{n}_{\kappa}(\mathfrak{q})$. Moreover, both $\mathfrak{v}$ and $\mathfrak{n}_{\kappa}(\mathfrak{q})$ are $\operatorname{Ad}_{\kappa}\left(\mathbf{M}_{0}\right)$-invariant, and hence also $\mathfrak{v}_{\mathfrak{q}}$ is $\operatorname{Ad}_{\kappa}\left(\mathbf{M}_{0}\right)$ invariant.

Lemma 9.2. Let $\mathbf{L}_{\mathfrak{q}}$ be the analytic Lie subgroup of $\mathbf{K}_{0}$ with Lie algebra $\mathfrak{v}_{\mathfrak{q}} \cap \kappa_{0}$. Then

$$
\mathbf{M}_{\mathfrak{q}}=\mathbf{M}_{0} \mathbf{L}_{\mathfrak{q}}=\left\{g_{1} g_{2} \mid g_{1} \in \mathbf{M}_{0}, g_{2} \in \mathbf{L}_{\mathfrak{q}}\right\}
$$

is a closed subgroup of $\mathbf{N}_{\mathfrak{q}}$ with $\operatorname{Lie}\left(\mathbf{M}_{\mathfrak{q}}\right)=\mathfrak{v}_{\mathfrak{q}} \cap \kappa_{0}$ and $\mathfrak{v}_{\mathfrak{q}}$ is an $\operatorname{Ad}_{\mathfrak{K}}\left(\mathbf{M}_{\mathfrak{q}}\right)$-invariant subalgebra of $\kappa$.

Proof. Indeed, $\operatorname{ad}\left(\mathbf{M}_{0}\right)\left(\mathbf{L}_{\mathfrak{q}}\right)=\mathbf{L}_{\mathfrak{q}}$ and hence $\mathbf{M}_{q}$ is a compact subgroup of $\mathbf{N}_{\mathrm{q}}$. Since $\mathfrak{m}_{0} \subset \mathfrak{v}_{\mathfrak{q}} \cap \kappa_{0}, \mathbf{M}_{\mathfrak{q}}$ and $\mathbf{L}_{\mathfrak{q}}$ have the same Lie algebra $\mathfrak{v}_{\mathfrak{q}} \cap \kappa_{0}$. Finally, both $\mathbf{M}_{0}$ and $\mathbf{L}_{\mathfrak{q}}$ leave $\mathfrak{v}_{\mathfrak{q}}$ invariant, and thus the same is true for their product group $\mathbf{M}_{\mathfrak{q}}$.

Thus we obtain

Proposition 9.3. For every $\mathfrak{q} \in \mathbb{P}_{\kappa}\left(\mathfrak{v}, \mathbf{M}_{0}\right)$ there is a $\mathbf{K}_{0}$-homogeneous $C R$ manifold $M_{\mathfrak{q}}$, with isotropy $\mathbf{M}_{\mathfrak{q}}$ and CR algebra $\left(\kappa_{0}, \mathfrak{v}_{\mathfrak{q}}\right)$, and we have a commutative diagram of $\mathbf{K}_{0}$-equivariant maps

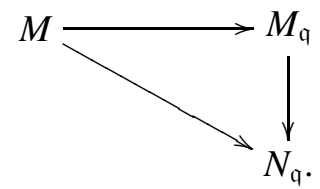

in which the right vertical arrow is a CR-submersion onto a complex flag manifold.

\section{Some EXAMPLES}

We shall discuss in this section some examples of $C R$ manifolds which are minimal orbits $M$ of real forms in complex flag manifolds $F=\mathbf{G} / \mathbf{F}$, with $C R$ algebra $\left(\mathfrak{g}_{0}, \mathfrak{f}\right)$ at the base point.

Example 10.1. Our first example is one in which there is an adapted pair $\left(\theta, \mathfrak{h}_{0}\right)$ with a maximally compact Cartan subalgebra $\mathfrak{h}_{0}$ of $\mathfrak{g}_{0}$, and therefore $M$ is a compact n-reductive compact manifold of type $\mathrm{I}$.

Consider the real form $\mathfrak{g}_{0}=\mathfrak{s l}_{2}(\mathbb{H})$ of $\mathfrak{g}=\mathfrak{s l}_{4}(\mathbb{C})$ and take the minimal orbit $M$ corresponding to the cross marked Satake diagram

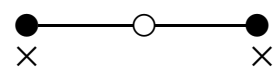

The maximal compact subalgebra of $\mathfrak{s l}_{2}(\mathbb{H})$ is $\mathfrak{s p}_{2}$, with complexification $\kappa=$ $\mathfrak{s p}_{2}(\mathbb{C})$. We give below a matrix description of $\kappa$ and $\mathfrak{v}=\kappa \cap \mathfrak{f}$. Here and in the following we shall consistently use the convention of labelling by $z_{j}$ the entries of the matrices which correspond to the directions in $T^{0,1} M, \zeta_{j} \sim \bar{z}_{j}$ for their conjugate, and by $w_{j}, \bar{w}_{j} \sim \eta_{j}, i t_{j}$ the complex, or imaginary entries corresponding to 
directions transversal to $H M$. We have

$$
\kappa:\left(\begin{array}{cccc}
\lambda_{1} & \zeta_{1} & \eta & \zeta_{2} \\
z_{1} & -\lambda_{1} & z_{2} & -w \\
w & \zeta_{2} & \lambda_{2} & \zeta_{3} \\
z_{2} & -\eta & z_{3} & -\lambda_{2}
\end{array}\right), \quad \mathfrak{v}=\kappa \cap \mathfrak{f}:\left(\begin{array}{cccc}
\lambda_{1} & \zeta_{1} & 0 & \zeta_{2} \\
0 & -\lambda_{1} & 0 & 0 \\
0 & \zeta_{2} & \lambda_{2} & \zeta_{3} \\
0 & 0 & 0 & -\lambda_{2}
\end{array}\right) .
$$

If we denote by $\mathcal{K}=\left\{ \pm e_{1}, \pm e_{2}, \pm e_{1} \pm e_{2}\right\}$ the root system of $\kappa$ with respect to its Cartan subalgebra

$$
\mathrm{t}:\left(\begin{array}{llll}
\lambda_{1} & & & \\
& -\lambda_{1} & & \\
& & \lambda_{2} & \\
& & & -\lambda_{2}
\end{array}\right)
$$

Then $\mathfrak{v}$ is regular, described by

$$
\mathfrak{v}=\kappa \cap \tilde{f}=\mathrm{t} \oplus \sum_{\alpha \in \mathcal{V}_{n}} \kappa^{\alpha}, \quad \text { with } \mathcal{V}_{n}=\left\{2 e_{1}, 2 e_{2}, e_{1}+e_{2}\right\} .
$$

The set $\mathcal{V}_{n}$ is the horocyclic set corresponding to the parabolic set

$$
Q=Q_{n} \cup Q_{r}, \text { with } Q_{n}=\mathcal{V}_{n} \text { and } Q_{r}=\left\{ \pm\left(e_{1}-e_{2}\right)\right\}
$$

which defines the parabolic subalgebra

$$
\mathfrak{q}=\mathrm{t} \oplus \sum_{\alpha \in Q} \kappa^{\alpha} .
$$

Correspondingly, the basis $N_{\mathfrak{q}}$ of the fibration $M \stackrel{\pi}{\longrightarrow} N_{\mathfrak{q}}$ corresponding to the $C R$ submersion $\left(\kappa_{0}, \mathfrak{v}\right) \rightarrow\left(\kappa_{0}, \mathfrak{q}\right)$ is the complex flag manifold defined by the cross marked Satake diagram

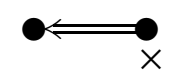

This parabolic $\mathfrak{q}$ is the parabolic regularization of $\mathfrak{p}$. Indeed we have

$$
\mathfrak{n}_{\kappa}(\mathfrak{v}):\left(\begin{array}{cccc}
0 & \zeta_{1} & 0 & \zeta_{2} \\
0 & 0 & 0 & 0 \\
0 & \zeta_{2} & 0 & \zeta_{3} \\
0 & 0 & 0 & 0
\end{array}\right) \quad \mathfrak{v}_{1}=N_{\kappa}\left(\mathfrak{n}_{\kappa}(\mathfrak{v})\right):\left(\begin{array}{cccc}
\lambda_{1} & \zeta_{1} & -\eta & \zeta_{2} \\
0 & -\lambda_{1} & 0 & -w \\
w & \zeta_{2} & \lambda_{2} & \zeta_{3} \\
0 & \eta & 0 & -\lambda_{2}
\end{array}\right)=\mathfrak{v}_{2}=\mathfrak{q},
$$

the latest being the parabolic subalgebra corresponding to the Satake diagram above. In this case $n_{\kappa}(\mathfrak{y})=n_{\kappa}\left(\mathfrak{p}_{1}\right)$, so that all terms in the sequence (6.2) are equal.

Note that $\kappa_{0} \cap \mathfrak{q} \simeq \mathfrak{u}(2)$ and thus $N_{\mathfrak{q}}$ is the Hermitian symmetric space $\mathbf{S p}(2) / \mathbf{U}(2)$. The fiber is $\mathbf{S U}(2) / \mathbf{U}(\mathbf{1}) \simeq S^{2}$, realizing $M$ as an $S^{2}$-bundle over $N_{\mathrm{q}}$.

Example 10.2. Our next example is type II.

Consider the real form $\mathfrak{g}_{0}=\mathfrak{s u}_{1,3}$ of $\mathfrak{g}=\mathfrak{s l}_{4}(\mathbb{C})$, take the minimal orbit $M$ corresponding to the cross marked Satake diagram

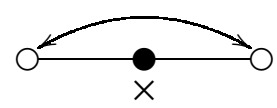

and denote by $\left(\mathfrak{g}_{0}, \mathfrak{f}\right)$ the corresponding $C R$ algebra. We write below the traceless matrix representation of the different Lie algebras involved in the construction of 
the parabolic regularization of $\mathfrak{v}=\kappa \cap \mathfrak{f}$.

$$
\begin{aligned}
& \mathfrak{g}_{0} \simeq \mathfrak{s u}_{1,3}:\left(\begin{array}{cccc}
\lambda & \zeta_{1} & \zeta_{2} & i \sigma \\
-\bar{z}_{1} & i t_{1} & -\bar{z}_{3} & -\bar{\zeta}_{1} \\
z_{2} & z_{3} & i t_{2} & -\bar{\zeta}_{2} \\
i s & z_{1} & -\bar{z}_{2} & -\bar{\lambda}
\end{array}\right), \quad \kappa_{0} \simeq \mathfrak{u}(3):\left(\begin{array}{cccc}
i t_{0} & z_{1} & -\bar{z}_{2} & i s \\
-\bar{z}_{1} & i t_{1} & -\bar{z}_{3} & -\bar{z}_{1} \\
z_{2} & z_{3} & i t_{2} & z_{2} \\
i s & z_{1} & -\bar{z}_{2} & i t_{0}
\end{array}\right) \text {, } \\
& \kappa \simeq \mathbb{C} \ltimes \mathfrak{s l}_{3}(\mathbb{C}):\left(\begin{array}{llll}
\lambda_{0} & z_{1} & \zeta_{2} & w \\
\zeta_{1} & \lambda_{1} & \zeta_{3} & \zeta_{1} \\
z_{2} & z_{3} & \lambda_{2} & z_{2} \\
w & z_{1} & \zeta_{2} & \lambda_{0}
\end{array}\right), \quad \mathfrak{v}=\kappa \cap \mathfrak{f}:\left(\begin{array}{cccc}
\lambda_{0} & 0 & \zeta_{2} & 0 \\
\zeta_{1} & \lambda_{1} & \zeta_{3} & \zeta_{1} \\
0 & 0 & \lambda_{2} & 0 \\
0 & 0 & \zeta_{2} & \lambda_{0}
\end{array}\right), \\
& \mathfrak{n}_{\kappa}(\mathfrak{v}):\left(\begin{array}{cccc}
0 & 0 & \zeta_{2} & 0 \\
\zeta_{1} & 0 & \zeta_{3} & \zeta_{1} \\
0 & 0 & 0 & 0 \\
0 & 0 & \zeta_{2} & 0
\end{array}\right), \quad \mathfrak{v}_{1}:\left(\begin{array}{cccc}
\lambda_{0} & 0 & \zeta_{2} & w \\
\zeta_{1} & \lambda_{1} & \zeta_{3} & \zeta_{1} \\
0 & 0 & \lambda_{2} & 0 \\
w & 0 & \zeta_{2} & \lambda_{0}
\end{array}\right)=\mathfrak{v}_{2}=\mathfrak{q}
\end{aligned}
$$

Then the parabolic q corresponds to the complete complex flag manifold $N_{\mathfrak{q}}$ of $\mathbf{S L}_{3}(\mathbb{C})$, associated to the diagram

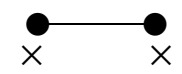

In this case this $\mathfrak{q} \in \mathbb{P}_{\kappa}(\mathfrak{p})$ is also minimal and the fibration $M \stackrel{\pi}{\longrightarrow} N_{\mathfrak{q}}$ associated to the submersion of $C R$ algebras $\left(\kappa_{0}, \mathfrak{v}\right) \rightarrow\left(\kappa_{0}, \mathfrak{v}\right)$ is a circle bundle.

Example 10.3. Consider again the real form $\mathfrak{g}_{0}=\mathfrak{s u}_{1,3}$ of $\mathfrak{g}=\mathfrak{s l}_{4}(\mathbb{C})$ and take this time the minimal orbit $M$ corresponding to the Satake diagram

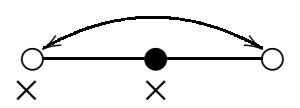

The $\mathbf{G}_{0}$-equivariant $C R$ structure on $M$ defined by the $C R$ algebra $\left(g_{0}, \mathfrak{f}\right)$, in this case, is not maximal (see e.g. [5, Prop.5.9]). We give below the traceless matrix representation of the different Lie algebras involved in the parabolic regularization of $\mathfrak{v}=\kappa \cap \mathfrak{f}:$

$$
\begin{aligned}
& \mathfrak{g}_{0} \simeq \mathfrak{s u}_{1,3}:\left(\begin{array}{cccc}
\lambda & \zeta_{1} & \zeta_{2} & i \sigma \\
w & i t_{1} & -\bar{z}_{1} & -\bar{\zeta}_{1} \\
z_{2} & z_{1} & i t_{2} & -\bar{\zeta}_{2} \\
i s & -\bar{w} & -\bar{z}_{2} & -\bar{\lambda}
\end{array}\right), \quad \kappa_{0} \simeq \mathfrak{u}(3):\left(\begin{array}{cccc}
i t_{0} & -\bar{w} & -\bar{z}_{2} & i s \\
w & i t_{1} & -\bar{z}_{1} & -w \\
z_{2} & z_{1} & i t_{2} & z_{2} \\
i s & \bar{w} & -\bar{z}_{2} & i t_{0}
\end{array}\right), \\
& \kappa \simeq \mathbb{C} \ltimes \mathfrak{s l}_{3}(\mathbb{C}):\left(\begin{array}{cccc}
\lambda_{0} & \eta & \zeta_{2} & \mu \\
w & \lambda_{1} & \zeta_{1} & -w \\
z_{2} & z_{1} & \lambda_{2} & z_{2} \\
\mu & \eta & \zeta_{2} & \lambda_{0}
\end{array}\right), \quad \mathfrak{v}=\kappa \cap \mathfrak{f}:\left(\begin{array}{cccc}
\lambda_{0} & 0 & \zeta_{2} & 0 \\
0 & \lambda_{1} & \zeta_{1} & 0 \\
0 & 0 & \lambda_{2} & 0 \\
0 & 0 & \zeta_{2} & \lambda_{0}
\end{array}\right), \\
& \mathfrak{n}_{\kappa}(\mathfrak{v}):\left(\begin{array}{cccc}
0 & 0 & \zeta_{2} & 0 \\
0 & 0 & \zeta_{1} & 0 \\
0 & 0 & 0 & 0 \\
0 & 0 & \zeta_{2} & 0
\end{array}\right), \quad \mathfrak{v}_{1}:\left(\begin{array}{cccc}
\lambda_{0} & \eta & \zeta_{2} & \mu \\
0 & \lambda_{1} & \zeta_{1} & 0 \\
0 & 0 & \lambda_{2} & 0 \\
\mu & \eta & \zeta_{2} & \lambda_{0}
\end{array}\right)=\mathfrak{v}_{2}=\mathfrak{q}
\end{aligned}
$$

Again, the parabolic $\mathfrak{q}$ is minimal in $\mathbb{P}_{\kappa}(\mathfrak{v})$ and corresponds to the complete complex flag manifold of $\mathbf{S L}_{3}(\mathbb{C})$, associated to the diagram

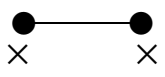


The $C R$ algebra $\left(\kappa_{0}, \mathfrak{v}_{\mathfrak{q}}\right)$, with $\mathfrak{v}_{\mathfrak{q}}=\mathfrak{n}_{\kappa}(\mathfrak{q})+\mathfrak{v} \cap \overline{\mathfrak{v}}$, defines a $C R$ manifold $M_{\mathfrak{q}}$ that is obtained from $M$ by a $\mathbf{K}_{0}$-equivariant strengthening of the $C R$ structure. The corresponding $C R$ fibration $M_{\mathfrak{q}} \stackrel{\pi_{\mathfrak{q}}}{\longrightarrow} N_{\mathfrak{q}}$ is a circle bundle. Note that the $C R$ structure of $M_{\mathfrak{q}}$ is not $\mathbf{G}_{0}$-equivariant and thus it is not equivalent to the $C R$ structure of the minimal orbit of Example10.2

Denote by $M_{1}, M_{2}$ the $C R$ manifolds $M$ and $M_{\mathfrak{q}}$ of Examples 10.210.3 respectively. They correspond to the datum of two different $C R$ structures on the same underlying smooth manifold. Let us describe geometrically their projections into the complete complex flag manifold $N_{\mathfrak{q}}$ of $\mathbf{S} \mathbf{L}_{3}(\mathbb{C})$.

Fix a Hermitian symmetric form $\sigma$ in $\mathbb{C}^{4}$ of signature $(1,3)$ and two $\sigma$-orthogonal subspaces $V_{1}, V_{3}$ on which $\sigma$ is definite. From the $C R$ manifold $M_{1}$ of $\sigma$-isotropic two-planes $L_{2}$ of $\mathbb{C}^{4}$ the $C R$-submersion $M_{1} \rightarrow N_{\mathrm{q}}$ is defined by

$$
L_{2} \longrightarrow L_{2} \cap V_{3} \subset\left(V_{1}+L_{2}\right) \cap V_{3} \subset V_{3},
$$

while the $C R$-submersion $M_{2} \rightarrow N_{\mathfrak{q}}$ from the $C R$ manifold $M_{2}$ of $L_{1} \subset L_{2}$ with a $\sigma$-isotropic line $L_{1}$ and a $\sigma$-isotropic 2-plane $L_{2}$ containing $L_{1}$, is given by

$$
L_{1} \subset L_{2} \longrightarrow\left(L_{1}+V_{1}\right) \cap V_{3} \subset\left(L_{2}+V_{1}\right) \cap V_{3} \subset V_{3} .
$$

Example 10.4. We consider again Example 5.7, We have

$$
\begin{aligned}
& \mathfrak{v}=\kappa \cap \mathfrak{f}:\left(\begin{array}{ccccc}
\lambda_{2} & \zeta_{1} & 0 & 0 & 0 \\
0 & \lambda_{1} & 0 & 0 & 0 \\
0 & \zeta_{2} & \lambda_{0} & \zeta_{2} & 0 \\
0 & 0 & 0 & \lambda_{1} & 0 \\
0 & 0 & 0 & \zeta_{1} & \lambda_{2}
\end{array}\right), \quad \mathfrak{n}_{\mathfrak{g}}(\mathfrak{v}):\left(\begin{array}{ccccc}
0 & \zeta_{1} & 0 & 0 & 0 \\
0 & 0 & 0 & 0 & 0 \\
0 & \zeta_{2} & 0 & \zeta_{2} & 0 \\
0 & 0 & 0 & 0 & 0 \\
0 & 0 & 0 & \zeta_{1} & 0
\end{array}\right) \\
& \mathfrak{v}_{1}:\left(\begin{array}{ccccc}
\lambda_{2} & \zeta_{1} & 0 & \eta_{2} & \mu \\
0 & \lambda_{1} & 0 & \mu & 0 \\
w_{1} & \zeta_{2} & \lambda_{0} & \zeta_{2} & w_{1} \\
0 & \mu & 0 & \lambda_{1} & 0 \\
\mu & \eta_{2} & 0 & \zeta_{1} & \lambda_{2}
\end{array}\right), \quad \mathfrak{n}_{\mathfrak{g}}\left(\mathfrak{v}_{1}\right):\left(\begin{array}{ccccc}
0 & \zeta_{1} & 0 & \eta_{2} & 0 \\
0 & 0 & 0 & 0 & 0 \\
w_{1} & \zeta_{2} & 0 & \zeta_{2} & w_{1} \\
0 & 0 & 0 & 0 & 0 \\
0 & \eta_{2} & 0 & \zeta_{1} & 0
\end{array}\right) \\
& \mathfrak{v}_{2}:\left(\begin{array}{ccccc}
\lambda_{2} & \zeta_{1} & 0 & \eta_{2} & \mu_{2} \\
0 & \lambda_{1} & 0 & \mu_{1} & 0 \\
w_{1} & \zeta_{2} & \lambda_{0} & \zeta_{2} & w_{1} \\
0 & \mu_{1} & 0 & \lambda_{1} & 0 \\
\mu_{2} & \eta_{2} & 0 & \zeta_{1} & \lambda_{2}
\end{array}\right), \quad \mathfrak{n}_{\mathfrak{g}}\left(\mathfrak{p}_{2}\right)=\mathfrak{n}_{\mathfrak{g}}\left(\mathfrak{p}_{1}\right) \Longrightarrow \mathfrak{v}_{2}=\mathfrak{v}_{3}=\mathfrak{q} .
\end{aligned}
$$

Therefore the parabolic regularization yields a $C R$ map $M_{3}^{2,6} \rightarrow N$ of $M_{3}$ onto a compact complex manifold $N_{\mathfrak{q}}=N_{\mathfrak{q}}^{4,0}$ of complex dimension 4 .

The manifold $N_{\mathrm{q}}$ is the complete flag of $\mathbf{S}(\mathbf{U}(2) \times \mathbf{U}(3))$. The fibration can be described explicitly by fixing in $\mathbb{C}^{5}$ a Hermitian symmetric form $\sigma$ of signature $(2,3)$ and two $\sigma$-orthogonal subspaces $V_{2}, V_{3}$ on which the form is definite. We associate to the flag $\left(\ell_{1}, \ell_{3}\right) \in M_{3}$ the point $\left(\ell_{1}+V_{3}\right) \cap V_{2}$ of $\mathbb{P}\left(V_{2}\right) \simeq \mathbb{C P}^{1}$ and the flag $\left(\ell_{3} \cap V_{3},\left(l_{3} \cap V_{3}+\left(l_{1}+V_{2}\right) \cap V_{3}\right)\right) \in\left(\mathfrak{5 r}_{1,2}\left(V_{3}\right)\right.$. The manifold $N_{\mathrm{q}}$ is described by the cross marked Satake diagram

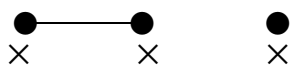


We note that in this case the corresponding $\mathbf{S}(\mathbf{U}(2) \times \mathbf{U}(3))$-equivariant $C R$ map $\pi: M \rightarrow N_{\mathrm{q}}$ is a smooth submersion, and a $C R$-deployment, but not a $C R$ submersion. The space $E_{q} N_{\mathfrak{q}}=\sum_{p \in \pi^{-1}(q)} d \pi\left(T_{p}^{0,1} M\right)$ is a hypersurface in $T_{q}^{0,1} N_{\mathfrak{q}}$ for all $q \in N_{\mathrm{q}}$ and a non formally integrable complex distribution. This example explains the importance of considering commutators in Definition 2.4.

\section{REFERENCES}

1. D. V. Alekseevsky and A. Spiro, Compact homogeneous CR manifolds, J. Geom. Anal. 12 (2002), no. 2, 183-201.

2. Invariant CR structures on compact homogeneous manifolds, Hokkaido Math. J. 32 (2003), no. 2, 209-276.

3. A. Altomani, C. Medori, and M. Nacinovich, The CR structure of minimal orbits in complex flag manifolds, J. Lie Theory 16 (2006), no. 3, 483-530.

4. no. 2, 221-265.

5. 9 (2010), no. 1, 69-109.

6. A. Andreotti and G. A. Fredricks, Embeddability of real analytic Cauchy-Riemann manifolds, Ann. Scuola Norm. Sup. Pisa Cl. Sci. (4) 6 (1979), no. 2, 285-304.

7. A. Borel and J. Tits, Éléments unipotents et sous-groupes paraboliques de groupes réductifs. I, Invent. Math. 12 (1971), 95-104.

8. N. Bourbaki, Éléments de mathématique. Fasc. XXXVIII: Groupes et algèbres de Lie. Chapitre VII: Sous-algèbres de Cartan, éléments réguliers. Chapitre VIII: Algèbres de Lie semi-simples déployées, Actualités Scientifiques et Industrielles, No. 1364, Hermann, Paris, 1975.

9. J.-Y. Charbonnel and H. O. Khalgui, Classification des structures CR invariantes pour les groupes de Lie compacts, J. Lie Theory 14 (2004), no. 1, 165-198.

10. A. A. George Michael, On the conjugacy theorem of Cartain subalgebras, Hiroshima Math. J. 32 (2002), no. 2, 155-163.

11. B. Gilligan and A. T. Huckleberry, Fibrations and globalizations of compact homogeneous CRmanifolds, Izv. Ross. Akad. Nauk Ser. Mat. 73 (2009), no. 3, 67-126.

12. J.E. Humphreys, Linear algebraic groups, Springer-Verlag, New York, 1975, Graduate Texts in Mathematics, No. 21.

13. A. W. Knapp, Lie groups beyond an introduction, second ed., Progress in Mathematics, vol. 140, Birkhäuser Boston Inc., Boston, MA, 2002.

14. B. Kostant, Root systems for Levi factors and Borel-de Siebenthal theory, Symmetry and spaces, Progr. Math., vol. 278, Birkhäuser Boston Inc., Boston, MA, 2010, pp. 129-152.

15. T. Matsuki, Closure relations for orbits on affine symmetric spaces under the action of parabolic subgroups. Intersections of associated orbits, Hiroshima Math. J. 18 (1988), no. 1, 59-67.

16. Y. Matsushima, Espaces homogènes de Stein des groupes de Lie complexes, Nagoya Math. J 16 (1960), 205-218.

17. Y. Matsushima and A. Morimoto, Sur certains espaces fibrés holomorphes sur une variété de Stein, Bull. Soc. Math. France 88 (1960), 137-155.

18. C. Medori and M. Nacinovich, Algebras of infinitesimal CR automorphisms, J. Algebra 287 (2005), no. 1, 234-274.

19. V. V. Morozov, Proof of the theorem of regularity, Uspekhi Mat. Nauk (N.S.) 11 (1956), no. 5(71), 191-194.

20. G. D. Mostow, On covariant fiberings of Klein spaces, Amer. J. Math. 77 (1955), 247-278.

21. __ Covariant fiberings of Klein spaces. II, Amer. J. Math. 84 (1962), 466-474.

22. A. L. Onishchik and È. B. Vinberg, Lie groups and algebraic groups, Springer Series in Soviet Mathematics, Springer-Verlag, Berlin, 1990.

23. V. P. Platonov, A proof of the finiteness hypothesis for solvable subgroups of algebraic groups, Sibirsk. Mat. Ž. 10 (1969), 1084-1090.

24. B. Yu. Weisfeiler, A class of unipotent sub-groups of semi-simple algebraic groups, Uspekhi Mat. Nauk 21 (1966), 222-223.

25. J. A. Wolf, The action of a real semisimple group on a complex flag manifold. I. Orbit structure and holomorphic arc components, Bull. Amer. Math. Soc. 75 (1969), 1121-1237. 
A. Altomani: University of Luxembourg, Campus Kirchberg Mathematics Research Unit, BLG, 6, rue Richard Coudenhove-Kalergi L-1359 Luxembourg Grand-Duchy of Luxembourg E-mail address: andrea.altomani@uni.lu

C. Medori: Dipartimento di Matematica, Università di Parma, Viale G.P. Usberti, 53/A, 43100 Parma (Italy)

E-mail address: costantino.medori@unipr.it

M. Nacinovich: Dipartimento di Matematica, II Università di Roma “Tor Vergata”, Via della Ricerca Scientifica, 00133 Roma (Italy)

E-mail address: nacinovi@mat. uniroma2 .it 